\title{
New insights to the photometric structure of Blue Compact Dwarf galaxies from deep Near-Infrared studies
}

\section{Observations, surface photometry and decomposition of surface brightness profiles}

\author{
K. G. Noeske, P. Papaderos, L. M. Cairós, and K. J. Fricke \\ Universitäts-Sternwarte, Geismarlandstraße 11, 37083 Göttingen, Germany
}

Received 8 October 2002 / Accepted 22 July 2003

\begin{abstract}
We have analyzed deep Near Infrared (NIR) broad band images for a sample of Blue Compact Dwarf Galaxies (BCDs), observed with the ESO NTT ${ }^{\star}$ and Calar Alto ${ }^{\star \star} 3.6 \mathrm{~m}$ telescopes. The data presented here allows for the detection and quantitative study of the extended stellar low-surface brightness (LSB) host galaxy in all sample BCDs. NIR surface brightness profiles (SBPs) of the LSB host galaxies agree at large galactocentric radii with those from optical studies, showing also an exponential intensity decrease and compatible scale lengths. At small to intermediate radii (within 1-3 exponential scale lengths), however, the NIR data reveals for more than one half of our sample BCDs evidence for a significant flattening of the exponential profile of the LSB component. Such profiles (type V SBPs, Binggeli \& Cameron 1991) have rarely been detected in the LSB component of BCDs at optical wavelengths, where the relative flux contribution of the starburst, being stronger than in the NIR, can readily hide a possible central intensity depression in the underlying LSB host. The structural properties, frequency and physical origin of type V LSB profiles in BCDs and dwarf galaxies in general have not yet been subject to systematic studies. Nevertheless, the occurrence of such profiles in an appreciable fraction of BCDs would impose important new observational constraints to the radial mass distribution of the stellar LSB component, as well as to the photometric fading of these systems after the termination of star-forming activities. We test the suitability of two empirical fitting functions, a modified exponential distribution (Papaderos et al. 1996a) and the Sérsic law, for the systematization of the structural properties of BCD host galaxies which show a type $\mathrm{V}$ intensity distribution. Either function has been found to satisfactorily fit a type $\mathrm{V}$ distribution. However, it is argued that the practical applicability of Sérsic fits to the LSB emission of BCDs is limited by the extreme sensitivity of the achieved solutions to, e.g., small uncertainties in the sky subtraction and SBP derivation. We find that most of the sample BCDs show in their stellar LSB host galaxy optical-NIR colors indicative of an evolved stellar population with subsolar metallicity. Unsharp-masked NIR maps reveal numerous morphological details and indicate in some cases, in combination with optical data, appreciable non-uniform dust absorption on a spatial scale as large as $\sim 1 \mathrm{kpc}$.
\end{abstract}

Key words. galaxies: dwarf - galaxies: evolution - galaxies: structure - galaxies: starburst

\section{Introduction}

The star-formation history and chemodynamic evolution of Blue Compact Dwarf (BCD) galaxies are central issues in the contemporary dwarf galaxy research. In spite of being old in their vast majority, BCDs resemble in many aspects unevolved low-mass galaxies in the early Universe. They are gasrich (H I mass fraction of typically $>30 \%$ ) and metal-deficient $\left(Z_{\odot} / 50 \lesssim Z \lesssim Z_{\odot} / 3\right)$ extragalactic systems, undergoing intense, spatially extended star-forming (SF) activity. Such properties

Send offprint requests to: K. G. Noeske,

e-mail: knoeske@uni-sw.gwdg.de

^ European Southern Observatory, program ID 65.N-0318(A).

$\star \star$ German-Spanish Astronomical Center, Calar Alto, operated by the Max-Planck-Institute for Astronomy, Heidelberg, jointly with the Spanish National Commission for Astronomy. are believed to have been common among young low-mass objects at high to intermediate redshift, such as pre-galactic building blocks (Lowenthal et al. 1997; Hirashita et al. 2000; Fujita et al. 2001) or the progenitors of the present-day dwarf spheroidals (e.g. Babul \& Rees 1992; Guzmán et al. 1998). BCDs are therefore convenient nearby laboratories to study at high spatial resolution the impact of collective star formation on the spectrophotometric and chemodynamic properties of these distant and faint extragalactic sources. Moreover, they are important testbeds for deducing constraints to cosmological parameters, such as the primordial ${ }^{4} \mathrm{He}$ abundance ratio, and to monitor the synthesis and dispersal of heavy elements in a nearly pristine environment (Peimbert \& Torres-Peimbert 1974; Pagel et al. 1992; Izotov et al. 1997).

The understanding of the origin and implications of the starburst phenomenon in BCDs is necessary for elucidating 
evolutionary pathways of dwarf galaxies (DGs) in general. Are BCDs active phases in the lifetime of dormant dwarf irregulars (dIs) and do the latter fade to dwarf ellipticals (dEs) once their gas reservoir has been depleted (see e.g. Lin \& Faber 1983; Thuan 1985; Silk et al. 1987; Davies \& Phillipps 1988)? What is the role of the environment (e.g., Babul \& Rees 1992; Pustil'nik et al. 2001) and of Dark Matter (DM; Dekel \& Silk 1986; Ferrara \& Tolstoy 2000), and does the latter invariably dominate the mass within the Holmberg radius of a BCD (Papaderos et al. 1996b; hereafter P96b)? Do most $\mathrm{BCDs}$ undergo intermittent bursts or rather prolonged periods of elevated star formation (Vallenari \& Bomans 1996; Noguchi 2001; Rieschick \& Hensler 2001; Schulte-Ladbeck et al. 2001)? Despite much previous effort, the observational evidence available thus far is still too fragmentary to allow for unambiguous answers to the aforementioned questions.

Recent studies suggest, however, that key information for assessing DG evolution can be inferred from studies of the stellar low-surface brightness (LSB) host galaxy of these systems. In BCDs, the LSB component, underlying the SF regions, has first been disclosed through deep CCD imaging by Loose \& Thuan (1986, hereafter LT86), and has in the following been confirmed and further studied by various authors (cf., e.g., the list given later in this section). This extended stellar host was found to account for $\sim 1 / 2$ of the light inside the 25 B mag/ $\square^{\prime \prime}$ isophote (P96b, Salzer \& Norton 1999), and to typically dominate the intensity and color distribution of BCDs for $\mu \gtrsim 24.5 B \mathrm{mag} / \square^{\prime \prime}$. Such an evenly distributed, evolved stellar component is observed in all types of DGs, except for the extremely rare type of i0 BCDs in the classification scheme of LT86. Its red colors and smooth appearance in the main class of $\mathrm{iE} / \mathrm{nE}$ BCDs (LT86) indicate that these systems are several Gyr old, gas-rich DGs, having not been forming stars at the presently large rate throughout their lifetime. Different lines of evidence, outlined in the following, suggest that elaborate studies of the structural and kinematic properties of the LSB component are fundamental to assess at least two central issues of DG research: the evolutionary connections between DGs and the regulation of the SF process in these systems.

According to the standard evolutionary hypothesis for dwarf galaxies, dIs, dEs and BCDs differ basically by their gas content and the amplitude of their ongoing SF activity (Thuan 1985; Davies \& Phillipps 1988). One would therefore expect that, on average, the evolved stellar LSB host in all these three main DG classes is indistinguishable from one another with respect to its structural properties. However, P96b and subsequent authors (Patterson \& Thuan 1996; Marlowe et al. 1997; Salzer \& Norton 1999, see also Papaderos et al. 2002) found that, at equal absolute magnitude, the stellar LSB component of $\mathrm{iE} / \mathrm{nE} \mathrm{BCD}$ is systematically more compact than other types of DGs. P96b interpreted this structural disparity as the result of adiabatic contraction of the stellar LSB component of $\mathrm{BCDs}$ in response to a large-scale gas inflow, preceding the ignition of a starburst in a BCD. Quite interestingly, subsequent interferometric $\mathrm{HI}$ studies have shown that the gaseous halo of BCDs is by at least a factor of two more centrally concentrated than in dIs (van Zee et al. 1998, 2001; see also
Simpson et al. 2000), lending circumstantial support to the latter hypothesis. Whether or not such observational constraints can be accounted for by the dynamical evolution of the stellar and gaseous matter in DGs (P96b) or, alternatively, an extraordinarily dense Dark Matter halo, being particular to BCD galaxies (Meurer et al. 1998) awaits to be investigated by numerical 3D-simulations. These are also needed to explore a possible connection between the LSB morphology and the evolutionary state of BCDs, as proposed by Noeske et al. (2000).

The evolved LSB component contains, due to its high $M / L$, the bulk of the stellar matter in a BCD. Thus, provided that DM does not dominate within the optical radius, it forms, together with the gaseous component, the gravitational potential within which SF activity occurs. It is therefore worth exploring whether the structural properties of the LSB component influence the SF process in a BCD. P96b found a trend for the fractional surface area of the SF component of BCDs to decrease with increasing LSB luminosity. For a constant $M / L$, this trend translates into a mass-morphology connection for BCDs: SF activity in more massive BCDs occurs mainly in the inner part of the LSB component, leading to a nE morphology and surface brightness profiles (SBPs) sometimes superficially resembling a de Vaucouleurs law. Conversely, SF activity in low-mass BCDs is spread over a larger portion of the LSB host, resulting in an iE morphology and SBPs possessing a conspicuous plateau feature (cf. Papaderos et al. 1996a, hereafter P96a) at intermediate intensity levels.

Our current knowledge of the nature of the underlying LSB component relies mainly on optical surface photometry studies of its faint periphery (e.g. LT86, Kunth et al. 1988, P96a, Telles et al. 1997; Marlowe et al. 1997; Doublier et al. 1997, 1999; Salzer \& Norton 1999; Vennik et al. 2000; Cairós et al. 2001a,b; Makarova et al. 2002). In optical wavelengths, extended stellar and gaseous starburst emission overshines the LSB component out to a galactocentric radius of typically $\sim 2$ exponential scale lengths. Only at larger radii, where the starburst emission is in most cases negligible, the LSB host can be studied directly. However, to explore its possible dynamical influence on the global SF process, it is essential to pin down its intensity distribution at smaller radii, if possible just beneath the SF regions. Deprojection of SBPs would then allow one to put constraints on the density profile and the gravitational potential of the evolved stellar host (cf., e.g., P96a). In addition, one would be able to correct optical colors inside the SF component for the line-of-sight contribution of the underlying stellar background (cf. Cairós et al. 2002; Papaderos et al. 2002), thus better constrain the SF history of these systems.

One way to alleviate the problem of the extended starburst emission is to extend studies to the Near Infrared (NIR). At these wavelengths, the young stellar populations and the ionized gas contribute a smaller fraction of the total light of the galaxy than in the optical. For instance, evolutionary synthesis models by Krüger et al. (1995) predict that a moderately strong burst during its peak luminosity accounts for only $\sim 20 \%$ of a BCD's emission in the $K$, but for $\gtrsim 80 \%$ in the $B$ band. Observations of BCDs also show that starburst emission is weaker in the NIR, and becomes negligible at a smaller galactocentric distance than in the optical (e.g. Vanzi et al. 1996, 2002; 
Beck et al. 1997; Alton et al. 1994; James 1994). NIR data allow therefore to study the stellar LSB component at smaller radii and, using optical-NIR colors (e.g. $B-J$ ), better constrain its formation history. To achieve these objectives one needs to unambiguously detect the LSB component and study its intensity over a sufficient $\operatorname{span}(\Delta \mu \gtrsim 2 \mathrm{mag})$. Empirical estimates, based on published optical and NIR data, suggest that these requirements are met by extending NIR studies to $\sim 22-24 J \mathrm{mag} / \square^{\prime \prime}$. So far, only a few BCDs have been studied at those surface brightness levels with an accuracy high enough to pinpoint structural properties and colors (Mkn 86, Gil de Paz et al. 2000a; Tol 0645-376; Doublier et al. 2001; Tol3; Vanzi et al. 2002).

The present analysis is the first part of an observational project (see also Cairós et al. 2003; hereafter C03), aiming at a systematic study of the NIR properties of nearby BCDs with large array detectors on $4 \mathrm{~m}$-class telescopes. For this purpose, we take advantage of a large set of imaging data, homogeneous with respect to its limiting surface brightness and the methods used in its processing. The observations have been conducted so as to permit NIR surface photometry out to a comparable radius as in optical wavelengths, with the purpose of a multiwavelength investigation of the LSB component.

This paper is structured as follows: In Sect. 2, we describe the sample selection and data acquisition, and discuss the data reduction, photometric transformations and extinction corrections. Section 3 focusses on the derivation of SBPs and their decomposition into the luminosity components owing to the old LSB host and the younger stellar populations and SF regions. In Sect. 4, individual objects are presented in detail. Our results are discussed in Sect. 5. Section 6 summarizes this work.

\section{Observations and data reduction}

\subsection{Sample selection}

Our sample covers the whole morphological spectrum of BCDs, containing both examples of the main morphological class (iE/nE systems according to LT86), and of the less frequent iI/iI,C/i0 BCD classes. The latter subset includes the metal-poor galaxies Tol 65 and Tol 1214-277 $\left(Z<Z_{\odot} / 20\right)$. The sample also includes one intrinsically luminous blue compact galaxy (BCG), UM 448.

Table 1 lists the adopted distance to each BCD. Whenever available, literature distances based on standard candles were preferred for nearby objects. Those literature distances that rely on redshifts have been corrected for peculiar motions within the local supercluster and assume a Hubble constant $H_{0}=$ $75 \mathrm{~km} \mathrm{~s}^{-1} \mathrm{Mpc}^{-1}$. When no literature data was available, the distance was inferred from the $H_{0}$ and the heliocentric velocity listed in the NASA Extragalactic Database (NED). The latter was first transformed to the velocity relative to the local group (LG) centroid using the NED velocity calculator, and subsequently corrected for a LG infall towards the Virgo Cluster center $\left(l=284^{\circ}, b=74^{\circ}\right)$ at $200 \mathrm{~km} \mathrm{~s}^{-1}$ (Tammann \& Sandage 1985).

\subsection{Data acquisition}

The NIR images were observed with the ESO $3.6 \mathrm{~m}$ NTT telescope, La Silla/Chile, during three consecutive nights from April 21st to 24th, 2000. The seeing ranged from 0." 4 to 1 .'0 FWHM and the transparency variations were $\lesssim 1 \%$ during the first and third night, and $\lesssim 2 \%$ during the second night. Additional NIR images were taken at the $3.6 \mathrm{~m}$ telescope of the German-Spanish Astronomical Center, Calar Alto, Spain, during several observing runs. The seeing conditions were generally average (December 26th, 1999: FWHM 1."3-3".5, transparency fair; May 10th-15th, 2000: FWHM 0.'8-1". 4, transparency good to average; October 6th-10th, 2000: FWHM 1'.2-2'. 0, transparency good to fair). Both cameras used, the SOFI at the NTT Nasmyth focus and the OMEGA PRIME at the Calar Alto $3.6 \mathrm{~m}$ telescope's prime focus, were equipped with $1024 \times 1024$ pixel Rockwell HAWAII detectors. The pixel scales for SOFI, using the large field objective, and OMEGA PRIME were 0.'292 and 0.'396, yielding a FOV of 4.94 and 6.76, respectively. The data was taken through the $J$ and $H$ broad band filters, as well as the modified $K$ filters $K_{\mathrm{s}}$ at the NTT and $K^{\prime}$ at Calar Alto, both selected to extenuate the contribution of thermal background.

To achieve an adequate sampling of the background variations, the telescope was offset between exposures typically each $60 \mathrm{~s}$. While compact galaxies were jittered within the FOV, larger objects required to alternately observe the object and the sky. To avoid detector saturation, images were obtained by summing up subexposures of few seconds each. The total on-object exposure times, after rejection of subexposures affected by unstable readout electronics or strong background gradients, are listed in Table 1.

\subsection{Data reduction}

The exposures have been processed semi-interactively, employing our NIR image reduction software based on ESO MIDAS $^{1}$. The procedures used for basic reduction, background subtraction, image alignment and coaddition follow the recipes which are detailed in, e.g., the SOFI User Manual ${ }^{2}$.

Since surface photometry in the LSB regime depends sensitively on the quality of the background subtraction, care has been exercised to eliminate residuals of this correction on both, small and large spatial scales. For this purpose we have implemented a number of additional corrective steps into our software package. For each single science frame of an exposure sequence we first computed an individual master background image, using subexposures that were taken closest in time, but at a sufficiently large distance from the target. Prior to the calculation of the master background frame, the input background images were cleaned from bright contaminating sources and normalized to the same mean intensity (see the SOFI User Manual for details). The master background frame computed this way was in turn scaled to the background level of the respective science frame, and subtracted. Each of the

\footnotetext{
1 Munich Image data Analysis System, provided by the European Southern Observatory (ESO).

2 Issue 1.2, available online at http://www . eso.org
} 
Table 1. Sample galaxies.

\begin{tabular}{|c|c|c|c|c|c|c|c|c|c|}
\hline Object & $\begin{array}{l}\text { RA(J2000) } \\
\operatorname{Dec}(\mathrm{J} 2000) \\
\text { (2) }\end{array}$ & $\begin{array}{c}t_{J, \mathrm{NTT}} \\
t_{J, \mathrm{CA}} \\
{[\mathrm{s}]} \\
(3)\end{array}$ & $\begin{array}{c}t_{H, \mathrm{NTT}} \\
t_{H, \mathrm{CA}} \\
{[\mathrm{s}]} \\
(4)\end{array}$ & $\begin{array}{c}t_{K_{\mathrm{S}}, \mathrm{NTT}} \\
\mathrm{t}_{K^{\prime}, \mathrm{CA}} \\
{[\mathrm{s}]} \\
(5)\end{array}$ & $\begin{array}{c}M_{B} \\
\text { (ref.) } \\
{[\mathrm{mag}]} \\
(6)\end{array}$ & $\begin{array}{c}A_{B} \\
{[\mathrm{mag}]} \\
(7)\end{array}$ & $\begin{array}{c}D \\
\text { (ref.) } \\
{[\mathrm{Mpc}]} \\
(8)\end{array}$ & $\begin{array}{c}F W H M \\
\text { (final)` } \\
{\left[{ }^{\prime \prime}\right]} \\
(9)\end{array}$ & $\begin{array}{l}\text { Other } \\
\text { names }\end{array}$ \\
\hline $\begin{array}{l}\text { Tol } 3 \\
\text { (iE) }\end{array}$ & $\begin{array}{r}10^{\mathrm{h}} 06^{\mathrm{m}} 33^{\mathrm{s}} .6 \\
-29^{\circ} 56^{\prime} 09^{\prime} .0\end{array}$ & $\begin{array}{c}1440 \\
-\end{array}$ & $\begin{array}{c}1560 \\
-\end{array}$ & $\begin{array}{c}2100 \\
-\end{array}$ & $\begin{array}{l}-18.0 \\
\text { (a) }\end{array}$ & 0.33 & $\begin{array}{l}13.8 \\
\text { (a) }\end{array}$ & 1.2 & $\begin{array}{l}\text { NGC 3125; ESO 435-G041; } \\
\text { Tol1004-296 }\end{array}$ \\
\hline $\begin{array}{l}\text { Haro } 14 \\
(\mathrm{iE} / \mathrm{nE})\end{array}$ & $\begin{array}{r}00^{\mathrm{h}} 45^{\mathrm{m}} 46^{\mathrm{s}} 4 \\
-15^{\circ} 35^{\prime} 499^{\prime} 0\end{array}$ & $\begin{array}{c}- \\
1440\end{array}$ & $\begin{array}{c}- \\
1680\end{array}$ & $\begin{array}{c}- \\
1920\end{array}$ & $\begin{array}{l}-17.0 \\
\text { (a) }\end{array}$ & 0.09 & $\begin{array}{l}12.5 \\
\text { (a) }\end{array}$ & 1.6 & $\begin{array}{l}\text { NGC 0244; UGCA 010; } \\
\text { VV } 728\end{array}$ \\
\hline $\begin{array}{l}\text { UM } 461 \\
\text { (iI) }\end{array}$ & $\begin{array}{r}11^{\mathrm{h}} 51^{\mathrm{m}} 33^{\mathrm{s}} .0 \\
-02^{\circ} 22^{\prime} 23^{\prime \prime} .0\end{array}$ & $\begin{array}{c}1500 \\
-\end{array}$ & $\begin{array}{c}1500 \\
-\end{array}$ & $\begin{array}{c}2100 \\
-\end{array}$ & $\begin{array}{l}-14.9 \\
\text { (j) }\end{array}$ & 0.08 & $\begin{array}{l}14.3 \\
\text { (e) }\end{array}$ & 0.7 & SCHG 1148-020 \\
\hline $\begin{array}{l}\text { He } 2-10 \\
(\mathrm{iE} / \mathrm{nE})\end{array}$ & $\begin{array}{r}08^{\mathrm{h}} 36^{\mathrm{m}} 15^{\mathrm{s}} .0 \\
-26^{\circ} 24^{\prime} 34^{\prime \prime} .0\end{array}$ & $\begin{array}{c}1140 \\
-\end{array}$ & $\begin{array}{c}1500 \\
-\end{array}$ & $\begin{array}{c}2040 \\
-\end{array}$ & $\begin{array}{c}-18.7 \\
(\mathrm{k})\end{array}$ & 0.48 & $\begin{array}{l}8.7 \\
\text { (f) }\end{array}$ & 0.9 & ESO 495-G021 \\
\hline $\begin{array}{l}\text { Tol 1400-411 } \\
\text { (iI,C) }\end{array}$ & $\begin{array}{r}14^{\mathrm{h}} 03^{\mathrm{m}} 21^{\mathrm{s}} .0 \\
-41^{\circ} 22^{\prime} 44^{\prime \prime} .0\end{array}$ & $\begin{array}{c}1140 \\
-\end{array}$ & $\begin{array}{c}1500 \\
-\end{array}$ & $\begin{array}{c}2100 \\
-\end{array}$ & $\begin{array}{c}-16.5 \\
(1)\end{array}$ & 0.30 & $\begin{array}{l}4.8 \\
\text { (c) }\end{array}$ & 0.9 & $\begin{array}{l}\text { NGC 5408; Tol 116; } \\
\text { ESO 325-G047 }\end{array}$ \\
\hline $\begin{array}{l}\text { Pox 4/Pox 4B } \\
\text { (iI + comp.) }\end{array}$ & $\begin{array}{r}11^{\mathrm{h}} 51^{\mathrm{m}} 11^{\mathrm{s}} .6 \\
-20^{\circ} 36^{\prime} 02^{\prime \prime} .0\end{array}$ & $\begin{array}{c}1500 \\
-\end{array}$ & $\begin{array}{c}1500 \\
-\end{array}$ & $\begin{array}{c}2100 \\
-\end{array}$ & $\begin{array}{c}-18.8 \\
(\mathrm{~m})\end{array}$ & 0.17 & $\begin{array}{l}46.7 \\
\text { (b) }\end{array}$ & 0.6 & CAM 1148-2020 \\
\hline $\begin{array}{l}\text { Tol 65 } \\
\text { (i0/iI,C) }\end{array}$ & $\begin{array}{c}12^{\mathrm{h}} 25^{\mathrm{m}} 46^{\mathrm{s}} .9 \\
-36^{\circ} 14^{\prime} 01^{\prime \prime} 0 \mathrm{~s}\end{array}$ & $\begin{array}{c}2100 \\
-\end{array}$ & $\begin{array}{c}2100 \\
-\end{array}$ & $\begin{array}{c}3000 \\
-\end{array}$ & $\begin{array}{c}-15.3 \\
(\mathrm{n})\end{array}$ & 0.32 & $\begin{array}{l}34.2 \\
\text { (b) }\end{array}$ & 0.6 & $\begin{array}{l}\text { ESO 380-G027; } \\
\text { Tol 1223-359 }\end{array}$ \\
\hline $\begin{array}{l}\text { Tol 1214-277 } \\
\text { (i0/il,C) }\end{array}$ & $\begin{array}{r}12^{\mathrm{h}} 17^{\mathrm{m}} 17^{\mathrm{s}} .1 \\
-28^{\circ} 02^{\prime} 33^{\prime \prime} .0\end{array}$ & $\begin{array}{c}1200 \\
-\end{array}$ & $\begin{array}{c}1500 \\
-\end{array}$ & $\begin{array}{c}2100 \\
-\end{array}$ & $\begin{array}{l}-16.9 \\
\text { (o) }\end{array}$ & 0.28 & $\begin{array}{l}102.6 \\
\text { (b) }\end{array}$ & 0.9 & $\begin{array}{l}\text { SCHG 1214-277; } \\
\text { Tol } 21\end{array}$ \\
\hline $\begin{array}{l}\text { Mkn } 178 \\
\text { (iE) }\end{array}$ & $\begin{array}{r}11^{\mathrm{h}} 33^{\mathrm{m}} 29^{\mathrm{s}} .1 \\
+49^{\circ} 14^{\prime} 17^{\prime \prime} .0\end{array}$ & $\begin{array}{c}240 \\
-\end{array}$ & $\begin{array}{c}1380 \\
-\end{array}$ & $\begin{array}{c}1380 \\
-\end{array}$ & $\begin{array}{c}-13.9 \\
(\mathrm{p})\end{array}$ & 0.08 & $\begin{array}{l}4.2 \\
(\mathrm{~h})\end{array}$ & 1.6 & $\begin{array}{l}\text { UGC 06541; HOLMBERG 263A; } \\
\text { CGCG 242-046; SBS 1130+495 }\end{array}$ \\
\hline $\begin{array}{l}\text { Mkn } 1329 \\
\text { (iI,C) }\end{array}$ & $\begin{array}{r}12^{\mathrm{h}} 37^{\mathrm{m}} 03^{\mathrm{s}} .0 \\
+06^{\circ} 55^{\prime} 36^{\prime \prime} .0\end{array}$ & $\begin{array}{c}300 \\
1200\end{array}$ & $\begin{array}{l}360 \\
360\end{array}$ & $\begin{array}{l}480 \\
960\end{array}$ & $\begin{array}{c}-16.8 \\
\text { (i) }\end{array}$ & 0.10 & $\begin{array}{l}16.0 \\
\text { (i) }\end{array}$ & 0.7 & $\begin{array}{l}\text { VCC 1699; IC 3589/91; } \\
\text { UGC } 7790\end{array}$ \\
\hline $\begin{array}{l}\mathrm{IC} 4662 \\
(\mathrm{dI} / \mathrm{iE})\end{array}$ & $\begin{array}{r}17^{\mathrm{h}} 47^{\mathrm{m}} 06^{\mathrm{s}} .4 \\
-64^{\circ} 38^{\prime} 25^{\prime \prime} \cdot 0\end{array}$ & $\begin{array}{c}1980 \\
-\end{array}$ & $\begin{array}{c}1620 \\
-\end{array}$ & $\begin{array}{c}660 \\
-\end{array}$ & $\begin{array}{c}-14.9 \\
(1)\end{array}$ & 0.30 & $\begin{array}{l}2.0 \\
(\mathrm{~g})\end{array}$ & 0.8 & $\begin{array}{l}\text { ESO 102-G014; } \\
\text { He 2-269 }\end{array}$ \\
\hline $\begin{array}{l}\mathrm{UM} 448 \\
\text { (iI/BCG) }\end{array}$ & $\begin{array}{r}11^{\mathrm{h}} 42^{\mathrm{m}} 12^{\mathrm{s}} .4 \\
+00^{\circ} 20^{\prime} 03^{\prime \prime} .0\end{array}$ & $\begin{array}{l}300 \\
840\end{array}$ & $\begin{array}{l}360 \\
900\end{array}$ & $\begin{array}{c}480 \\
1200\end{array}$ & $\begin{array}{l}-19.8 \\
(1)\end{array}$ & 0.11 & $\begin{array}{l}76.1 \\
(\mathrm{~d})\end{array}$ & 0.7 & $\begin{array}{l}\text { UGC 06665; SCHG 1139+006; } \\
\text { Ark 312; ARP 161; Mkn } 1304\end{array}$ \\
\hline
\end{tabular}

a: Resolution of the best image set available for the respective galaxy, after reduction and combination (a): Marlowe et al. (1999), (b): inferred from the heliocentric velocity $v_{\text {hel }}$ listed in the NED, corrected for solar motion with respect to the center of the Virgo Cluster and adopting $H_{0}=$ $75 \mathrm{~km} \mathrm{~s}^{-1} \mathrm{Mpc}^{-1}$ (cf. Sect. 2.1). (c): Karachentsev et al. (2002), (d): Mirabel \& Sanders (1988), (e): $v_{\mathrm{LG}}$ from Smoker et al. (2000), corrected for Local Group infall to the Virgo Cluster like b); (f): Tully (1988), (g): Heydari-Malayeri 1990, (h): Schulte-Ladbeck et al. (2000), (i): Yasuda et al. (1997), (j): $m_{B}$ from Méndez \& Esteban (2000), (k): $m_{B}$ from Papaderos (1998), (1): $m_{B}$ from the RC3 (de Vaucouleurs et al. 1991), (m): $m_{B}$ for Pox 4 from Méndez \& Esteban (1999); no published $m_{B}$ for Pox 4B (n): $m_{B}$ from P99, (o): $m_{B}$ from F01, (p): $m_{B}$ from Papaderos et al. (2002).

resulting subexposures was subsequently checked for a possible residual background gradient and, whenever necessary, rectified using a first order polynomial fit. The result image was obtained through combination of all background-corrected and coregistered subexposures. In this procedure, out of all pixels with identical sky coordinates, only those at sound detector coordinates have been included in the calculations.

Small residual background variations in the final images needed to be interactively corrected by fitting twodimensional polynomials. Alternatively, regions still affected by smallscale residuals in the background subtraction were extracted as subimages, corrected in the latter manner, and inserted back into the original frame. Likewise, bleeding artifacts, caused by bright sources in the NTT/SOFI FOV, or blooming of bright, overexposed foreground stars in the very outskirts of IC 4662 (northern edge) and Tol 1400-411 (east and west) were modeled and subtracted out. SBPs of the latter two galaxies were only analyzed well above intensity levels where slight residuals from the replaced bright stars could possibly contribute.
Final images taken in the same filter during different nights or at different telescopes were aligned to each other, transformed to equal pixel scales and resolutions, and coadded after weighting each one by its $(S / N)^{2}$. The $F W H M$ of the result images is listed in Table 1. Images used for aperture or surface photometry were manually cleaned for fore- and background sources.

\subsection{Flux calibration}

The SOFI data was calibrated by observing at different airmasses standard stars from Persson et al. (1998) six times each night. The excellent photometric stability throughout the NTT observing run has allowed for the derivation of an airmassdependent calibration with a scatter of $\lesssim 0.01$ mag during nights 1 and 3 and $\$ 0.02$ mag during night 2 . Zero points and airmass-dependent calibration coefficients both agree well with the average values supplied by the NTT/SOFI support team. Images of UM 448 and Mkn 1329, obtained by 
combining Calar Alto and NTT data, were calibrated using aperture photometry of bright stars in the SOFI FOV. Despite different $K$ filters used at those telescopes, the integral $K_{\mathrm{s}}$ fluxes are essentially preserved, as color terms in the filter transformations (Eqs. (3)) are not exceeding a few $0.01 \mathrm{mag}$.

The BCDs Haro 14 and Mkn 178, for which no NTT observations are available, were calibrated using the Two Micron All Sky Survey (2MASS) catalogue ${ }^{3}$ (Cutri et al. 2000; Jarrett et al. 2000). As 2MASS data (cf. Andreon 2002) may, due to their limited sensitivity, slightly underestimate the flux within the extended LSB component, we computed calibration terms using 2MASS field stars in the close vicinity of Haro 14 and Mkn 178.

\subsubsection{Transformation to other NIR photometric systems}

Unless stated otherwise, all magnitudes and colors given in this paper refer to the calibrations described in the previous section, and to the photometric systems defined by the instrumental setup of each telescope (see Sect. 2). However, whenever photometric quantities of the sample BCDs are compared either among each other, or with model predictions and data from the literature, they are first transformed to the 2MASS photometric system, using the relations described below.

The calibration obtained at the NTT with the SOFI instrument is based on standards by Persson et al. (1998), and can therefore be transformed to the Persson et al. (1998) Las Campanas Observatory (LCO) system using the color transformations given by the SOFI user manual (Issue 1.3, $16 / 08 / 2000)$. A transformation from the latter system to the 2MASS system is described by Carpenter (2001). Combination of both latter transformations yields the relations:

$K_{\mathrm{s}, 2 \mathrm{M}}=K_{\mathrm{s}, \mathrm{S}}+0.021(J-K)-0.010$

$(J-H)_{2 \mathrm{M}}=0.995(J-H)_{\mathrm{S}}+0.015(J-K)+0.002$

$\left(H-K_{\mathrm{S}}\right)_{2 \mathrm{M}}=1.029\left(H-K_{\mathrm{S}}\right)_{\mathrm{S}}-0.046(J-K)+0.005$,

where the indices "2M" and "S" denote the 2MASS and SOFI systems, respectively. Cumulative uncertainties introduced by this transformation are $\$ 0.01 \mathrm{mag}$ for $K_{\mathrm{s}}$ and $\lessgtr 0.015 \mathrm{mag}$ for $(J-H)$ and $\left(H-K_{\mathrm{s}}\right)$, given the typical range of values expected for NIR colors of BCDs.

No transformations to other standard NIR systems are available for the photometric system defined by the Calar Alto $3.6 \mathrm{~m}$ telescope and the OMEGA PRIME camera. As stated in Sect. 2.4, the calibration of the Calar Alto frames is tied to the 2MASS zero points. The remaining uncertainties introduced by the unknown color terms can be estimated from Carpenter (2001) to be

$\mid(\text { color })_{2 \mathrm{M}}-(\text { color })_{\Omega^{\prime}}|\lesssim| 0.08(\text { color })_{\Omega^{\prime}} \mid$

per transformed color, i.e., the approximate upper limit of the color terms entering into the transformation between standard NIR photometric systems.

\footnotetext{
${ }^{3}$ http://www.ipac.caltech.edu/2mass/
}

The $K^{\prime}$ and $K_{\mathrm{s}}$ magnitudes are related by the following equations (cf. Wainscoat \& Cowie 1992 and the SOFI user manual):

$K_{\mathrm{s}}=K+0.005(J-K)$

$K=K^{\prime}-(0.22 \pm 0.03)(H-K)$.

\subsubsection{Extinction correction}

Magnitudes and colors given in this paper are corrected for Galactic extinction, adopting values derived from the $B$ band extinction maps by Schlegel et al. (1998) (cf. Table 1) and the standard $\left(R_{V}=3.1\right)$ extinction law (Cardelli et al. 1989) implemented into the NED. No attempt was made to correct for internal extinction, since this is known to vary spatially even in the most metal-deficient BCDs (cf., e.g., Guseva et al. 2001; Cannon et al. 2002; Hunt et al. 2003) and can be reliably constrained in the SF regions only.

\section{Data analysis and SBP derivation}

\subsection{Derivation of surface brightness and color profiles}

Surface photometry aims at a standardized one-dimensional representation of a galaxy's two-dimensional flux pattern. One technique to compute surface brightness profiles (SBPs) requires the determination of the size $A(\mu)$ of the galaxy in $\square^{\prime \prime}$ for a series of surface brightness levels $\mu$ (mag/ $\left.\square^{\prime \prime}\right)$. By this definition, the equivalent radius $R^{*}=\sqrt{(A(\mu) / \pi)}$ is a monotonic function of the surface brightness $\mu$ and vice versa. In order to derive SBPs this way, one has to keep track of the morphology and angular extent of a BCD throughout its intensity span, i.e. in general to be able to interpolate an isophote down to the faintest measured level $\mu$ of an SBP. By this condition one can visually check for and screen-out fore- or background sources in the periphery of the galaxy, thus make sure that source confusion does not affect SBPs at faint levels. This task is more difficult to achieve when computing SBPs employing photon statistics inside circular or elliptical annuli, extending out to a user-defined maximal radius $r_{\max }$. SBPs derived for an irregular system by such techniques may vary from case to case, depending on, e.g., the adopted $r_{\max }$ or "center" of the galaxy. It should be borne in mind that techniques of this kind, when applied to galaxies with a morphology significantly departing from the assumed circular symmetry, can strongly overestimate the exponential scale length and underestimate the central intensity of the LSB component (cf., e.g., Marlowe et al. 1997).

In the present analysis, we compute SBPs using method iv), described in Papaderos et al. (2002). This is a hybrid technique, incorporating features of both aforementioned approaches (determination of the $R^{*}$ corresponding to a user-defined $\mu$, as opposed to the determination of the mean surface brightness $\bar{\mu}$ inside a circular annulus with a user-defined radius $R^{*} \pm \Delta R^{*}$ ).

For a set of $n$ intensity intervals $I_{n}$, with a mean intensity decreasing as $n$ increases, masks $M_{n}$ are generated, each of which extracts from a smoothed image of the galaxy the areas with 
intensities within $I_{n}$. The equivalent radius $R_{n}^{\star}$ corresponding to the mask area $A_{n}$ is:

$R_{n}^{\star}=\left(\frac{1}{\pi}\left[\sum_{i=1}^{n-1} A_{i}+\frac{1}{2} A_{n}\right]\right)^{\frac{1}{2}}$.

The surface brightness $\mu\left(R_{n}^{\star}\right)$ is calculated from the mean intensity of the original image within the area $M_{n}$. Method iv) overcomes the problem of the artificial SBP flattening for low $S / N$ levels (see discussion in e.g. Noeske 1999; Noeske et al. 2000 and Cairós 2000) and proves reliable down to very faint surface brightness levels ( $I_{n}<$ sky noise).

As a check for consistency, we also derived SBPs through ellipse fitting to isophotes or methods ii) and iii) in P96a, applying in all cases a minimum of image filtering to moderate photon noise. The resulting SBPs have been compared with deep optical surface photometry to ensure that the underlying LSB component has been detected and modelled (see Sect. 3.2) over a sufficient radius range.

Color profiles were derived by subtracting the SBPs from each other, after the latter had been smoothed to equal $F W H M$. Surface brightness and color profiles, corrected for Galactic extinction (Sect. 2.4.2), are shown in Figs. 2 through 14.

\subsubsection{Notes on the errors}

The uncertainties of each data point include the fully propagated effects of (i) Poisson photon noise and (ii) residual background variations on various spatial scales. The latter originate both from small residuals from the background reduction, and from undetected fore- and background sources. The treatment of (i) is explained in, e.g., P96a or Cairós et al. (2001a). The resulting uncertainties are in most cases small, owing to the large number of pixels typically involved in the calculation of each profile point at small $S / N$ levels, i.e. in the outer LSB region of a galaxy. In these low $S / N$ regions, the uncertainties from (ii) are typically dominant: background variations on spatial scales which are not small compared to the size of the considered mask segment or aperture do not average out.

The amplitude of such variations was estimated as the standard deviation of the mean intensities measured in quadratic apertures, placed on the background well away from the object, with sizes $\lambda$ of the order $1 / 10$ of the object's diameter. The resulting uncertainties of each SBP point might be decreased by a factor of $\sqrt{A_{n} / \lambda^{2}}$, accounting for the averaging of background variations over the area $A_{n}$ (Eq. (4)) of the respective mask segment. This first-order correction was however discarded, since the background variations cannot be determined at the position of the galaxy's LSB component, and since the outer mask segments cut through large areas of the image, possibly sampling individual stronger background anomalies. Error bars shown in the SBPs and color profiles (Figs. 2 through 14) therefore represent upper limits, i.e. typically overestimates, to the true errors, which are difficult to quantify. Nevertheless, the error constraints shown here give a better estimate of the true errors than the, typically too small, pure Poisson noise errors.

The reliability limits of the SBPs were not estimated from these error constraints, but determined at a surface brightness where an isophote could still be robustly interpolated on a mildly smoothed image.

\subsection{Profile decomposition}

The stellar emission of a BCD is due to the superposition of two distinct populations with respect to their $\mathcal{M} / \mathcal{L}$ ratio and spatial extent: (i) the underlying LSB host galaxy which, owing to its high $\mathcal{M} / \mathcal{L}$, contains the bulk of the system's stellar mass, and (ii) the younger stellar population, attributable to the ongoing and recent SF activity. In the main class of $\mathrm{iE} / \mathrm{nE} \mathrm{BCDs}$, the younger stellar component dominates the optical emission inside $\sim 2$ exponential scale lengths of the underlying LSB host and contributes, together with ionized gas emission, $\sim 1 / 2$ of the $B$ light within the $25 B \mathrm{mag} / \square^{\prime \prime}$ isophote (P96b, Noeske 1999; Salzer \& Norton 1999). Evidently, a meaningful study of the structural properties of a BCD requires the decomposition of SBPs into these two main photometric components. In the following, we employ a simple SBP decomposition scheme, in which we fit only the LSB emission. Subtraction of the best fit LSB model from the SBP allows us to deduce the luminosity fraction and spatial extent of the superimposed SF component. In order to make sure that extended starburst emission does not affect the decomposition results, we fitted the LSB component beyond a transition radius $R_{\mathrm{T}}^{*}$ (cf. P96a, C03), where optical and optical-NIR color gradients vanish and isophotes become more regular. In addition, whenever available, we used $\mathrm{H} \alpha$ maps to trace the size of the SF component, and get from it an additional constraint to $R_{\mathrm{T}}^{*}$. The LSB emission has been fit out to the radius where SBPs became increasingly uncertain as a result of background noise (typically for $\lesssim 0.5 \sigma_{\mathrm{bgr}}$ for method iv). The fitted SBP range in $J$ is indicated with the dashed-gray line at the bottom of each profile (Figs. 2-14).

An exponential fitting law

$I\left(R^{\star}\right)=I_{\mathrm{E}, 0} \exp \left(-\frac{R^{\star}}{\alpha}\right)$

with a central intensity $I_{\mathrm{E}, 0}$ and an exponential scale length $\alpha$ has been found to approximate well the intensity distribution of our sample galaxies in their LSB component. An exponential law is also known to fit well the outer parts of dwarf irregulars (dIs, Patterson \& Thuan 1996; van Zee 2000) and dwarf ellipticals (dEs, Vigroux et al. 1988; Binggeli \& Cameron 1991) over a few scale lengths.

However, for several of our sample BCDs, an extrapolation of the exponential LSB slope to smaller radii yields for $R^{*} \sim 1 \ldots 3 \alpha$ a higher intensity than the observed value. Thus, a meaningful decomposition of such SBPs cannot be achieved on the usual assumption that the exponential law is valid in the LSB component all the way to $R^{*}=0^{\prime \prime}$. Instead, one has to adopt an alternative fitting formula, approaching the exponential law for large radii and flattening in its inner part. Such a distribution should be compatible to the "type II" profiles of disc galaxies (Freeman 1970, see also MacArthur et al. 2003), or the "type V" profiles described for spheroidal early type DGs by Binggeli \& Cameron (1991).

Previous optical studies have allowed for the detection and modelling of such an inwards flattening exponential LSB 
profile in a few BCDs only (e.g. I Zw 115, P96a; Tol 65, Papaderos et al. 1999, hereafter P99; Tol 1214-277, Fricke et al. 2001, hereafter F01; SBS 0940+544, Guseva et al. 2001). However, SBPs of this kind do not appear to be rare among intrinsically faint dEs and dIs. They have been observed in several dEs with and without a central nucleus (cf. Binggeli \& Cameron 1991; Cellone et al. 1994; Young \& Currie 1994, 1995), and Vennik et al. (2000) deduce a fraction of $>10 \%$ for the SBPs of late-type dwarf galaxies falling into this category. In fact, a casual inspection of the sample of Patterson \& Thuan (1996), Makarova et al. (1998), Vennik et al. (2000) and van Zee (2000) reveals several examples of dIs showing an exponential outer LSB slope and a pronounced flattening for intermediate to small radii (e.g., UGC 2034, UGC 2053, UGC 5423, UGC 9128, UGC 10669 in the sample of Patterson \& Thuan 1996, KKH35 and KKSG 19 in Makarova et al. 1998, or UGCA 9, UGCA 15, UGC 2345, UGC 9240, UGC 10445 in the sample by van Zee 2000). Other examples are the dIs Holmberg I (Ott et al. 2001), Holmberg II (Noeske 1999), H 1032-2722 (Duc et al. 1999) and Kar 50 (Davidge 2002). Following the nomenclature of Binggeli \& Cameron (1991) we shall henceforth refer to this type of SBPs as to type V.

The physical origin and exact form of type $\mathrm{V}$ profiles in puffed-up stellar systems has not yet been studied in detail, neither observationally nor theoretically. Therefore it is difficult to say which fitting formula approximates best their intensity distribution (see discussion in Sect. 5.3). An empirical fitting function that yields by deprojection a finite luminosity density for $R^{*}=0^{\prime \prime}$ has been proposed in P96a, as

$$
I\left(R^{\star}\right)=I_{\mathrm{E}, 0} \exp \left(-\frac{R^{\star}}{\alpha}\right)\left\{1-q \exp \left(-P_{3}\left(R^{\star}\right)\right)\right\}
$$

with

$P_{3}\left(R^{\star}\right)=\left(\frac{R^{\star}}{b \alpha}\right)^{3}+\left(\frac{R^{\star}}{\alpha} \cdot \frac{1-q}{q}\right)$.

The modified exponential distribution Eq. (6), in the following referred to as med, flattens with respect to a pure exponential law inside of a cutoff radius $b \alpha$, and attains at $R^{*}=0^{\prime \prime}$ an intensity given by the relative depression parameter $q=\Delta I / I_{\mathrm{E}, 0}<1$. An advantage of the med is that its exponential part (left part of Eq. (6)), depending on $I_{\mathrm{E}, 0}$ and $\alpha$ only, can be separately constrained by fitting Eq. (5) to the outer exponential part of a type $\mathrm{V}$ profile. This has been done by means of unweighted fits, after SBPs were splined to equidistant radius steps, in order to ensure that the fit solution is not biased through a clustering of points to a specific radius range. Once the scale length $\alpha$ is fixed, the multiplicative right-hand side part of Eq. (6) is a function of $b$ and $q$, only. Because the $(b, q)$ parameter space has not been explored so far, e.g., by fitting Eq. (6) to a sample of dEs with type V SBPs, it is difficult to judge which values are appropriate for dwarf galaxies falling into this category. P96a inferred a tentative ratio of $b / q \sim 3$, they noted, however, that this could be luminosity-dependent.

In order to deduce plausible constraints to $(b, q)$, we first subtracted from the $J$ image of each sample BCD most of the irregular starburst emission. The latter has been approximated by an unsharp-mask version of the original image (Sect. 3.4), adjusted such that no regular emission from the LSB component was removed. $\mathrm{H} \alpha$ exposures and color maps were used to further ascertain that the subtracted emission was not part of the underlying host galaxy. $J$ band exposures processed this way were then used to compute SBPs for the LSB component. These profiles, denoted $J_{\mathrm{LSB}}$, allow to better trace the LSB component down to smaller $R^{*}$ than the ones derived prior to partial subtraction of the starburst light. By fitting Eq. (6) to the $J_{\mathrm{LSB}}$ SBPs we derived $b, q$ (first column in Table 2). In most cases, fit uncertainties are 0.1 and 0.05 for $b$ and $q$, respectively. No reliable $J_{\text {LSB }}$ profiles could be computed for Tol 1214-277. For this system we fixed $(b, q)$ to values inferred by F01 from optical VLT data.

Alternatively, type $\mathrm{V} J_{\mathrm{LSB}}$ profiles were fit with a Sérsic model (Sérsic 1968) of the form

$$
I\left(R^{\star}\right)=I_{\mathrm{S}, 0} \exp \left(-\frac{R^{\star}}{\beta}\right)^{1 / \eta}
$$

The exponent ${ }^{4} \eta$ in Eq. (8) is a handy indicator of systematic deviations of the observed SBP from an exponential distribution. An $\eta<1$ corresponds to a convex profile, which might be compatible to a med distribution with $q>0$ (see discussion in Sect. 5.3), whereas an $\eta>1$ indicates a concave profile which, in the special case of $\eta=4$, translates into the de Vaucouleurs law (see, e.g., Caon et al. 1993).

Note that Eq. (8) approximates well the projected light of a variety of stellar populations with both a nearly constant $\mathcal{M} / \mathcal{L}$ (ellipticals or bulges; Caon et al. 1993; Andredakis et al. 1995; Graham et al. 1996, early-type dwarfs; Cellone et al. 1994; Young \& Currie 1994) or strongly varying $\mathcal{M} / \mathcal{L}$ (for instance, $\mathrm{nE}$ BCDs or the plateau component in the SBP of iE BCDs; P96a). Therefore a Sérsic exponent $\eta>1$ gives no strong indication for a stellar system being similar to an intrinsically luminous early-type galaxy (see also discussion in C03).

The Sérsic exponents of the LSB components $\left(\eta_{\mathrm{LSB}}\right)$ were obtained from non-weighted fits to the $J_{\text {LSB }}$ SBPs (see above) of type $\mathrm{V}$ profiles. This limits $\eta_{\mathrm{LSB}}$ to values $<1$. We avoided to fit Eq. (8) to the outer part of SBPs alone, i.e. for $R^{*} \geq R_{\mathrm{T}}^{*}$, as solutions obtained this way are very uncertain and depend strongly on the accuracy of the sky subtraction (see Sect. 5.3 and detailed discussion in C03). Notwithstanding the fact that Eqs. (6) and (8) give comparably good fits in terms of $\chi^{2}$, we decided not to include the full $\left(I_{\mathrm{S}, 0}, \beta, \eta\right)$ Sérsic solutions for the $J_{\text {LSB }}$ SBPs in Table 2. Instead, in its Col. 1, we quote only the Sérsic exponent $\eta_{\mathrm{LSB}}$, the uncertainties of which are estimated to be of the order of $20 \%$.

The photometric quantities of the sample BCDs are summarized in Table 2. BCDs without signatures of a type $\mathrm{V}$ profile in their underlying LSB component are marked with an asterisk in Col. 1 . For the remaining systems we list the $(b, q)$ and $\eta_{\mathrm{LSB}}$ parameters, as obtained respectively by fitting Eqs. (6) and (8)

\footnotetext{
4 For consistency with, e.g., Caon et al. (1993) and C03 we define here the Sérsic shape parameter as $\eta$, i.e. the reciprocal exponent in Eq. (8). Note that in other studies (Cellone et al. 1994; Young \& Currie 1994,1995 ; P96a) the Sérsic shape parameter is referred to as $1 / \eta$.
} 
Table 2. Structural properties of the dwarfs ${ }^{a}$; see also the discussion of individual objects.

\begin{tabular}{|c|c|c|c|c|c|c|c|c|c|c|c|c|}
\hline $\begin{array}{l}\text { Name } \\
(b, q)^{b} \\
\eta_{\mathrm{LSB}}^{c} \\
(1)\end{array}$ & Band & $\begin{array}{c}\mu_{E, 0} \\
\mathrm{mag} / \square^{\prime \prime}\end{array}$ & $\begin{array}{c}\alpha \\
\mathrm{kpc}\end{array}$ & $\begin{array}{c}m_{\mathrm{LSB}}^{\text {fit }} \\
\text { mag }\end{array}$ & $\begin{array}{l}P_{\text {iso }} \\
\mathrm{kpc}\end{array}$ & $\begin{array}{r}m_{P_{\text {iso }}} \\
\text { mag }\end{array}$ & $\begin{array}{l}E_{\text {iso }} \\
\mathrm{kpc}\end{array}$ & $\begin{array}{r}m_{\mathrm{E}_{\text {iso }}} \\
\text { mag }\end{array}$ & $\begin{array}{r}m_{\mathrm{SBP}} \\
\mathrm{mag}\end{array}$ & $\begin{array}{l}m_{\mathrm{tot}} \\
\mathrm{mag}\end{array}$ & $\begin{array}{c}r_{\mathrm{eff}}, r_{80} \\
\mathrm{kpc}\end{array}$ & $\eta_{\mathrm{SBP}}$ \\
\hline $\mathrm{Tol} 3^{e}$ & $J$ & $18.24 \pm 0.05$ & $0.52 \pm 0.01$ & 11.78 & 1.20 & 12.42 & 2.29 & 11.86 & 11.31 & 11.29 & $0.61,1.23$ & 1.45 \\
\hline \multirow[t]{2}{*}{$\star$} & $H$ & $17.74 \pm 0.06$ & $0.53 \pm 0.01$ & 11.26 & 1.18 & 11.76 & 2.07 & 11.37 & 10.73 & 10.69 & $0.61,1.22$ & 1.43 \\
\hline & $K_{\mathrm{s}}$ & $17.45 \pm 0.09$ & $0.51 \pm 0.01$ & 11.06 & 1.18 & 11.48 & 2.12 & 11.15 & 10.50 & 10.46 & $0.57,1.15$ & 1.49 \\
\hline Haro $14^{f}$ & $J$ & $17.46 \pm 0.12$ & $0.37 \pm 0.01$ & 12.70 & 1.39 & 12.45 & 1.88 & 12.82 & 11.81 & 11.78 & $0.61,1.21$ & 1.18 \\
\hline $3.6,0.94$ & $H$ & $16.97 \pm 0.26$ & $0.39 \pm 0.02$ & 12.11 & 1.37 & 11.69 & 1.76 & 12.31 & 11.12 & 11.07 & $0.61,1.22$ & 1.25 \\
\hline 0.49 & $K^{\prime}$ & $16.88 \pm 0.94$ & $0.39 \pm 0.08$ & 11.99 & 1.32 & 11.54 & 1.83 & 12.17 & 10.98 & 10.97 & $0.60,1.21$ & 1.45 \\
\hline $\mathrm{UM} 461^{e}$ & $J$ & $19.37 \pm 0.18$ & $0.21 \pm 0.01$ & 15.56 & 0.42 & 16.19 & 0.69 & 15.88 & 15.09 & 15.04 & $0.34,0.57$ & 1.96 \\
\hline $2.3,0.85$ & $H$ & $18.82 \pm 0.25$ & $0.21 \pm 0.02$ & 14.98 & 0.35 & 16.12 & 0.60 & 15.46 & 14.66 & 14.57 & $0.37,0.61$ & 1.82 \\
\hline 0.49 & $K_{\mathrm{s}}$ & $18.47 \pm 0.79$ & $0.19 \pm 0.04$ & 14.79 & 0.36 & 15.75 & 0.63 & 15.14 & 14.46 & 14.37 & $0.32,0.54$ & 2.04 \\
\hline Henize $2-10^{e}$ & $J$ & $18.46 \pm 0.03$ & $0.67 \pm 0.01$ & 10.46 & 0.87 & 10.57 & 2.80 & 10.55 & 9.80 & 9.70 & $0.41,1.26$ & 2.50 \\
\hline \multirow[t]{2}{*}{$\star$} & $H$ & $17.75 \pm 0.04$ & $0.64 \pm 0.01$ & 9.85 & 0.78 & 9.94 & 2.51 & 9.96 & 9.17 & 9.13 & $0.40,1.22$ & 2.44 \\
\hline & $K_{\mathrm{s}}$ & $17.51 \pm 0.03$ & $0.62 \pm 0.01$ & 9.68 & 0.79 & 9.62 & 2.56 & 9.78 & 8.95 & 8.89 & $0.34,1.04$ & 2.44 \\
\hline Tol 1400-411 & $J$ & $18.29 \pm 0.11$ & $0.33 \pm 0.01$ & 11.18 & 0.69 & 12.67 & 1.41 & 11.32 & 10.99 & 10.91 & $0.60,0.98$ & 2.04 \\
\hline $3.0,0.82$ & $H^{d}$ & $17.30 \pm 0.14$ & $0.27 \pm 0.01$ & 10.57 & 0.31 & 13.65 & 1.18 & 10.71 & 10.57 & 10.55 & $0.57,0.93$ & 1.33 \\
\hline 0.63 & $K_{\mathrm{s}}$ & $17.39 \pm 0.09$ & $0.30 \pm 0.01$ & 10.43 & 0.50 & 12.49 & 1.28 & 10.58 & 10.32 & 10.31 & $0.59,0.97$ & 1.75 \\
\hline $\operatorname{Pox} 4^{e}$ & $J$ & $19.26 \pm 0.15$ & $0.86 \pm 0.03$ & 15.15 & 2.27 & 14.80 & 2.87 & 15.54 & 14.24 & 14.20 & $1.24,2.17$ & 1.75 \\
\hline $2.7,0.90$ & $H^{d}$ & $19.10 \pm 0.31$ & $0.88 \pm 0.07$ & 14.95 & 2.18 & 14.35 & 1.79 & 16.10 & 13.85 & 13.81 & $1.27,2.15$ & 1.45 \\
\hline 0.42 & $K_{\mathrm{s}}{ }^{d}$ & - & - & - & - & - & - & - & 13.54 & 13.52 & $1.18,2.09$ & 1.96 \\
\hline Pox $4 \mathrm{~B}^{e}$ & $J$ & $19.47 \pm 0.40$ & $0.29 \pm 0.04$ & 17.37 & 0.51 & 19.29 & 0.93 & 17.67 & 17.21 & 17.12 & $0.54,0.87$ & 0.75 \\
\hline $2.0,0.80$ & $H^{d}$ & - & - & - & - & - & - & - & 16.83 & 16.72 & $0.51,0.80$ & 0.77 \\
\hline 0.49 & $K_{\mathrm{s}}{ }^{d}$ & - & - & - & - & - & - & - & 16.75 & 16.52 & $0.47,0.69$ & 0.75 \\
\hline Tol 65 & $J$ & $19.58 \pm 0.18$ & $0.28 \pm 0.02$ & 17.25 & 0.43 & 17.47 & 0.82 & 17.79 & 16.57 & 16.55 & $0.37,0.80$ & 1.59 \\
\hline $2.7,0.90$ & $H^{d}$ & - & - & - & - & - & - & - & 16.23 & 16.30 & $0.42,0.84$ & - \\
\hline 0.46 & $K_{\mathrm{s}}{ }^{d}$ & - & - & - & - & - & - & - & 16.03 & 15.99 & $0.24,0.61$ & - \\
\hline Tol 1214-277 & $J^{d}$ & $19.16 \pm 0.24$ & $0.53 \pm 0.03$ & 18.05 & 0.75 & 18.56 & 1.69 & 18.55 & 17.46 & 17.41 & $0.86,1.83$ & 1.89 \\
\hline $3.3,0.92$ & $H^{d}$ & - & - & - & - & - & - & - & 17.22 & 17.26 & $1.15,1.90$ & - \\
\hline 0.48 & $K_{\mathrm{s}}{ }^{d}$ & - & - & - & - & - & - & - & 16.94 & 17.07 & $0.81,1.67$ & - \\
\hline Mkn $178^{f}$ & $J$ & $20.62 \pm 0.11$ & $0.28 \pm 0.01$ & 12.94 & 0.39 & 14.36 & 0.62 & 13.41 & 12.78 & 12.70 & $0.34,0.57$ & 1.27 \\
\hline \multirow[t]{2}{*}{$\star$} & $H^{d}$ & $19.87 \pm 0.18$ & $0.26 \pm 0.02$ & 12.35 & 0.26 & 14.85 & 0.51 & 12.92 & 12.29 & 12.25 & $0.34,0.58$ & 1.12 \\
\hline & $K^{\prime d}$ & $19.92 \pm 0.43$ & $0.27 \pm 0.04$ & 12.31 & 0.36 & 14.00 & 0.52 & 12.91 & 12.13 & 12.01 & $0.36,0.58$ & 0.97 \\
\hline $\mathrm{Mkn} 1329^{e}$ & $J$ & $19.25 \pm 0.04$ & $0.67 \pm 0.01$ & 12.81 & 0.99 & 14.61 & 2.32 & 13.01 & 12.64 & 12.60 & $1.12,1.97$ & 1.01 \\
\hline $1.6,0.70$ & $H^{d}$ & $18.81 \pm 0.01$ & $0.71 \pm 0.01$ & 12.25 & 1.01 & 13.85 & 2.09 & 12.57 & 12.00 & 11.94 & $1.17,2.08$ & 1.11 \\
\hline 0.68 & $K_{\mathrm{s}}$ & $18.24 \pm 0.06$ & $0.60 \pm 0.01$ & 12.03 & 0.79 & 14.56 & 2.09 & 12.23 & 11.95 & 11.93 & $1.09,1.87$ & 0.72 \\
\hline $\mathrm{IC} 4662^{e}$ & $J$ & $16.79 \pm 0.24$ & $0.15 \pm 0.01$ & 10.60 & 0.55 & 10.32 & 0.83 & 10.73 & 9.68 & 9.64 & $0.29,0.53$ & 1.41 \\
\hline $4.8,0.97$ & $H$ & $16.61 \pm 0.73$ & $0.15 \pm 0.02$ & 10.36 & 0.58 & 9.64 & 0.69 & 10.66 & 9.15 & 9.05 & $0.29,0.52$ & 1.37 \\
\hline 0.48 & $K_{\mathrm{s}}{ }^{d}$ & - & - & - & - & - & - & - & 9.04 & 8.93 & $0.27,0.48$ & 1.75 \\
\hline $\mathrm{UM} 448^{e}$ & $\begin{array}{l}J \\
\end{array}$ & $20.55 \pm 0.07$ & $3.83 \pm 0.10$ & 13.48 & 4.26 & 12.50 & 8.66 & 13.92 & 12.17 & 12.15 & $1.28,3.63$ & 2.33 \\
\hline \multirow[t]{2}{*}{$\star$} & $H$ & $19.95 \pm 0.17$ & $4.02 \pm 0.31$ & 12.77 & 4.08 & 11.88 & 7.60 & 13.39 & 11.52 & 11.50 & $1.32,3.94$ & 2.40 \\
\hline & $K_{\mathrm{s}}$ & $19.55 \pm 0.15$ & $3.61 \pm 0.19$ & 12.61 & 4.01 & 11.53 & 8.16 & 13.05 & 11.22 & 11.30 & $1.21,3.24$ & 2.44 \\
\hline
\end{tabular}

${ }^{a}$ : All values are corrected for Galactic extinction, adopting the $A_{B}$ from Table 1 (cf. Sect. 2.4.2).

${ }^{b}, c$ : See Sect. 3.2 for details. Objects whose LSB component has been modelled by a pure exponential fit (Eq. (5)) are marked with an asterisk.

${ }^{d}$ : Decomposition uncertainty higher (faint object, or data affected by nearby bright stars).

${ }^{e}$ : Calibration obtained at the ESO NTT, cf. Sect. 2.4.

${ }^{f}$ : Calibrated using field stars from the 2MASS catalogue, cf. Sect. 2.4.

${ }^{g}$ : Fit radius interval and $(b, q)$ adopted from F01.

to $J_{\mathrm{LSB}} \mathrm{SBPs}$. Columns 3 and 4 list, respectively, the extrapolated central surface brightness $\mu_{\mathrm{E}, 0}\left(\mathrm{mag} / \square^{\prime \prime}\right)$ and exponential scale length (kpc), obtained by fitting Eq. (5) to the outer exponential LSB part of each SBP. Column 5 lists the total apparent magnitude of the LSB component, computed by extrapolating the fitted model (i.e. Eq. (5) or Eq. (6)) to $R^{\star}=\infty$. Columns 6 through 9 list the radii and magnitudes of the starforming (P) and underlying stellar LSB component (E), as 
obtained by profile decomposition. Following P96a, we measure the respective radial extent $\left(P_{\text {iso }}, E_{\text {iso }}\right)$ and encircled magnitude $\left(m_{P_{\text {iso }}}, m_{E_{\text {iso }}}\right)$ of each component at an isophotal level iso, taken to be $23 \mathrm{mag} / \square^{\prime \prime}$ for $J$ and $22 \mathrm{mag} / \square^{\prime \prime}$ for $H$ and $K$. The isophotal radii determined for the sample BCDs at $23 \mathrm{~J}$ mag/ $\square^{\prime \prime}$ turn out to be comparable to those obtained from optical SBPs at $25 \mathrm{~B} \mathrm{mag} / \square^{\prime \prime}\left(P_{25}\right.$ and $E_{25}$ in P96a). Column 10 lists the magnitude from an SBP integration out to the last data point, and total magnitudes from aperture measurements (cf. Sect. 3.3) are listed in Col. 11. The radii $r_{\text {eff }}$ and $r_{80}$, enclosing $50 \%$ and $80 \%$ of the SBP's flux are included in Col. 12 . Finally, a formal Sérsic exponent for the whole SBP $\left(\eta_{\mathrm{SBP}}\right)$, for later comparison with literature data, is listed in Col. 13 of Table 2.

Errors of the fitting law parameters determined from unweighted fits are an underestimation of the true errors. LSB slope differences between $J, H$ and $K$ (Table 2) should therefore not be considered significant, but rather to reflect the true exponential slope uncertainties, which are typically up to $10 \%$ in $J$, and may become somewhat larger in $H$ and $K$, depending on the data quality.

\subsection{Aperture photometry and total magnitudes}

Since a SBP can only be accurately derived in regions with a sufficiently high $S / N$ (i.e., in general, down to the minimum intensity level in which the morphology of a BCD can still be visually checked and the problem of source confusion can be handled; cf. Sect. 3.1), profile integration out to the last measured data point may, in some cases, underestimate the object's total flux. For extended LSB sources, or when NIR SBPs do not go sufficiently deep (e.g., the $K_{\mathrm{s}}$ SBP of Tol 1214-277), the fractional flux missed can easily exceed $10 \%$. Similar problems may affect growth curve flux determinations, since these basically rely on a crude SBP derivation and can sensitively depend on the quality of background subtraction.

We therefore measured total magnitudes within polygonal apertures which extend typically out to 1.5 Holmberg radii (Col. 11 of Table 2), after removal of fore- and background sources from the area of interest (cf. Sect. 2.3). Errors take into account the Poisson noise and small-scale variations of the local background.

Magnitudes and colors of selected features, such as stellar clusters or H II regions, have been corrected for the flux contribution of the underlying LSB component, by interpolating the mean surface brightness of adjacent regions. Values computed this way are marked with the superscript $\ddagger$. As pointed out in Cairós et al. (2002) and Papaderos et al. (2002), corrections for the LSB emission are generally not negligible in optical wavelengths. That this statement is also true in the NIR domain is illustrated on the example of the iE BCD UM 461 (Fig. 1); correction for the flux contribution of the LSB background shifts even the brightest $\mathrm{SF}$ region ( $\mathrm{a}, m_{J} \ddagger=17.26 \mathrm{mag}$, open circle) of this system by $+0.25 \mathrm{mag}$ and $-0.1 \mathrm{mag}$ in the $J-H$ and $H-K_{\mathrm{s}}$ color diagram. A census and photometric study of compact stellar clusters in the extended BCD sample included

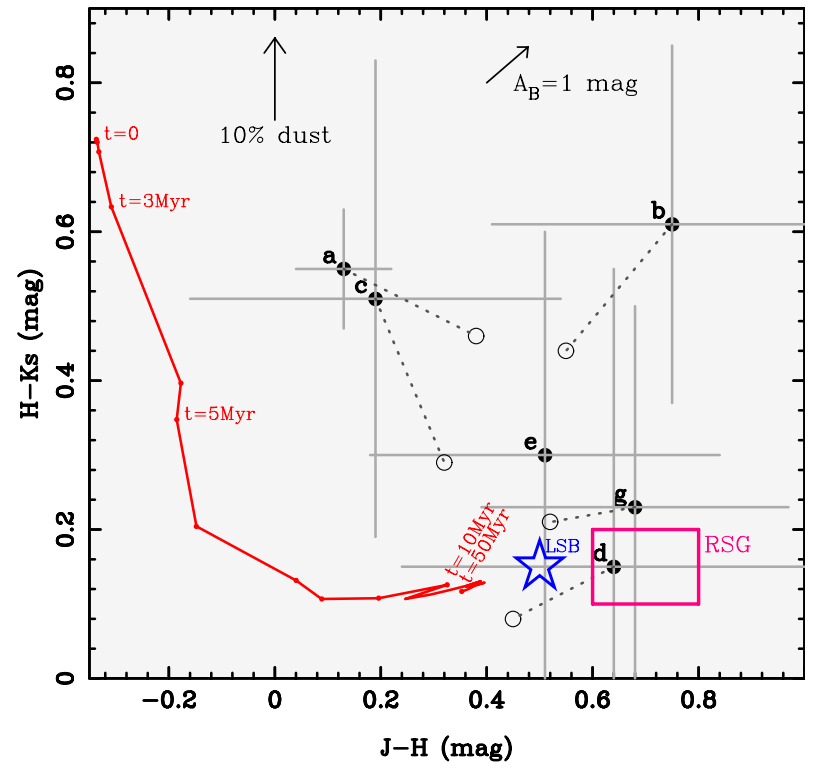

Fig. 1. NIR two-color diagram for the brightest compact regions in the iI BCD UM 461. Filled circles show the colors of the regions a through $\mathrm{g}$ indicated in Fig. 5, after correction for the flux contribution of the underlying LSB host galaxy. Open circles, connected with dotted lines, indicate the color of the respective region prior to that correction. The star marks the color of the stellar host galaxy ("LSB"). The color range covered by red supergiants in the SMC (Elias et al. $1985)$ is shown by the box labelled RSG to the lower right. The temporal evolution of the $J-H$ vs. $H-K_{\mathrm{s}}$ color for an instantaneously formed stellar population with $Z_{\odot} / 10$ for an age between $t=0$ and $t=50 \mathrm{Myr}$ (solid line) has been calculated with the PEGASE code (Fioc \& Rocca-Volmerange 1997). The colors for $t=0$ correspond to purely gaseous emission. Arrows depict the effect of a $10 \%$ contribution of warm $(\sim 200 \mathrm{~K})$ dust to the $K$ band (cf. Campbell \& Terlevich 1984), as well as the extinction vector. All colors are transformed to the 2MASS photometric system.

in this project after correction for the LSB- and ionized gas emission will be presented in a subsequent paper.

\subsection{Unsharp masking}

The considerable intensity range of a BCD, from its faint LSB outskirts to the brightest nuclear starburst region, renders the detection of fine coherent morphological features in the central portion of the galaxy difficult. We use therefore a modified unsharp masking technique (cf., e.g., Papaderos 1998), referred to in the following as hierarchical binning (hb) - transformation. This contrast-enhancing procedure is stable against noise at low intensities and allows for the flux determination of faint sources within the bright background of a BCD, with an angular size smaller than a user-defined value. Morphological features of interest revealed using this procedure are displayed in the grayscale/isophote insets of the sample galaxies, and described in Sect. 4.

\subsection{Colors of the underlying LSB host galaxy}

Colors of the LSB host galaxy were derived as the errorweighted mean of the color profiles for $R^{*}>R_{\mathrm{T}}^{*}$, after 

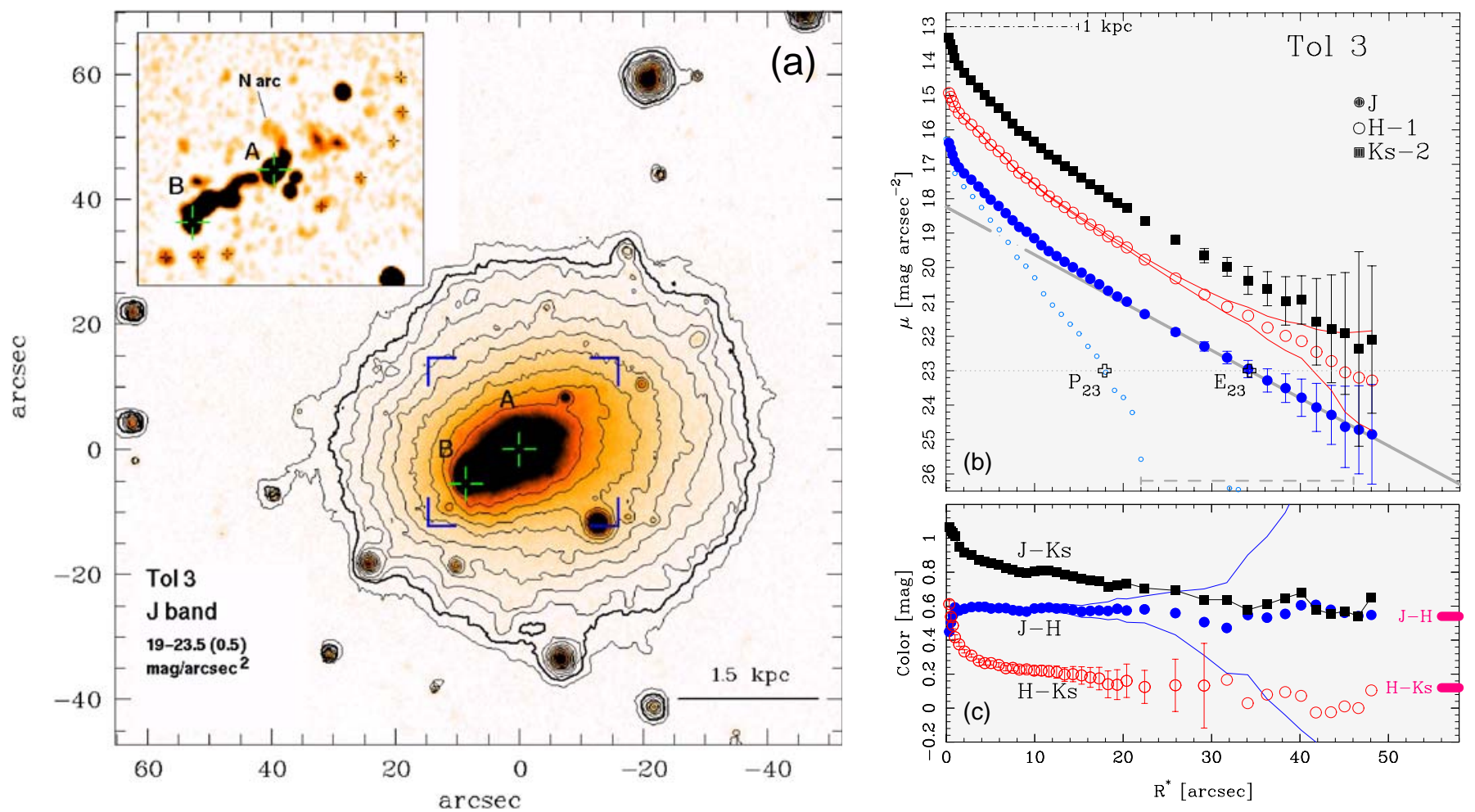

Fig. 2. a) Contours overlaid with a $J$ image of Tol $3(D=13.8 \mathrm{Mpc})$. North is up, east to the left. Contours, corrected for Galactic extinction, go from 19 to $23.5 \mathrm{~J} \mathrm{mag} / \square^{\prime \prime}$ in increments of $0.5 \mathrm{mag}$. The $23 \mathrm{~J} \mathrm{mag} / \square^{\prime \prime}$ isophote is illustrated by the thick contour. The brighter star-forming region A and the fainter knot B (following the denomination by Schaerer et al. 1999) are marked with crosses. The inset shows a contrastenhanced (see Sect. 3.4) and magnified version of the central region of the BCD (indicated by brackets in the contour map image). Compact sources arranged along the southwestern arc-like chain are marked with small crosses. b) Surface brightness profiles (SBPs) of Tol 3 in the $J$, $H$ and $K_{\mathrm{s}}$, corrected for galactic extinction. For a better visualization, the $H$ and $K_{\mathrm{s}}$ SBPs are shifted by -1 and -2 mag, respectively. The thick solid line illustrates an exponential fit to the stellar LSB component in $J$ (cf. Sect. 3.2), computed in the radius range indicated by the light gray, long-dashed bar at the bottom of the figure. The emission in excess to the fit (small open circles) is attributable to the starburst component, which dominates the light in the inner part of Tol 3. The isophotal radii $P_{23}$ and $E_{23}$ of the star-forming and LSB component at the surface brightness level of $23 J \mathrm{mag} / \square^{\prime \prime}$ (horizontal dotted line) are indicated. The bar at the upper left of the figure corresponds to a galactocentric distance of $1 \mathrm{kpc}$. c) Color profiles, computed by subtraction of the SBPs shown in the upper right panel. The thick lines at the rightmost part of the diagram indicate the mean $J-H$ and $H-K_{\mathrm{s}}$ colors of the LSB component (see Sect. 3.5).

rejection of deviant points, being probably affected by uncertainties in the sky determination, and local residuals in the subtraction of background sources. For Tol 1400-411, Pox 4, Mkn 178 and IC 4662, uncertainties in the LSB colors are larger, due to extended starburst emission or crowding with nearby bright stars. As for the very metal-deficient systems Tol 65 and Tol 1214-277, the faintness of their LSB component in $K_{\mathrm{s}}$ has not permitted, despite generous exposure times, to pin down their $H-K_{\mathrm{s}}$ colors.

Whenever calibrated optical data were available, opticalNIR colors were derived. Because the quality of the SBPs was typically better in $J$ than in $H$, and the $J-H$ color shows little evolution with time (few $0.1 \mathrm{mag}$ ) for old ( $\gtrsim 1 \mathrm{Gyr}$ ) stellar populations, we derived $B-J$ colors instead of the more commonly used $B-H$ colors.

The mean colors of the host galaxies are shown at the right edge of each color profile (Figs. 2-14), in the photometric system in which the respective galaxy was observed and calibrated (see Sect. 2.4.1). Table 3 lists the NIR colors, transformed to the 2MASS photometric system to facilitate comparisons and the $B-J$ color, where available. Errors give cumulative uncertainties in the calibration, transformation to the 2MASS system, and the scatter and systematic uncertainty of each color profile.

\section{Results and discussion of individual objects}

\subsection{Tol 3 (NGC 3125)}

This luminous $\left(M_{B} \sim-18.0\right.$; Marlowe et al. 1999) and relatively metal-rich $\left(Z_{\odot} / 6 \ldots Z_{\odot} / 3\right.$; Kobulnicky et al. 1999; Schaerer et al. 1999; Marlowe et al. 1999 and references therein) BCD is known to be a member of the NGC 3175 galaxy group (García 1993). NIR and optical images reveal two compact $(\$ 0.3 \mathrm{kpc})$ high-surface brightness (HSB) regions: the brighter northwestern knot A, roughly coinciding with the geometrical center of the smooth LSB host galaxy, and the fainter knot B, located $\sim 1 \mathrm{kpc}$ southeast of A (see Fig. 2a). Either knot is the locus of ongoing star formation, as witnessed by the detection of Wolf-Rayet features (Kunth \& Sargent 1981; Vacca \& Conti 1992; Schaerer et al. 1999) and red supergiants from CO absorption studies (Campbell \& Terlevich 1984). 
Table 3. Colors of the host galaxy ${ }^{a}$.

\begin{tabular}{lccc}
\hline \hline Object & $\begin{array}{c}J-H \\
{[\mathrm{mag}]}\end{array}$ & $\begin{array}{c}H-K_{\mathrm{s}} \\
{[\mathrm{mag}]}\end{array}$ & $\begin{array}{c}B-J \\
{[\mathrm{mag}]}\end{array}$ \\
\hline Tol 3 & $0.55 \pm 0.07$ & $0.10 \pm 0.06$ & - \\
${\text { Tol } 65^{c}}_{\text {Tol 1214-277c }}$ & $0.48 \pm 0.17^{b}$ & - & $1.3 \pm 0.15^{b}$ \\
Tol 1400-411 & $0.39 \pm 0.16^{b}$ & - & $1.0 \pm 0.13^{b}$ \\
Pox 4 & $0.43 \pm 0.08$ & $0.26 \pm 0.09$ & $1.45 \pm 0.16$ \\
Pox 4B & $0.42 \pm 0.13$ & $0.12 \pm 0.13$ & - \\
UM 448 & $0.43 \pm 0.15^{b}$ & $0.1 \pm 0.15^{b}$ & - \\
UM 461 & $0.69 \pm 0.10^{b}$ & $0.14 \pm 0.11^{b}$ & $2.09 \pm 0.15$ \\
He 2-10 & $0.50 \pm 0.06$ & $0.15 \pm 0.06$ & $1.92 \pm 0.16$ \\
IC 4662 & $0.59 \pm 0.06$ & $0.11 \pm 0.07$ & - \\
Mkn 178 & $0.57 \pm 0.12^{b}$ & $0.03 \pm 0.12^{b}$ & - \\
Mkn 1329 & $0.53 \pm 0.15$ & $0.25 \pm 0.16^{b}$ & $2.00 \pm 0.15$ \\
Haro 14 & $0.65 \pm 0.09$ & $0.03 \pm 0.10$ & - \\
\hline
\end{tabular}

${ }^{a}$ Corrected for galactic extinction (see Sect. 2.4.2); NIR colors are transformed to the 2MASS system (cf. Sect. 2.4.1). The $B$ band denotes the Johnson $B$. Errors include cumulative uncertainties in the determination of color profiles, the calibration and the transformation to the 2MASS system.

${ }^{b}$ Possible local instabilities in one SBP at low $S / N$ levels.

${ }^{c}$ Possible contamination by gas emission over a large portion of the LSB host galaxy.

The intense SF activity in Tol 3 is also reflected on relatively blue optical colors $(B-V=0.24, V-I=0.28$; Marlowe et al. $1997)$ in its nuclear region, as well as on copious $\mathrm{H} \alpha$ emission $\left(1.1 \times 10^{41} \mathrm{erg} \mathrm{s}^{-1}\right.$; Marlowe et al. 1997). Deep H $\alpha$ imaging by Marlowe et al. (1995) revealed a bipolar outflow roughly perpendicular to the major axis of the $\mathrm{BCD}$, extending out to $\sim 2.8 \mathrm{kpc}$ from its nuclear region.

Unsharp-masked NIR images reveal a complex morphology in the HSB regime of the BCD (inset in Fig. 2a). Regions A and B are immersed in an extended "S-shaped" pattern, $\sim 0.9 \mathrm{kpc}$ in length, ending at its NW tip with a curved feature ( $\mathrm{N}$ arc). There is some evidence for propagation of SF activities, as both archival optical NTT and NIR data reveal signatures of a younger age and stronger ionized gas emission towards region B (cf. Fig. 3, bottom). For region A we determine within a rectangular $4^{\prime \prime} \times 4^{\prime \prime}$ aperture colors of $J-H=$ $0.61\left(J-H^{\ddagger}=0.56\right)$ and $H-K=0.41\left(H-K^{\ddagger}=0.58\right)$. For knot B we infer a $J-H=0.39\left(J-H^{\ddagger}=0.14\right)$ and $H-K=$ $0.48\left(H-K^{\ddagger}=0.71\right)$. Such colors suggest a younger stellar age together with an appreciable ionized gas contribution towards the SE part of the SF component. The latter is verified from the $\mathrm{H} \alpha$ equivalent width ( $E W)$ map in Fig. 3 (bottom) (see also Gil de Paz et al. 2002) which shows that SE of knot B, and all over an extended rim perpendicular to the major axis of the $\mathrm{BCD}$, the $E W(\mathrm{H} \alpha)$ rises to $>200 \AA$.

Interestingly, unsharp-masked NIR and optical images reveal on larger scales a chain of faint knots (depicted with crosses in the inset of Fig. 2a), arranged over $\sim 2.5 \mathrm{kpc}$ SW of regions $\mathrm{A}$ and B. Their typical $J^{\ddagger}$ magnitudes of $\sim 20.6 \ldots 19$ mag translate into absolute magnitudes of $-10 \ldots-12 \mathrm{mag}$. The nature and formation history of this

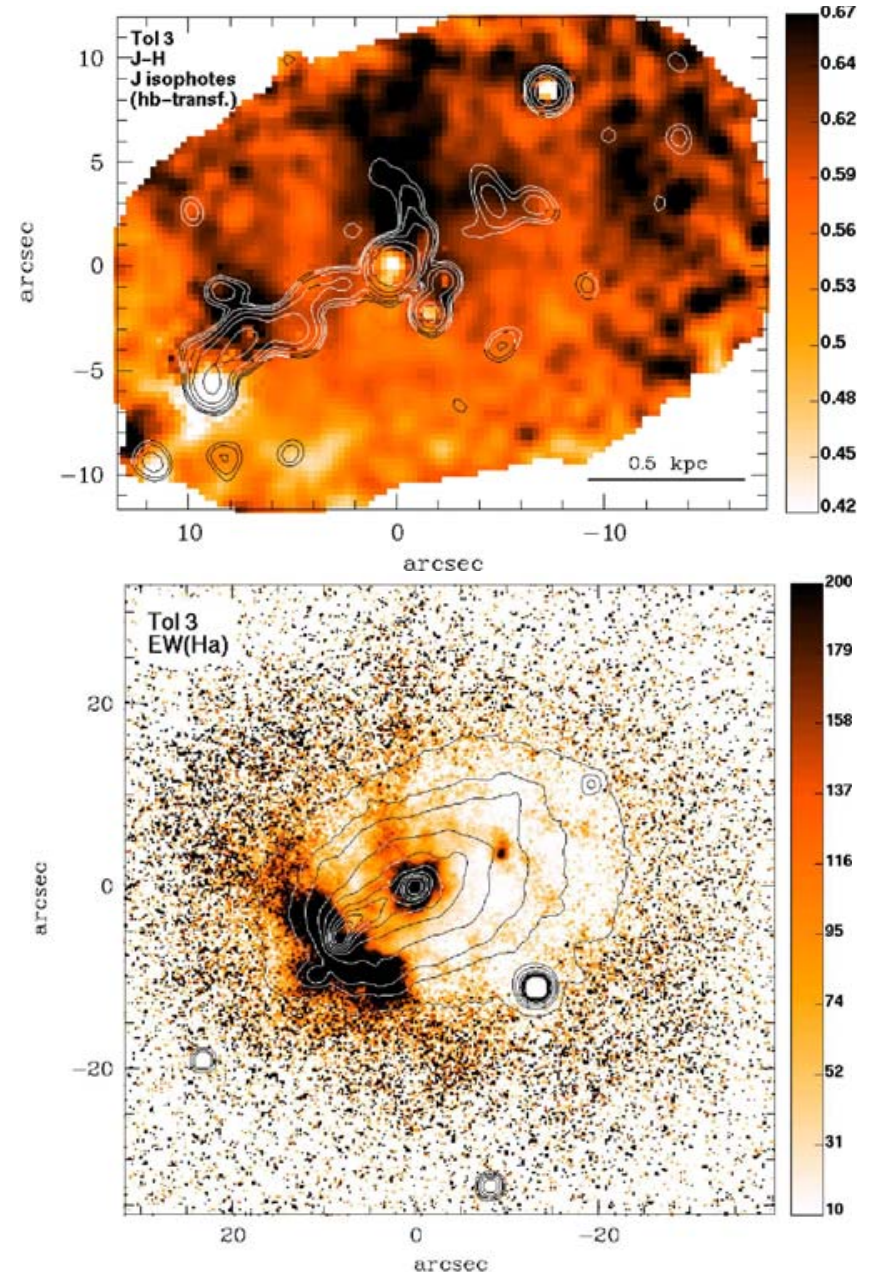

Fig. 3. Top: $J-H$ color map of the central region of Tol 3, corrected for Galactic extinction. The overlaid $J$ contours are computed from the contrast-enhanced blow-up included in Fig. 2 (see text for details). Bottom: $E W(\mathrm{H} \alpha)$ map of Tol 3, overlaid with $R$ contours.

extended feature is intriguing. One interpretation is that it delineates the approaching side of an oblate star-forming shell triggered by the burst, or that it may be associated with an inclined circumnuclear disk of $\sim 1 \mathrm{kpc}$ in radius. The first hypothesis is consistent with the findings by Alton et al. (1994), who suggested from imaging-polarimetry the presence of a largescale bipolar reflection dust-nebula illuminated by the central starburst region. This could provide effective UV-shielding, thereby allowing for secondary SF activity. The systematically redder colors in the NE half of Tol 3 (Fig. 3, top) are also in line with the same hypothesis, if they originate from the more strongly absorbed far side of the galaxy.

SBPs in the NIR (Fig. 2b) show in the radius range $22^{\prime \prime} \leq$ $R^{*} \leq 48^{\prime \prime}$ an exponential intensity fall-off with a scale length $\alpha=0.52 \mathrm{kpc}$. This value is close to the $B$ scale length of $\alpha=$ $0.48 \mathrm{kpc}$, inferred for the LSB component by Marlowe et al. (1997) within $\lesssim 30^{\prime \prime}$. By contrast, our surface photometry does not appear to be compatible to that of Kunth et al. (1988). These authors show optical SBPs out to a radius $R^{*}=80^{\prime \prime}$, by a factor of 1.6 larger than the study here or in Doublier et al. (1999) and up to 2.7 times larger than in Marlowe et al. (1997). NIR SBPs 

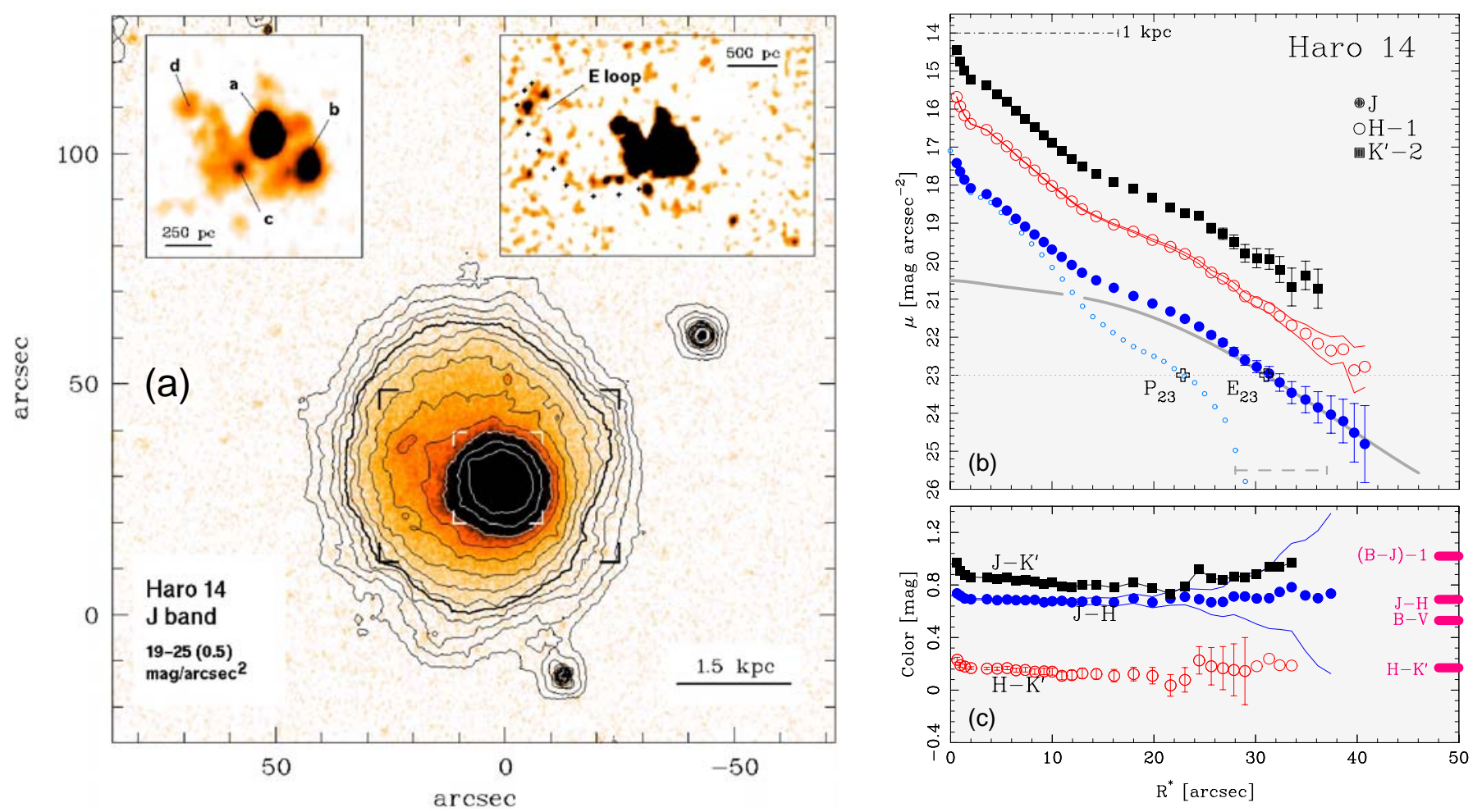

Fig. 4. Haro $14(D=12.5 \mathrm{Mpc})$. For explanations of symbols and labels, refer to Fig. 2. a) $J$ band image and isophotes. Note that the center of the star-forming regions is offset by $\sim 0.5 \mathrm{kpc}$ from the center of the outer circular isophotes. The upper-left and upper-right insets show unsharp-masked versions of the central part of the BCD, marked in the main image by the white and black brackets, respectively. In the upper-left inset we mark regions a through $\mathrm{d}$. The upper-right inset reveals an extended, curved chain of knots traceable up to $\sim 1.5 \mathrm{kpc}$ from the brightest region a. b), c) Surface brightness and color profiles. The thick grey line shows a fit to the LSB component using a modified exponential distribution Eq. (6) with $b, q=3.6,0.94$.

show no evidence for a dominant $R^{1 / 4}$ profile in Tol 3 (cf. Kunth et al. 1988, Doublier et al. 1999). A de Vaucouleurs profile is neither supported by the Sersic index $\eta=1.4$ we derive for the entire $J$ profile.

The NIR color profiles (Fig. 2c) show minor gradients $\left(<0.15 \mathrm{mag} \mathrm{kpc}^{-1}\right)$ and level off to $J-H=0.54 \mathrm{mag}$ and $H-K=0.12 \mathrm{mag}$ for $R^{*}>20^{\prime \prime}$. These results are not compatible to Doublier et al. (2001), who report for $R^{*}>10^{\prime \prime}$ a roughly linear $J-H$ color increase from $\sim 0.5 \mathrm{mag}$ to $\sim 2.0 \mathrm{mag}$. A good agreement is found with Vanzi et al. (2002), who derive for the LSB component of Tol 3 colors of $J-H=0.6$ mag and $H-K=$ $0.25 \mathrm{mag}$.

\subsection{Haro 14 (NGC 244)}

This relatively metal-rich $\mathrm{nE} \mathrm{BCD}\left(\mathrm{Z} \approx Z_{\odot} / 3\right.$, Hunter \& Hoffman 1999) shows SF activity ontop a smooth, nearly circular stellar LSB host galaxy (Fig. 4a). The intensity distribution of the latter (Fig. 4b) is approximated best by a modified exponential distribution (Eq. (6)), flattening for $R^{*} \lesssim 20^{\prime \prime}$ (see the detailed discussion in Sect. 5.2).

The central part of the BCD contains a massive complex of SF regions, displaced by $\sim 0.5 \mathrm{kpc} \mathrm{SW}$ of the geometrical center of the outer LSB isophotes. This SF component measures $\approx 1.4 \mathrm{kpc}$ in diameter in the $J$ band (this paper) and $\sim 2 \mathrm{kpc}$ in the $\mathrm{H} \alpha$ line (Marlowe et al. 1997). Its composition out of several individual regions, reported by Doublier et al. (1999) from optical images, is also evident from the contrast-enhanced NIR images (Fig. 4a, upper-left inset), which reveal a wealth of individual regions, spanning a range of $\gtrsim 3 \mathrm{mag}$. The upper-right inset of Fig. 4a reveals a chain of faint knots with a length of $\approx 1.5 \mathrm{kpc}$ (labeled "E loop"), extending eastwards from the main SF complex. There is a hint for a similar feature in the southwestern direction. Published data (e.g., the $\mathrm{H} \alpha$ image by Marlowe et al. 1997) do not allow to assess whether these faint features may trace induced star formation along supergiant shells, as might be hypothesized from their morphology.

For the two brightest regions, denoted a and $b$, we obtain respective absolute magnitudes of $M_{\mathrm{J}}{ }^{\ddagger}=-14.7 \mathrm{mag}$ and $-13.3 \mathrm{mag}$, and effective radii $<80 \mathrm{pc}$. If the mean $E(B-V)=0.35$ for Haro 14 (Hunter \& Hoffman 1999) applies to knots $\mathrm{a}$ and $\mathrm{b}$, then their de-reddened colors would be $J-H^{\ddagger} \sim 0.6$ and $H-K_{s}^{\ddagger} \sim 0.2$. Such colors are reached at the earliest when the NIR emission becomes dominated by red supergiants $(t \sim 10 \ldots 30 \mathrm{Myr})$, and suggest no strong nebular line contamination. This points against substantial ongoing star formation in regions $a$ and $b$.

The large spatial extent of SF sources in the inner portion of the BCD is further evidenced by the profile decomposition (Fig. $4 \mathrm{~b}$ ), yielding a plateau radius $P_{23}^{\mathcal{J}} \approx 1.4 \mathrm{kpc}$ in the $J$ band. The optical and NIR colors of the LSB host galaxy indicate an age of several Gyr, in agreement with previous estimates by Marlowe et al. (1999). 

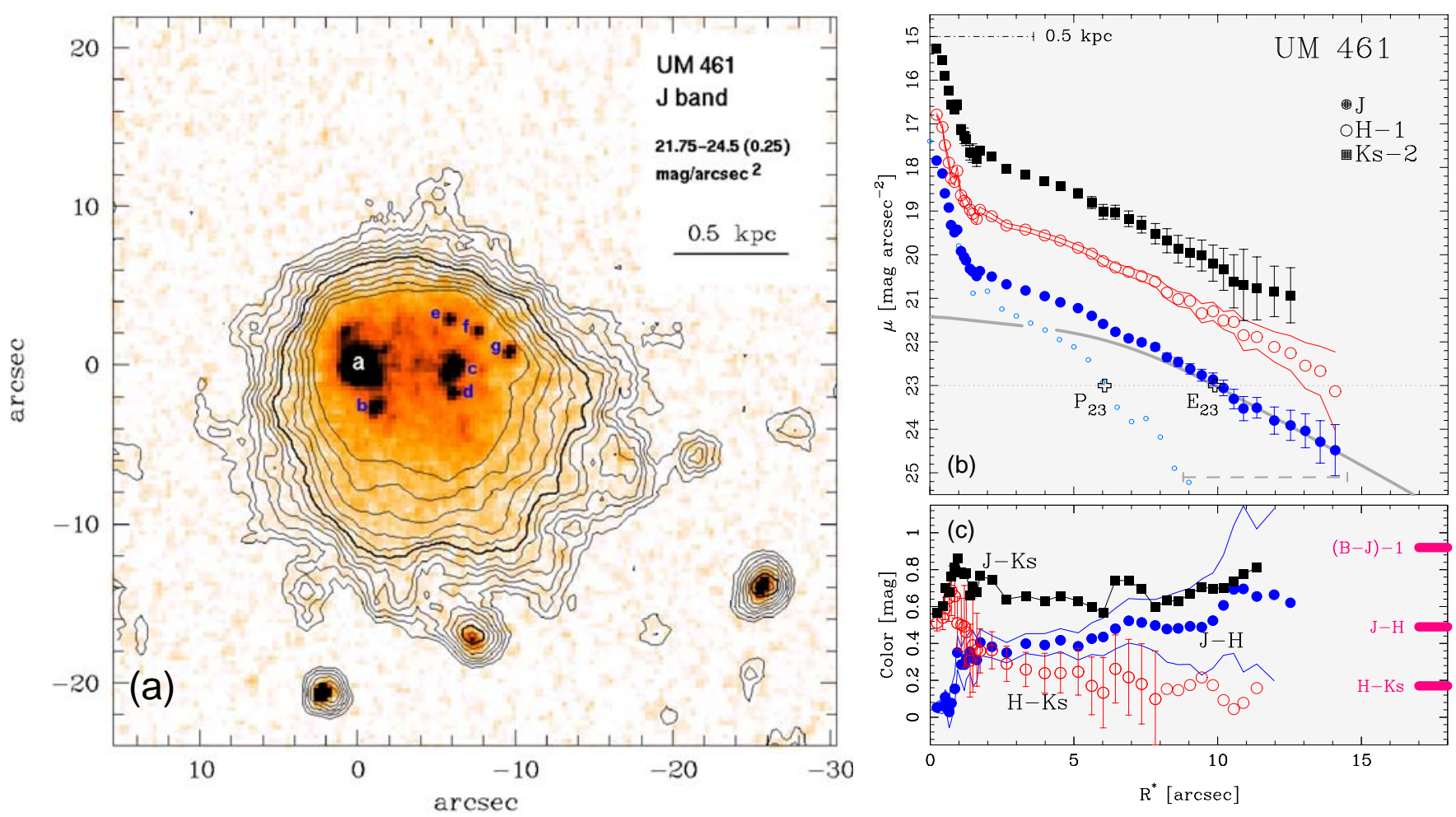

Fig. 5. UM $461(D=14.3 \mathrm{Mpc})$. For explanations of symbols and labels, refer to Fig. 2. a) $J$ band image and isophotes. Bright stellar assemblies in the central portion of the BCD are indicated. b), c) Surface brightness and color profiles. The thick grey line shows a fit to the LSB host galaxy using Eq. (6) with parameters $b, q=2.3,0.85$.

\subsection{UM 461}

Telles \& Terlevich (1995) suggested that the iI BCD UM 461 forms together with UM 463, UM 465 and UM 462 a loose group of dwarf galaxies.

SF activity is confined to the whole northeastern part of the galaxy, i.e. within the $21.5 \mathrm{~J} \mathrm{mag} / \square^{\prime \prime}$ isophote, or on a spatial scale of $\sim 0.9 \times 0.7 \mathrm{kpc}$. The two brightest SF regions a $\left(m_{J^{\ddagger}}=\right.$ $17.3 \mathrm{mag}$; Fig. 5a) and c ( $\left.m_{J^{\ddagger}}=19.5 \mathrm{mag}\right)$ in UM 461 are separated by $20 \mathrm{~km} \mathrm{~s}^{-1}$ in the velocity space. This difference is of the order of the intrinsic H I velocity dispersion within UM 461 ( $\sim 30 \mathrm{~km} \mathrm{~s}^{-1}$; van Zee et al. 1998). The available data allow us to resolve a manifold of morphological features within the SF component, most notably a chain of compact sources northwest of region c (labelled e-g) with a typical $M_{J}{ }^{\ddagger} \gtrsim-10.4$ mag. Their $J-H^{\ddagger}$ colors of $0.5-0.6$ mag are consistent with the interpretation by Méndez \& Esteban (2000) that they are extinguished SF regions, formed not earlier than $100 \mathrm{Myr}$ ago. From profile decomposition we infer the summed up luminosity fraction of compact and diffuse SF sources to $\sim 37 \%$ of the $J$ band light of UM 461.

UM 461 shows in its faint outskirts a slight asymmetry towards the SW direction. The intensity profile of the LSB host can be approximated by a med model with a scale length $\alpha=$ $0.21 \mathrm{kpc}$ and a depression parameter $q \approx 0.85$ (Fig. 5b). The $J$ band scale length derived here is in excellent agreement with the value derived in the optical by Telles et al. (1997). Color profiles reflect the ongoing SF for small radii $\left(R^{*} \lesssim 2^{\prime \prime}\right)$, and approach mean values of $J-H=0.49 \mathrm{mag}$ and $H-K_{\mathrm{s}}=$
0.17 mag in the LSB periphery. Such colors, together with the $B-J \approx 1.9$ mag determined from optical data, point consistently to a relatively evolved stellar LSB background. The NIR colors derived here do not appear to be compatible with those by Doublier et al. (2001). These authors find within the radius range $5^{\prime \prime} \lesssim R^{*} \lesssim 10^{\prime \prime}$ the $J-H$ color to increase from 1.5 to $\sim 2.3 \mathrm{mag}$, whereas the $H-K$ color shows a continuous decrease from $-0.5 \mathrm{mag}$ to $<-1 \mathrm{mag}$. Also, the integrated $J-H$ and $H-K$ colors of 0.99 mag and -0.68 mag, respectively, listed in Doublier et al. differ significantly from the values of 0.47 mag and 0.2 mag derived in the present study.

\subsection{Henize 2-10 (ESO 495-G21)}

This relatively metal-rich $\mathrm{iE} \mathrm{BCD}\left(Z_{\odot} / 2.4 \ldots \gtrsim Z_{\odot}\right.$; Schaerer et al. 1999; Kobulnicky et al. 1999) is the first extragalactic system in which the broad He II $\lambda 4686$ line was detected. The starburst nature of He 2-10 is evidenced by a chain of bright Super-Star Clusters (SSCs) in its brightest western SF region W (Conti \& Vacca 1994), extended X-ray and H $\alpha$ emission (Hensler et al. 1997, Papaderos \& Fricke 1998), as well as a large bipolar outflow from the SF region (Papaderos \& Fricke 1998), with an expansion velocity between $\sim 250$ and ¿360 $\mathrm{km} \mathrm{s}^{-1}$ (Méndez \& Esteban 1999; Johnson et al. 2000).

Unsharp masking (inset in Fig. 6a) reveals a wealth of morphological features in the nuclear region of He 2-10, most notably an extended feature protruding northwest of region $\mathrm{W}$ (labelled NW) and an arc-like chain of compact sources connecting the tip of region NW with the secondary SF knot E. 

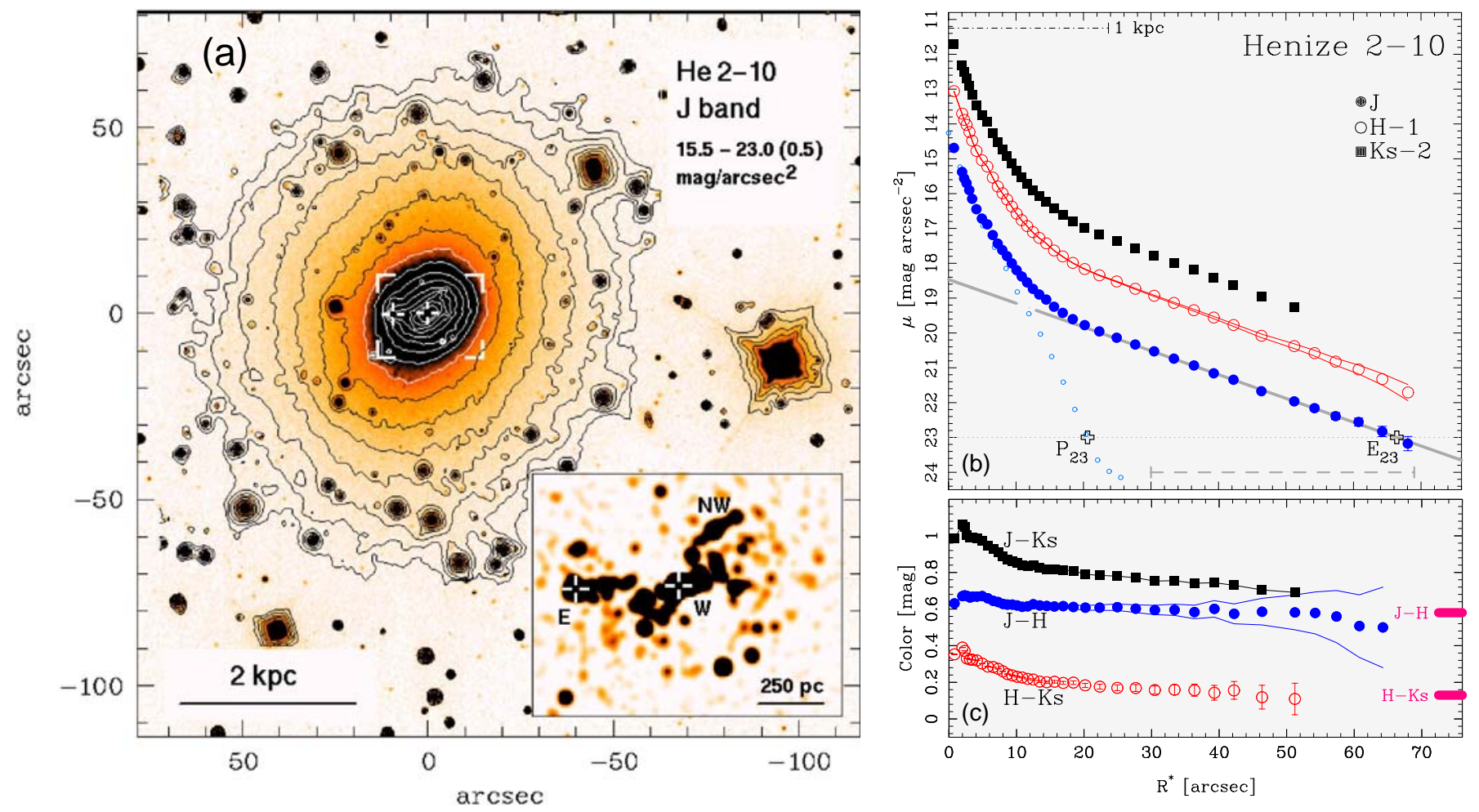

Fig. 6. Henize $2-10(D=8.7 \mathrm{Mpc})$. For explanations of symbols and labels, refer to Fig. 2. a) $J$ band image and isophotes. The inset shows an unsharp-masked version of the nuclear region of the BCD (region marked by the brackets in the main image). b), c) Surface brightness and color profiles.

Ground-based $B-R$ maps by Papaderos \& Fricke (1998) indicate that region NW and the concatenation of sources bending southwards of it are considerably bluer than the underlying LSB host galaxy.

Our SBPs (Fig. 6b) show an exponential intensity decrease in the LSB component $\left(R^{*} \gtrsim 20^{\prime \prime}\right)$, with a $J$ scale length identical to that obtained previously from optical data ( $\alpha=0.67 \mathrm{kpc}$, Papaderos \& Fricke 1998).

\subsection{Tol 1400-411 (NGC 5408)}

This cometary iI BCD is a member of the Cen A group (van den Bergh 2000), situated at a projected distance of $7.2^{\circ}$ $(\sim 0.6 \mathrm{Mpc})$ from Cen A. Intense SF activity is taking place mainly at the SW part of its elongated stellar LSB host, where ground-based NIR and archival $H S T$ /WFPC2 optical data (PI: P. Seitzer, G0-8601) reveal three bright $\left(m_{V} \lesssim 16.2\right)$ stellar clusters spread over $8^{\prime \prime}(\sim 200 \mathrm{pc})$. The appreciable ionized gas contribution in the SF component of Tol 1400-411 is reflected on the very blue $V-I$ color of $\$-0.4 \mathrm{mag}$, in the brightest cluster, A (cf. 1.h.s. blow-up of Fig. 7a) and the large $E W(\mathrm{H} \beta) \gtrsim$ $250 \AA$ A determined for this system by Masegosa et al. (1994).

Interferometric $21 \mathrm{~cm}$ line studies (Fritz 2000) show that the BCD is immersed within a large, rotationally supported H I cloud of about $8 \times 16 \mathrm{kpc}$ in size. The $\mathrm{HI}$ halo reveals two surface density maxima, one coinciding with region $A$ and the other one located $\sim 1^{\prime}$ to the east.

The present data, as well as $\mathrm{H} \alpha$ images by Fritz (2000), reveal signatures of low-level SF activity all over the $22 J \mathrm{mag} / \square^{\prime \prime}$ isophote of the BCD, i.e. $2.6 \mathrm{kpc}$ across. Interestingly, $30^{\prime \prime} \mathrm{NE}$ of region $\mathrm{A}$ our data show a nearly circular region, $\approx 12^{\prime \prime}$ in diameter, with relatively blue $(B-R$ of $\lesssim 0.75, V-I \lesssim 0.45 \mathrm{mag}$ ) colors in its unresolved interior (denoted B in Fig. 7a). Such colors, being significantly bluer than those in the LSB host $(B-R \approx 1, V-I=0.6-0.7)$, suggest that region $B$ is comparatively young. This is also suggested by the NIR colors $(J-K=0.7 \ldots 0.9)$ and luminosities $\left(M_{J}{ }^{\ddagger}=-8.6 \ldots-9.7\right)$ of bright sources therein which are consistent with a population of young (10-20 Myr) red supergiants (cf. e.g. Elias et al. 1985; Bertelli et al. 1994).

Another conspicuous feature seen at the northeastern part of the $\mathrm{BCD}$ is a comparatively blue $(B-R \approx 0.6)$ curved strip (region $C$ ), apparently bending from the NE tip of the LSB component to the north; a counter-feature of this region is probably present at the southern part of the BCD.

The large extent of the SF component, as well as several bright foreground stars in the periphery of Tol 1400-411, render the determination of the structural properties and color of its LSB component difficult. The SBPs (Fig. 7b) are approximated best with Eq. (6) with $(b, q)=(3.0,0.8)$. The mean LSB colors, $J-H=0.42, H-K_{\mathrm{s}}=0.28$ and $V-I=0.6-0.7$ can be brought into rough agreement, given the uncertainties discussed in Sect. 3.5. The GALEV model (see Sect. 5.4) yields for a metal-poor stellar population forming in a single burst or continuously an age between several $10^{8}$ to a few $10^{9} \mathrm{yr}$ (cf. Fig. 17). However, these colors might be influenced by ionized gas emission and the younger stellar populations in the NE boundary. 

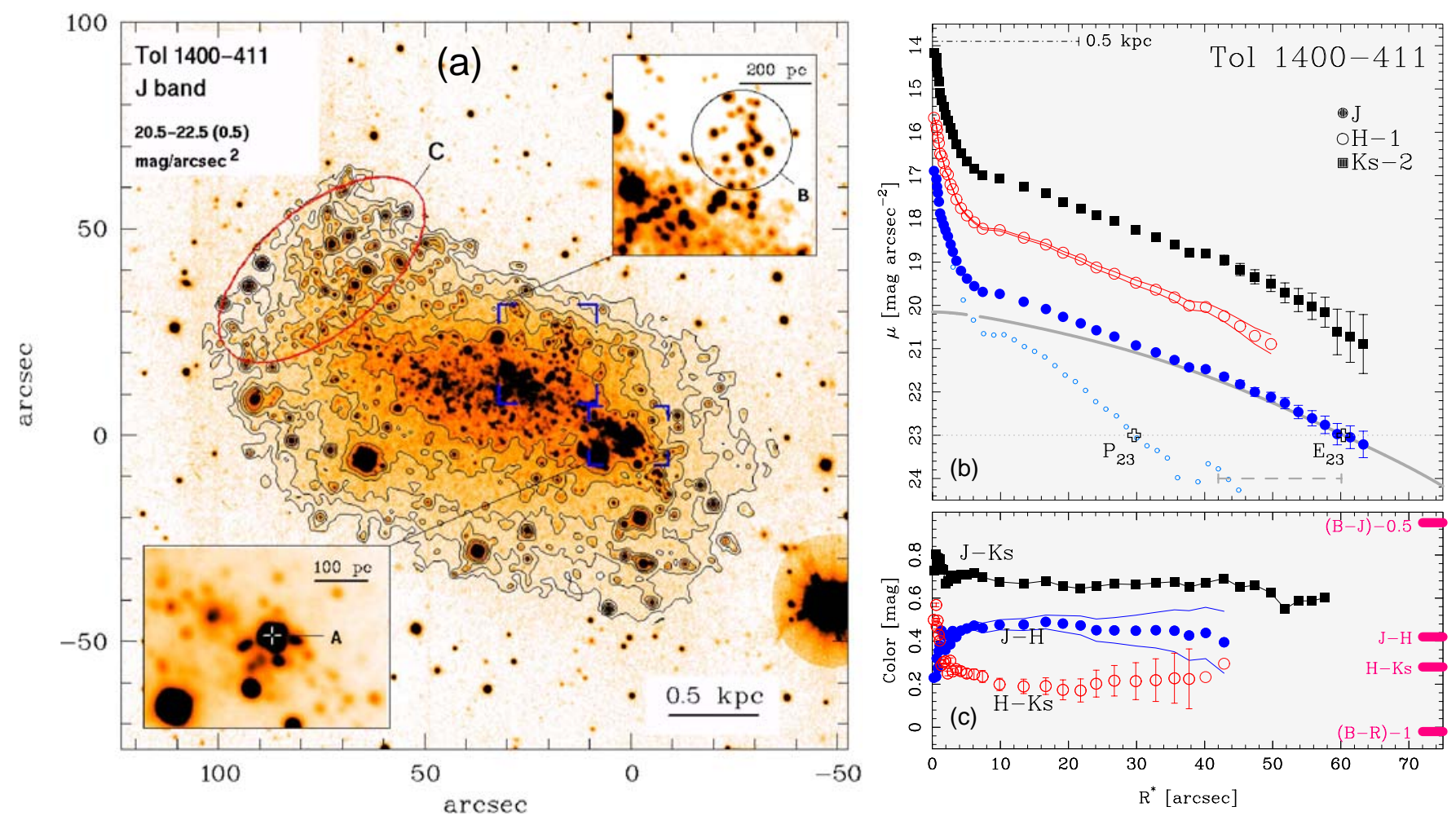

Fig. 7. Tol 1400-411 $(D=4.8 \mathrm{Mpc})$. For explanations of symbols and labels, refer to Fig. 2. a) $J$ band image and isophotes. The insets show magnifications of the bright SW star-forming knot A as well as the region B extending northwestwards from the major axis. The bluer NE region $\mathrm{C}$ is marked. b), c) Surface brightness and color profiles. The thick grey line shows a fit to the host galaxy using Eq. (6) with $b, q=$ $3.0,0.82$.

\subsection{Pox 4}

Despite its moderate metal deficiency $\left(Z_{\odot} / 9.3 \ldots Z_{\odot} / 7.6 ;\right.$ Kunth $\&$ Sargent 1983; Vacca \& Conti 1992), Pox 4 is a relatively luminous $\left(M_{B}=-18.81\right)$ iI BCG (Méndez \& Esteban 1999). It is accompanied by a faint SF dwarf galaxy, Pox $4 \mathrm{~B}$, at a projected distance of $5 \mathrm{kpc}$ and a velocity difference of $130 \mathrm{~km} \mathrm{~s}^{-1}$. On our NIR images, we do not detect any emission in between Pox 4 and Pox $4 \mathrm{~B}$ down to an approximative surface brightness level of $24 J \mathrm{mag} / \square^{\prime \prime}$ (Fig. 8a).

The optical morphology of Pox 4 is dominated by SF regions and extended ionized gas emission on a spatial scale of $5 \times 2 \mathrm{kpc}$ (cf. Figs. 1 and 2 in Méndez \& Esteban 1999). A substantial color shift due to intense $(E W(\mathrm{H} \alpha)=1410 \AA)$ nebular line emission has been observed in the brightest assembly of SF sources M9 (inset of Fig. 8a) for which Méndez $\&$ Esteban (1999) report a blue ( $-0.8 \mathrm{mag}) U-B$ together with an extremely red $(+0.7 \mathrm{mag}) B-V$ color. The NIR colors of region M9 $\left(J-H^{\ddagger}=0.28, H-K_{\mathrm{s}}{ }^{*}=0.47\right)$ are also suggestive of strong ionized gas contamination. The latter is also reflected on the color profiles (Fig. 8c), approaching for $R^{*} \lesssim 3^{\prime \prime}(0.7 \mathrm{kpc})$ colors as extreme as $J-H=0.2$ mag together with $H-K_{\mathrm{s}}=$ 0.6 mag.

The SF morphology in Pox 4 is intriguing. Unsharp masking reveals eastwards of region M9 a ring-like distribution of compact sources with a projected size of $3.2 \times 1.5 \mathrm{kpc}$ and a position angle of $\sim 124^{\circ}$. A similar morphology has been reported from optical data by Méndez \& Esteban (1999), who interpreted Pox 4 as a Cartwheel-like galaxy, downscaled by 1-2 orders of magnitude.

The available deep NIR data allow us to detect at faint intensities $\left(\approx 22.5 \mathrm{~J} \mathrm{mag} / \square^{\prime \prime}\right)$ a smooth underlying LSB host galaxy. Its $J_{\text {LSB }}$ profile can be approximated with Eq. (6) and a depression parameter $q=0.9$ (Fig. 8b). From profile decomposition we infer the absolute $J$ magnitude of this stellar host to $-18.2 \mathrm{mag}$, which translates to $M_{B}>-17.2 \mathrm{mag}$ for a $B-J>$ 1. Thus, despite its high total luminosity, Pox 4 qualifies by the absolute magnitude of its LSB host as a dwarf galaxy.

Our SBPs show some similarity to the uncalibrated profiles in Telles et al. (1997) out to $R^{*}=15^{\prime \prime}$. However, the scale length derived here for $10^{\prime \prime} \leq R^{*} \leq 16^{\prime \prime}$ for the LSB component $\left(\alpha=3{ }^{\prime \prime} 8=0.86 \mathrm{kpc}\right)$ is twice as large as that in Telles et al. (1997).

\subsection{Pox $4 B$}

This intrinsically faint $\left(M_{J}=-16.2 \mathrm{mag}\right)$ galaxy has been suggested by Méndez \& Esteban (1999) to have triggered the starburst activity in Pox 4 through a face-on collision. As evidenced by faint $\mathrm{H} \alpha$ emission (Méndez \& Esteban 1999), Pox $4 \mathrm{~B}$ still maintains a mild SF activity. The latter is probably taking place in three compact $\left(\sim 00^{\prime \prime} 5\right)$ sources, discernible in the central part of the galaxy. Pox 4 B shows little morphological distortions, except for a slight eastward extension of its LSB component (Fig. 8a). 


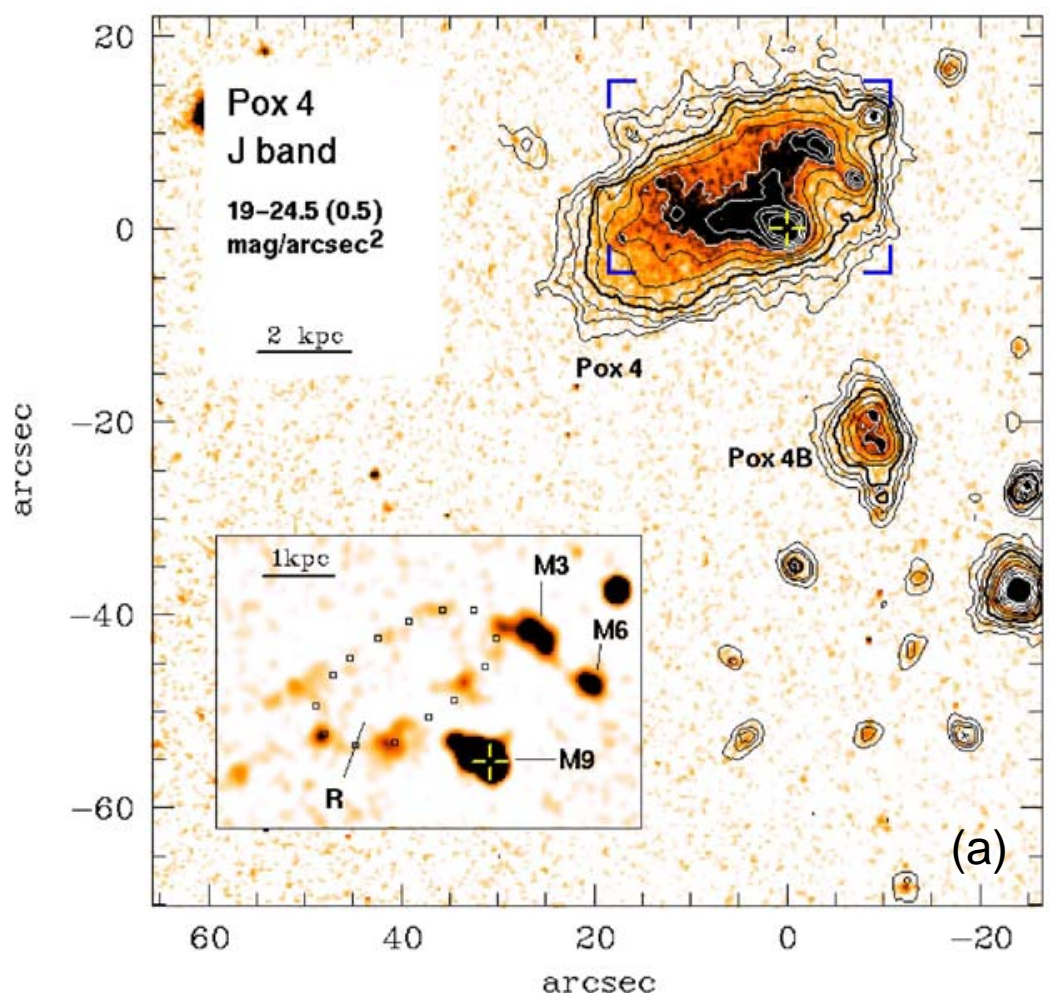

Fig. 8. Pox 4 and its companion Pox $4 \mathrm{~B}(D=$ $46.7 \mathrm{Mpc}$ ). For explanations of symbols and labels, refer to Fig. 2. a) $J$ band image and isophotes. The inset shows an unsharp-masked close-up of the SF regions in Pox 4 (area within brackets in the main image). M3, M6 and M9 mark the brightest $\mathrm{H}$ II regions referred to as 3, 6 and 9 by Méndez \& Esteban (1999). The ellipse of small boxes marks the ring of SF regions described by the same authors. In our data, M9 resolves into 3 knots arranged over a projected length of $3{ }^{\prime \prime} .2$ $(750 \mathrm{pc})$. b)-e) Surface brightness and color profiles of Pox 4 (b), c)) and Pox 4B (d), e)). The thick grey lines show decomposition fits to the stellar LSB host galaxies by means of modified exponential distributions (see Sect. 3.2), with $b, q=$ 2.7, 0.90 for Pox 4 and $b, q=2.0,0.80$ for Pox 4B.
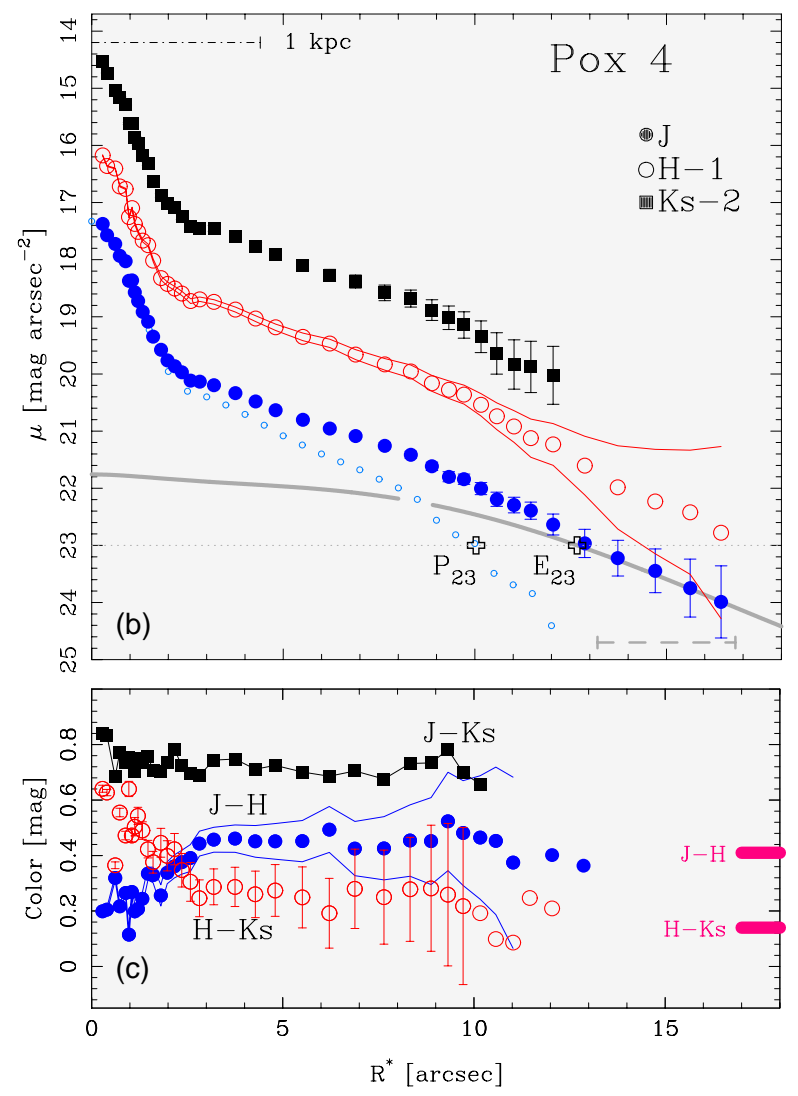

The SBPs (Fig. 8d) of Pox $4 \mathrm{~B}$ show for $2^{\prime \prime} \leq R^{*} \leq 5^{\prime \prime}$ an exponential fall-off with an $\alpha \approx 0.29 \mathrm{kpc}$, and a flattening for $R^{*}<2^{\prime \prime}$. We have verified that the latter intensity regime is not due to seeing. For this purpose, we convolved artificial exponential 2D models with the observed $\alpha$ and extrapolated central
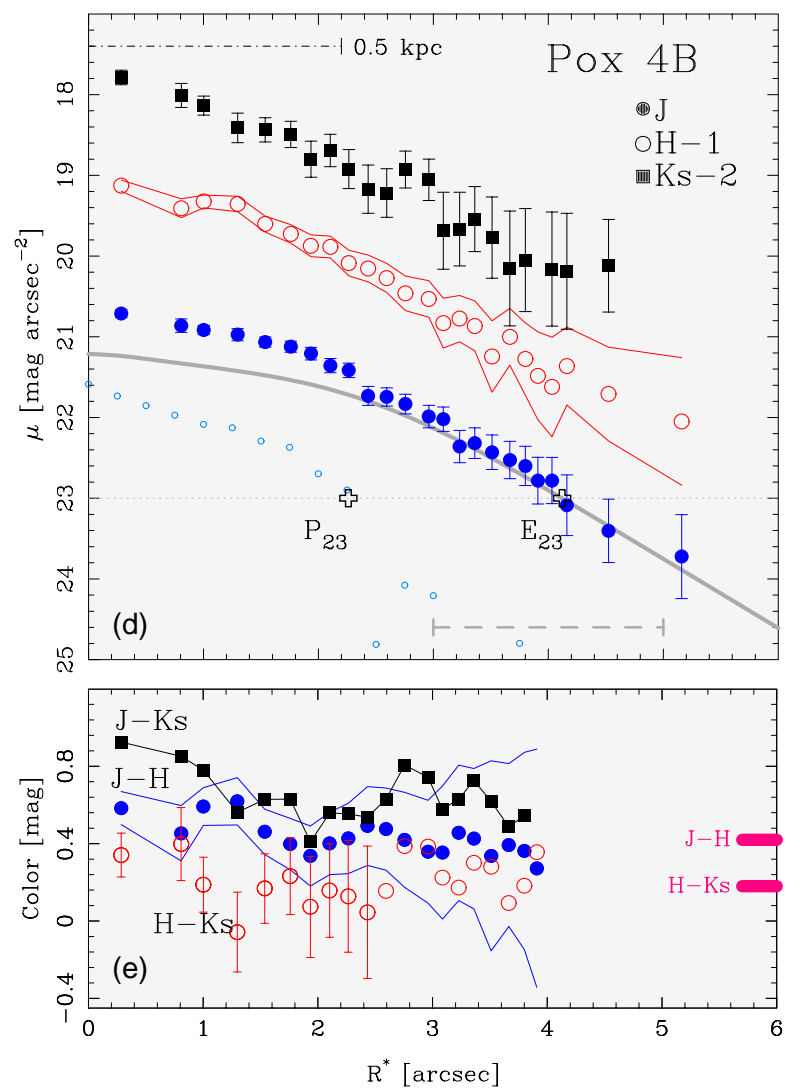

surface brightness $\left(\mu_{\mathrm{E}, 0} \approx 19.5 \mathrm{~J} \mathrm{mag} / \square^{\prime \prime}\right)$ with a Gaussian kernel matching the PSF of the coadded images $(\approx 0$.'7 FWHM). The resulting SBPs deviate only slightly from a pure exponential, even when the $F W H M$ is further degraded by a factor of 2 , and can by no means reproduce the strong flattening we detect 

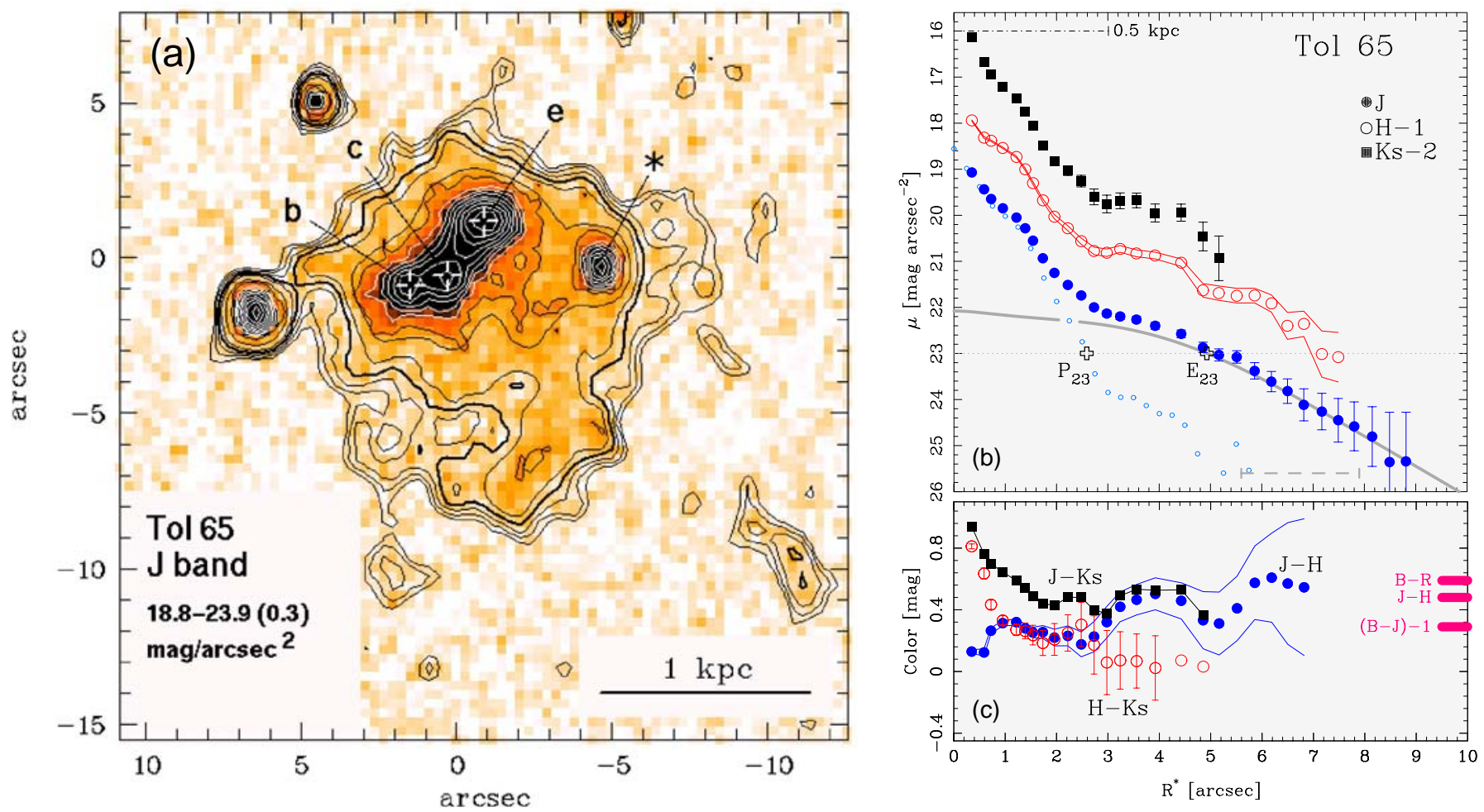

Fig. 9. Tol $65(D=34.2 \mathrm{Mpc})$. For explanations of symbols and labels, refer to Fig. 2. a) $J$ band image and isophotes. The sources b, c and e along the NE-chain of SF regions are marked, following the notation of P99. The feature marked by an asterisk is a red $\left(J-K_{\mathrm{s}} \approx 0.8\right.$ mag) compact source probably not belonging to the galaxy. The faint $\left(m_{J} \approx 20.8 \mathrm{mag}\right)$ galaxy "G1" (cf. P99) is visible to the SW. b), c) Surface brightness and color profiles. The thick grey line shows a decomposition fit to the host galaxy by means of a modified exponential distribution (see Sect. 3.2) with $b, q=2.7,0.90$.

in the SBPs of Pox 4B. In addition, a perfect exponential distribution with the $\mu_{\mathrm{E}, 0}$ and $\alpha$ quoted above would yield a $16 \%$ larger luminosity than the one actually measured for Pox 4B.

The available data do not allow us to study the NIR colors in spatial detail. The mean $J-H$ and $H-K_{\mathrm{s}}$ of respectively $\approx 0.4$ and $\approx 0.2$ are comparable to those of the LSB component of Pox 4 .

\subsection{Tol 65}

Star formation in this very metal-deficient BCD $\left(Z_{\odot} / 24\right.$; Kunth \& Sargent 1983; Masegosa et al. 1994; Izotov et al. 2001) is taking place mainly in a chain of five compact $\left(1{ }^{\prime \prime} 2 \lesssim \varnothing \lesssim 2^{\prime \prime}\right)$ sources located at the NE part of an irregular, blue LSB envelope (P99). The combination of an extraordinarily blue $J-H^{\ddagger}$ $(\sim 0.1 \mathrm{mag})$ with a red $H-K^{\ddagger}(\approx 0.8 \mathrm{mag})$ color in the surroundings of the brightest SF region e (Fig. 9a) points to a substantial contribution of ionized gas emission. This is also suggested by the combination of a blue $U-B(\sim-1.05 \mathrm{mag})$ with a moderately red $B-R(\sim 0.4-0.5 \mathrm{mag})$ color derived by $\mathrm{P} 99$ all over the NE half of Tol 65, several 100 pc away from the opposite tips of the SF chain. Further evidence for intense ionized gas emission is provided by Keck spectroscopy by Izotov et al. (2001), who derived a large $E W(\mathrm{H} \alpha)$ of $>1000 \AA$ in the SF component of the BCD.

Ionized gas contamination appears to be small midway in the SF chain. The $B-R=0.15 \mathrm{mag}$ (P99), together with
$J-H^{\ddagger}$ and $H-K_{\mathrm{s}} *$ of 0.44 and $0.2 \mathrm{mag}$, respectively, observed in region $\mathrm{C}$ are consistent with a single burst stellar age of $\$ 100$ Myr. Note that the $J-H$ color in region $\mathrm{C}$ is barely bluer than the average value for the LSB component ( $0.48 \mathrm{mag}$ ). This is also true for the $H-K_{\mathrm{s}}$ color, which could be constrained at $R^{*} \gtrsim P_{23}^{\mathcal{J}}$ to $\lesssim 0.2 \mathrm{mag}$ (cf. Fig. 9c).

Deep optical surface photometry ( $\left.\mu \gtrsim 28.5 B \mathrm{mag} / \square^{\prime \prime}\right)$ for Tol 65 has first been presented in P99. These authors found optical SBPs to show an outer exponential regime for $R^{*}>7^{\prime \prime}$ and a flattening relative to the exponential fit inwards of $R^{*} \approx$ $3^{\prime \prime}$. In order to adequately decompose the optical SBPs, they modelled the LSB component with Eq. (6) and a depression parameter $q \gtrsim 0.8$. The type $\mathrm{V}$ profile of the LSB component is better visible in NIR wavelengths, where an even stronger flattening is required $(q \approx 0.9)$ to fit the data. The $J$ SBP shows in the radius range $5^{\prime \prime} .0 \leq R^{\star} \leq 77^{\prime \prime} 3$ an exponential intensity fall-off with a scale length $\alpha=0.28 \mathrm{kpc}$, in good agreement with the value derived in P99 ( $\alpha=0.26 \mathrm{kpc})$. The average LSB colors of $J-H=0.48 \pm 0.17$, together with a $B-R \lesssim 0.6 \mathrm{mag}$ and $B-J \lesssim 1.3 \mathrm{mag}$, inferable from the SBPs in P99, are consistent with a stellar age $\lesssim 1 \mathrm{Gyr}$, assuming an instantaneous SF process (cf. Sect. 3.5).

\section{9. $\mathrm{Tol} 1214-277$}

This metal-poor $\left(Z_{\odot} / 25\right.$; F01, Izotov et al. 2001) cometary iI BCD undergoes strong SF activity at the NE end of an 


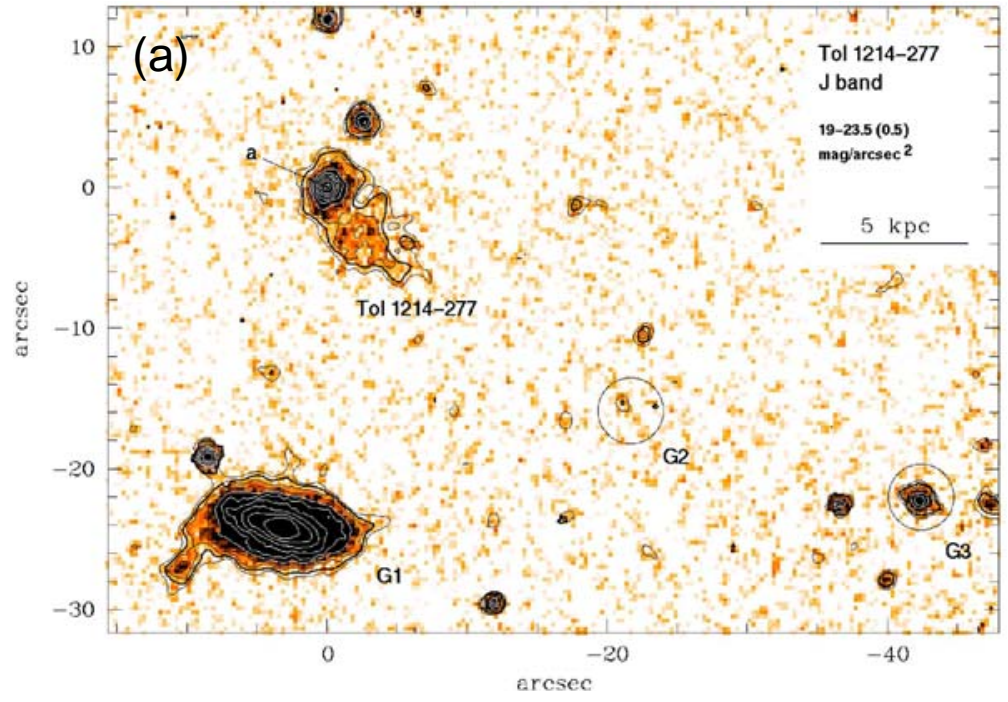

Fig. 10. Tol 1214-277 ( $D=102.6 \mathrm{Mpc})$. For explanations of symbols and labels, refer to Fig. 2. a) $J$ band image and isophotes. The bright star-forming complex at the NE edge of the galaxy (a) is marked, along with the faint galaxies G1-G3 (see F01). b), c) Surface brightness and color profiles. The thick grey line shows a decomposition fit to the host galaxy by means of Eq. (6) with $b, q=3.3,0.92$.
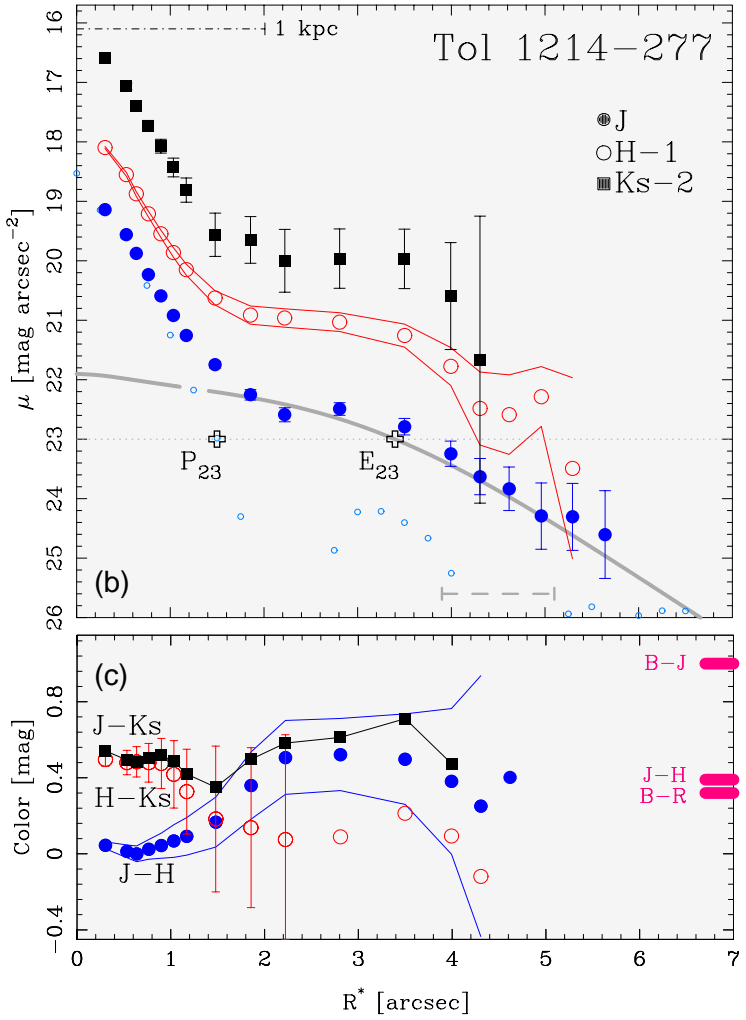

elongated stellar LSB body with an apparent size of $\approx 6.9 \times$ $3.4 \mathrm{kpc}$. The brightest SF complex (labelled a in Fig. 10a) contributes nearly one half of the BCD's optical light within the $25 \mathrm{Bmag} / \square^{\prime \prime}$ isophote (F01). The $J^{\ddagger}$ magnitude determined for region a, $18.6 \mathrm{mag}$, shows that in the NIR the starburst still provides $\sim 1 / 3$ of the galaxy's total flux. The colors of this region $\left(J-H^{\ddagger}=-0.02 \mathrm{mag}\right.$ and $\left.H-K_{\mathrm{s}}{ }^{\ddagger}=0.51 \mathrm{mag}\right)$ are consistent with a young burst age of $<7 \mathrm{Myr}$ for the BCD (see also F01).

Only the $J$ band SBP of Tol 1214-277 could be observed with a sufficient quality for a decomposition (Fig. 10b). Similar to Tol 65, it shows a type $\mathrm{V}$ distribution, as already reported from deep optical VLT FORS I data by F01. Fitting Eq. (6) to the $J_{\mathrm{LSB}} \mathrm{SBP}$, with the $(b, q)$ parameters fixed to the values derived in F01 $(3.3,0.92)$, we obtain a scale length $\alpha=0.53 \mathrm{kpc}$, slightly larger than that in the optical ( $\alpha=0.49 \mathrm{kpc} ; \mathrm{F} 01)$.

Fricke et al. (2001) reported for Tol 1214-277 nearly constant $U-B$ and $B-R$ colors of respectively -0.42 mag and 0.34 mag over an intensity span of 8 mag (colors adapted to the galactic absorption assumed here). Such colors, together with the $J-H \approx 0.4$ mag and $B-J \approx 1.1 \mathrm{mag}$ derived here, are slightly bluer than those of Tol 65, supporting the hypothesis that Tol 1214-277 is a relatively unevolved dwarf galaxy.

\subsection{Mkn 178 (UGC 6541)}

The spectroscopic properties of this intrinsically faint $\left(M_{B}=\right.$ $-13.9 \mathrm{mag}$ ) iE BCD, a member of the CVn cloud I of galaxies (Makarova et al. 1998), have been investigated in e.g. González-Riestra et al. (1988) and Guseva et al. (2000). Optical images reveal a complex morphology in the SF component, notably a pronounced separation of HSB regions into a compact assembly of bright knots to the SE (regions a and b) and an extended arc-like segregation of fainter sources to the NE. NIR images, on the contrary, show a more regular morphology, with only a few prominent sources in the central part of Mkn 178 (see Fig. 11a). Region a $\left(m_{J}^{*}=17.5\right.$ mag; cf. inset in Fig. 11a) coincides with the optically brightest region in the BCD and is the main locus of active star formation. The brightest NIR source, $\mathrm{b}\left(m_{J}^{\ddagger}=16.5 \mathrm{mag}\right)$, is optically faint and shows nearly the same $B-R$ color as the surrounding diffuse emission within the plateau component (cf. Papaderos et al. 2002). Sources $C$ and $d$ are immersed within the northern featureless region. The non-detection of an optical counterpart for region $d$, and the overall optical/NIR morphology of the SF component are suggestive of inhomogeneous, large-scale dust absorption on a spatial scale of $\sim 1 \mathrm{kpc}$. This may cause the apparent separation of the SF component in two large detached complexes and hide knot $d$ in optical wavelengths.

González-Riestra et al. (1988) and Guseva et al. (2000) have shown ionized gas emission to be negligible in region $b$ $(E W(\mathrm{H} \beta)=24 \ldots 34 \AA)$. By the color excess of $\mathrm{E}(B-V)=0.25$ given in González-Riestra et al. (1988), its observed $B-R$ color (0.67 mag, Papaderos et al. 2002) transforms to $0.28 \mathrm{mag}$, suggesting a single-burst age of $\lesssim 100 \mathrm{Myr}$. This is consistent with the colors of $J-H^{\ddagger}=0.24, H-K^{\prime}=0.06$ and $B-J^{\ddagger}=$ 1.6 mag obtained in the present work, if they are de-reddened adopting the same amount of intrinsic extinction. The presence of stellar complexes with an age of the order of $\sim 10^{8} \mathrm{yr}$ is in line with the detection of numerous luminous AGB stars in NIR color-magnitude diagrams (Schulte-Ladbeck et al. 2000), from which these authors infer significant SF activity over the last few $10^{8}$ yr. 

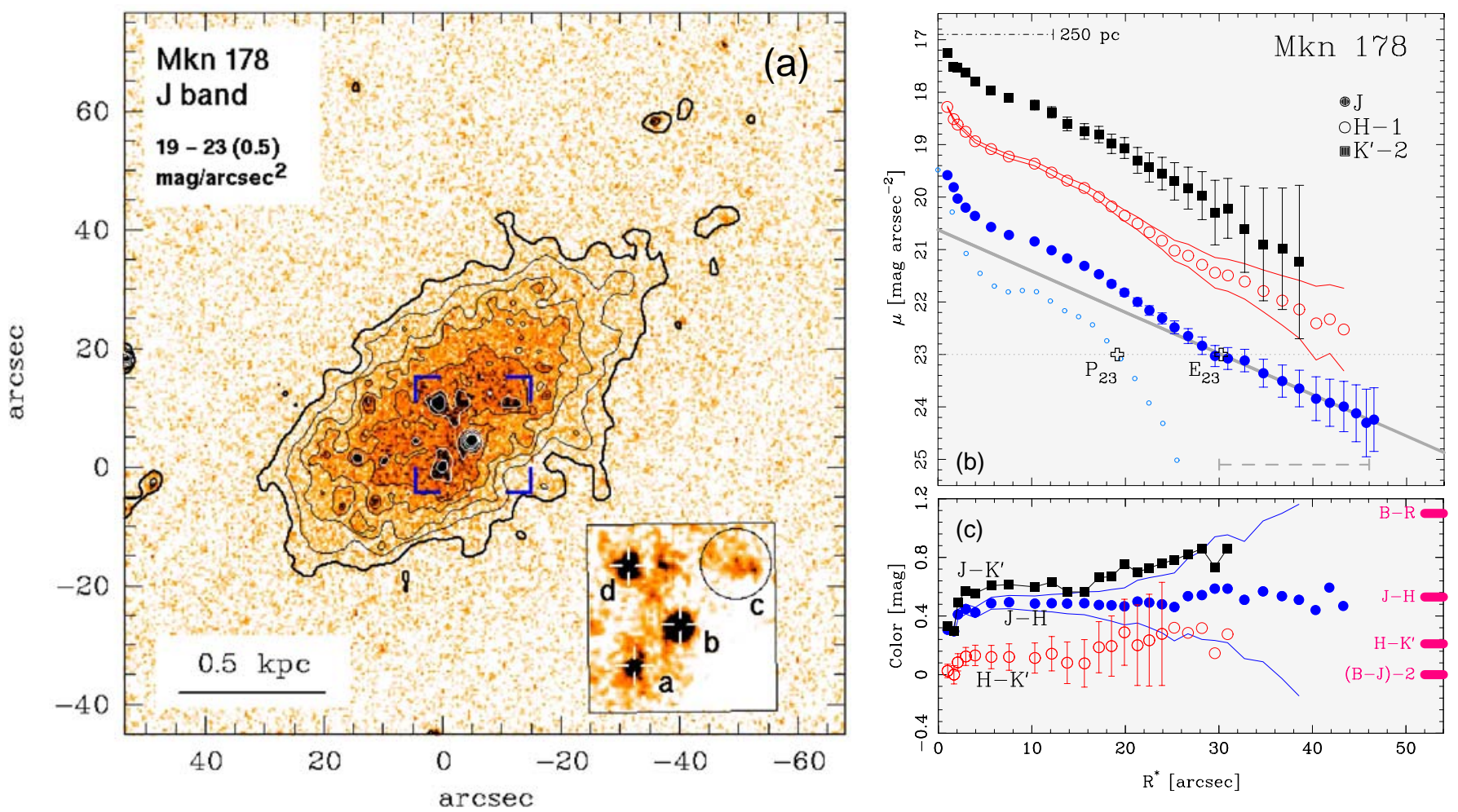

Fig. 11. Mkn $178(D=4.2 \mathrm{Mpc})$. For explanations of symbols and labels, refer to Fig. 2. a) $J$ band image and isophotes. The inset shows a close-up of the star-forming regions (delimited by brackets in the main image). The regions a-d described by Gonzalez-Riestra et al. (1988) and Papaderos et al. (2002) are labeled. b), c) Surface brightness and color profiles.

NIR SBPs of Mkn 178 (Fig. 11b) resemble closely the optical ones (Papaderos et al. 2002), showing an extended plateau component ontop a smooth, exponential LSB host galaxy. For the latter we derive a $J$ band scale length of $0.28 \mathrm{kpc}$ and colors $\left(J-H \approx 0.53, H-K^{\prime} \approx 0.2, B-J \approx 2.0\right.$ and $\left.B-R \approx 1.1 \mathrm{mag}\right)$ that consistently indicate an old stellar LSB background.

\subsection{Mkn 1329 (IC 3589/91, UGC 7790, VCC 1699)}

This relatively metal-rich $\left(Z_{\odot} / 5\right.$, Guseva et al. 2000) cometary iI BCD $\left(M_{B} \approx-16.8\right.$, Yasuda et al. 1997) is associated with a group of 11 galaxies (LGG 296, García 1993) within the Virgo Cluster.

As shown in the contrast-enhanced image (inset in Fig. 12a), numerous irregular concentrations are present in the inner zone of its elongated stellar LSB component, over a projected length of $\sim 3.5 \mathrm{kpc}$. An $\mathrm{H} \alpha$ exposure by Gallagher \& Hunter (1989) shows that SF activity is almost exclusively confined to a bright $\mathrm{H}$ II region (marked with a cross in Fig. 12a) at the SW end of the high-surface brightness component. The $\mathrm{H} \alpha$ flux derived for that SF region by the latter authors yields, at the distance adopted here, an $\mathrm{H} \alpha$ luminosity of $\approx 1.5 \times 10^{40} \mathrm{erg} \mathrm{s}^{-1}$ and a star formation rate (SFR) of $\sim 0.1 M_{\odot} \mathrm{yr}^{-1}$. The dominant SF region in Mkn 1329 is identifiable with a compact $(\varnothing \lesssim$ $300 \mathrm{pc})$, luminous $\left(M_{J}=-14\right)$ NIR source; its colors, $(J-H)^{\ddagger}=$ 0.16 and $\left(H-K_{\mathrm{S}}\right)^{\ddagger}=0.51$, are probably significantly influenced by nebular emission $(E W(\mathrm{H} \beta)=276 \AA$, Guseva et al. 2000).

The morphology of the SF regions and the high luminosity of the SW complex in Mkn 1329 are typical among cometary iI BCDs. The similarity of these systems with respect to the morphology of their LSB and SF component suggests a comparable evolutionary state/history or common mode of star formation. Propagation of SF activities along those objects' major axes has been proposed and observationally supported by a number of recent studies (cf. Noeske et al. 2000 and references therein).

The SBPs of Mkn 1329 (Fig. 12b) indicate a moderate central flattening of the LSB component, which can be approximated both by a Sérsic law with $\eta_{\mathrm{LSB}} \approx 0.7$, or a med with $b, q=(1.6,0.7)$ (see Table 2 and Sect. 3.2). The detection of a moderate central flattening in the LSB component owes to the comparatively small contribution of the superposed younger stellar population in Mkn $1329(\approx 17 \%$ of the total $J$ band light). The NIR colors in the outskirts of Mkn 1329, $(J-H)^{\ddagger}=$ $0.64,\left(H-K_{\mathrm{S}}\right)^{\ddagger}=0.06$, suggest an evolved stellar LSB component. Adopting a $B-J$ color characteristic of a few Gyr old stellar population, we can estimate the $B$ band structural parameters from the $\mu_{\mathrm{E}, 0}, \alpha$ and $M_{\mathrm{LSB}}$ determined from $J$ SBPs. We find that the host galaxy of Mkn 1329 shows, similar to other cometary BCDs (Noeske et al. 2000), structural properties intermediate between extended $\mathrm{dIs} / \mathrm{dEs}$ and compact $\mathrm{iE} / \mathrm{nE}$ BCDs.

\subsection{IC4662 (ESO 102-G014, He 2-269)}

This nearby $(D=2 \mathrm{Mpc}$; Heydari-Malayeri et al. 1990) dwarf galaxy has been studied spectroscopically by e.g. Pastoriza \& Dottori (1981), Stasinska et al. (1986), 

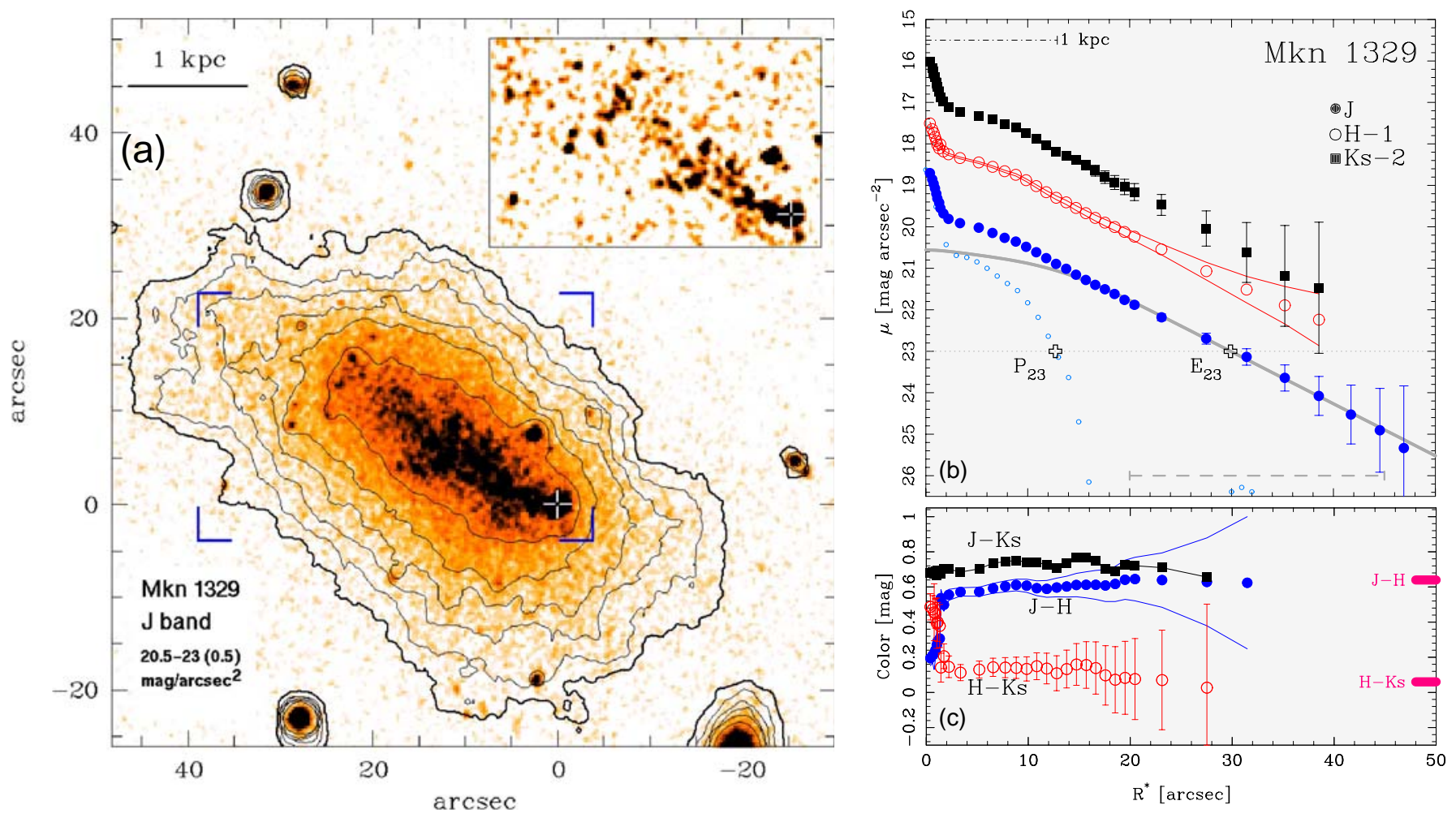

Fig. 12. Mkn $1329(D=16 \mathrm{Mpc})$. For explanations of symbols and labels, refer to Fig. 2 . a) $J$ band image and isophotes. inset: $h b$-transformed image of the central area (delimited by the brackets in the main image), showing the distribution of the bright regions along the major axis. The white cross marks the bright SW star-forming region for orientation. b), c) Surface brightness and color profiles. The thick grey line shows a decomposition fit to the host galaxy by means of a modified exponential distribution, Eq. (6), with $b, q=1.6,0.70$.

Heydari-Malayeri et al. (1990) and Hidalgo-Gámez et al. (2001). The latter authors derived the oxygen abundance in the brighter SF regions $\mathrm{A} \& \mathrm{~B}$ to $Z_{\odot} / 6.5 \ldots Z_{\odot} / 7.6$, respectively. A continuum-subtracted $\mathrm{H} \alpha$ map (Fig. 13b) from Papaderos et al. (2002) reveals an extended and complex morphology of the ionized gas emission in the upper half of the galaxy, where the $\mathrm{H} \alpha$ emission peaks, and shows a number of shells extending up to $\sim 0.5 \mathrm{kpc}$ NE. Another interesting feature is the extranuclear region $\mathrm{D}$, seen in the outer regions $\left(\mu \gtrsim 22 \mathrm{~J}\right.$ mag/ $\left.\square^{\prime \prime}\right)$ of IC 4662. Hidalgo-Gámez et al. (2001) find this $\mathrm{H} \alpha$ emitting region to be less metal-rich than the central SF regions (A), and to show a significant recession velocity difference $(250 \pm$ $150 \mathrm{~km} \mathrm{~s}^{-1}$, Hidalgo-Gámez et al. 2002) to IC 4662 . Our deep images show that region $\mathrm{D}$ is not well detached from the main body of diffuse $\mathrm{H} \alpha$ emission in IC4662 (contrary to HeydariMalayeri et al. 1990), but apparently connecting with regions A\&B through a chain of $\mathrm{H} \alpha$ sources.

Hidalgo-Gámez et al. (2002) have proposed that region D may be either a chemically and kinematically distinct complex within IC 4662, or a close companion object. The latter possibility is particularly interesting in view of the hypothesis that very close, gas-rich dwarf companions might be conceivable triggering agents of starburst activity in BCDs (Taylor et al. 1995; Pustilnik et al. 2001; Noeske et al. 2001).

From the available data, we resolve a wealth of morphological information in the central part (Fig. 13a) of IC 4662, notably the head-tail morphology of the starburst. Luminosities and colors of the brightest point sources in region A are in agreement with those of red supergiants, supporting the results by Heydari-Malayeri et al. (1990), as well as of blue supergiants and AGB stars (cf. the upper left inset in Fig. 13a; see Schulte-Ladbeck et al. 2001 for color limits separating the latter classes of giant stars).

As IC 4662 is located at low galactic latitude $\left(-17.8^{\circ}\right)$, photometric studies of its LSB component are complicated by the dense foreground Galactic stellar field. Also residuals in the removal of the NW bright star may affect the photometry for faint isophotal levels. A tentative exponential fit to the $J$ SBP yields for $R^{*}>65^{\prime \prime}$, i.e. outside significant nebular emission, a scale length of $\sim 0.21 \mathrm{kpc}$. However, inspection of the $J_{\text {LSB }}$ profile shows that the $\mathrm{BCD}$ follows a type $\mathrm{V}$ distribution, fitted best with Eq. (6) with a central surface brightness of $16.8 \mathrm{~J} \mathrm{mag} / \square$ ", a scale length of $\sim 150 \mathrm{pc}$ and a depression parameter as large as $q \approx 0.97$. If so, the starburst contributes $\sim 50 \%$ of the total $J$ light of the BCD. IC 4662 was hitherto classified as a dwarf irregular galaxy (cf. e.g. de Vaucouleurs et al. 1991). However, the structural properties of its LSB host, as well as its intense and spatially extended SF activity, place IC 4662 in the range of BCDs, making it probably one of the closest BCDs known.

\subsection{UM 488 (Mkn 1304, SBS 1139+006, UGC 6665)}

This is a distant $(D \approx 76 \mathrm{Mpc}$, Mirabel \& Sanders 1988) blue compact galaxy, known to be relatively metal-deficient $\left(Z_{\odot} / 8.5\right.$; Masegosa et al. 1994; Izotov \& Thuan 1999). Despite its resemblance (Fig. 14a) to cometary iI BCDs, UM 448 

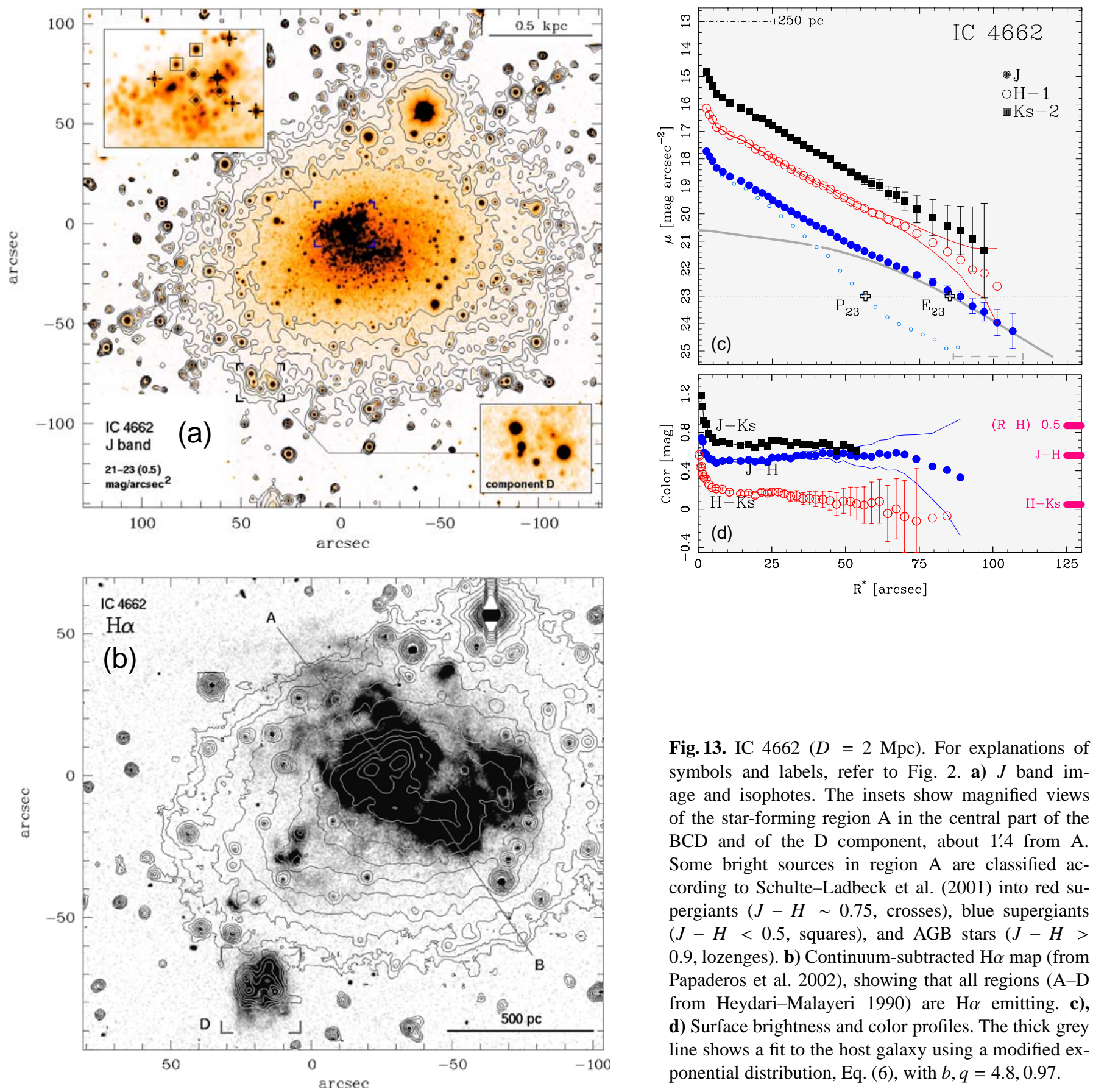

Fig. 13. IC $4662(D=2 \mathrm{Mpc})$. For explanations of symbols and labels, refer to Fig. 2. a) $J$ band image and isophotes. The insets show magnified views of the star-forming region $\mathrm{A}$ in the central part of the $\mathrm{BCD}$ and of the D component, about 1'.4 from A. Some bright sources in region A are classified according to Schulte-Ladbeck et al. (2001) into red supergiants $(J-H \sim 0.75$, crosses $)$, blue supergiants $(J-H<0.5$, squares), and AGB stars $(J-H>$ 0.9 , lozenges). b) Continuum-subtracted $\mathrm{H} \alpha$ map (from Papaderos et al. 2002), showing that all regions (A-D from Heydari-Malayeri 1990) are $\mathrm{H} \alpha$ emitting. c), d) Surface brightness and color profiles. The thick grey line shows a fit to the host galaxy using a modified exponential distribution, Eq. (6), with $b, q=4.8,0.97$.

does not qualify as a dwarf due to its intrinsic luminosity $\left(M_{B}=-19.7 \mathrm{mag}\right)$ and large linear extent (major axis length $\sim 25 \mathrm{kpc}$ ). Active star formation is taking place within an extended (diameter $\sim 7 \mathrm{kpc}$ ) high surface brightness region at the NE part of the galaxy, showing some fainter SW extension. The starburst component contributes $\approx 70 \%$ of the $J$ band light. Guseva et al. (2000) estimated the starburst luminosity to be powered by $1.6 \times 10^{6} \mathrm{O}$ stars (value referring to the distance assumed here), $0.6 \%$ of which are undergoing their Wolf-Rayet phase. Studies by Sage et al. (1992) indicate an ongoing SFR of $21 M_{\odot} \mathrm{yr}^{-1}$, and a large reservoir of molecular gas amounting to $2.8 \times 10^{9} M_{\odot}$ or $\sim 1 / 2$ of the H I mass $\left(5.8 \times 10^{9} M_{\odot}\right.$; Mirabel \& Sanders 1988) of UM 448.
On unsharp-masked images, the SF region splits into 3 high-surface brightness entities (see the inset in Fig. 14a), the brightest one being the reddest $\left(J-H^{*}=0.7 \mathrm{mag}\right)$ whereas the fainter regions $b$ and $c$ are slightly bluer $\left(J-H^{\ddagger} \approx 0.5 \mathrm{mag}\right)$. The SBPs (Fig. 14b) show an exponential decay for $R^{*}>15^{\prime \prime}$ with a $J$ band scale length of $3.8 \mathrm{kpc}$, somewhat smaller than the value $\sim 5.2 \mathrm{kpc}\left(14^{\prime \prime}\right)$ inferred in Telles et al. (1997).

\section{Discussion}

\subsection{The shape of NIR surface brightness profiles}

The SBPs derived for the sample BCDs in Sect. 4 bear close resemblance to those typically inferred from optical broad-band 

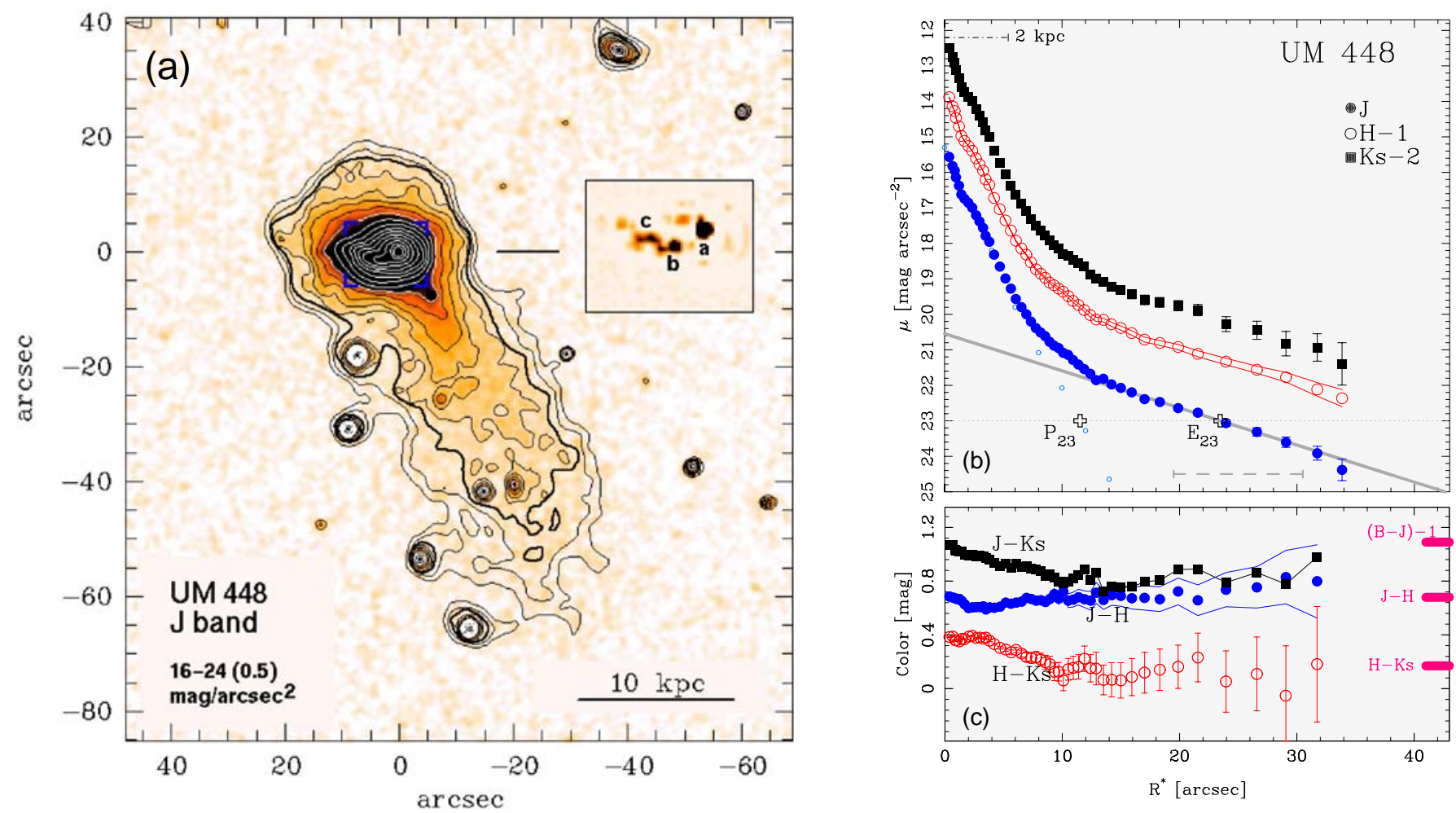

Fig. 14. UM $448(D=76.1 \mathrm{Mpc})$. For explanations of symbols and labels, refer to Fig. 2 . a) $J$ band image and isophotes. The inset shows a contrast-enhanced close-up of the star-forming regions (delimited by brackets in the main image); individual knots (a-c) are labeled. b), c) Surface brightness and color profiles.

data (cf., e.g., P96a, Telles et al. 1997; Marlowe et al. 1997; Doublier et al. 1997,1999; Cairós et al. 2001a). In most cases, SBPs show in their outer part an exponential intensity decrease and red, nearly constant colors. This outermost SBP part is attributable to the underlying LSB host galaxy which, except for a few rare cases, shows clear evidence for a several Gyr old stellar population. At intermediate and small radii the emission of a BCD is dominated by the younger stellar component. In the main class of $\mathrm{iE} \mathrm{BCDs} \mathrm{(LT86)} \mathrm{its} \mathrm{luminosity} \mathrm{output} \mathrm{is} \mathrm{re-}$ flected on two conspicuous SBP components.

1) At small radii $\left(R^{*} \lesssim 100 \mathrm{pc}\right.$ ), a feature commonly observed in optical and NIR SBPs is a central intensity excess. This is seen in e.g., the $J$ SBP of Tol 3, Mkn 178, Mkn 1329, Haro 14 (our sample) or Mkn 36 and UM 462 (C03). This component is due to the brightest and typically youngest stellar assembly. In most cases, this narrow innermost excess is due to one and the same region in both optical and NIR wavelengths. An exception is presented by Mkn 178, where the brightest region in the NIR is offset from that in the optical by $\sim 150 \mathrm{pc}$, or by the BCD Mkn 35 in the sample of $\mathrm{C} 03$.

2) The second SBP feature has been referred to as plateau in P96a. It shows a nearly constant or slowly decaying intensity, and extends typically out to $\mu \sim 24 B \mathrm{mag} / \square^{\prime \prime}$. SBPs with a prominent plateau on top a more extended exponential LSB envelope can in general not be adequately fit by a simple function (e.g. a Sérsic profile) over their whole intensity span. A meaningful decomposition of such SBPs is only possible when an extra component (e.g., a Sérsic distribution with $\eta \leq 1$ ) is introduced in order to fit the plateau (P96a, Cairós et al. 2001a). There is, so far, no observational support for the plateau being a dynamically distinct or even interaction-induced stellar entity with nearly constant $\mathcal{M} / \mathcal{L}$. Quite contrary, appreciable color gradients $\left(\lesssim 1.5 B-R\right.$ mag kpc $\left.{ }^{-1}\right)$ in the HSB part of BCDs indicate that the plateau light is mainly due to a young and moderately evolved stellar population. P96b have shown that a conspicuous plateau in the SBPs of many iE BCDs can naturally result from the superposition of diffuse and compact SF sources with varying luminosity and galactocentric distance on a more extended, exponential LSB component. Conversely, the plateau is nearly absent in the intrinsically brighter $\mathrm{nE} \mathrm{BCDs,} \mathrm{where}$ most of the starburst light originates from the nuclear region of the BCD. The overall SBPs of these systems show frequently a concave shape, fitted satisfactorily by Eq. (8) with a Sérsic exponent $\eta \geq 1$, and sometimes resemble a de Vaucouleurs profile.

Discerning the formation history of the stellar populations memorized in the plateau light poses a challenge for surface photometry and CMD studies, as nebular line emission (e.g. Tol 65; P99; Izotov et al. 2001, Tol 1214-277; F01) and patchy dust absorption (Tol 3, Mkn 178; this paper, Mkn 33 and Mkn 35; C03, I Zw 18; Cannon et al. 2002) may both hamper standard age-dating techniques. In addition, an important and mostly overlooked source of systematic uncertainties in the determination of colors or $E W \mathrm{~s}$ within the plateau stems from the unknown line-of-sight contribution of the underlying old LSB component. As pointed out in Sect. 3.3, the latter may 
redden colors of compact stellar clusters in the SF region by up to a few tenths of magnitude. Corrections of this order have also been reported for the iE BCDs Mkn 370 and Mkn 178 by Cairós et al. (2002) and Papaderos et al. (2002), respectively. Evidently, for such a correction one has to assume a model for the intensity distribution of the LSB component just beneath the $\mathrm{SF}$ regions (i.e., for $R^{*} \lesssim P_{23}^{\mathcal{J}}$ ). It is a common practice to extrapolate the exponential slope of the LSB periphery of BCDs all the way to $R^{*}=0^{\prime \prime}$. However, the universal validity of this procedure for BCDs has been questioned in, e.g., P96a. These authors discussed observational evidence for type $\mathrm{V}$ profiles in BCDs and proposed the alternative fitting formula Eq. (6) to approximate such convex distributions. Alternatively, C03 discuss cases where the LSB host galaxy shows a concave profile which is better fit by a Sérsic model with an exponent $\eta>1$. The photometric structure of the LSB host of BCDs will be discussed in the light of the present NIR data in the next section.

\subsection{Hints to a centrally flattening exponential distribution in the underlying stellar LSB component?}

Nine of the BCDs included in our sample (Table 2) show signatures of a type $\mathrm{V}$ profile in their LSB hosts. This is because an exponential law, or any Sérsic distribution with $\eta>1$, fitted to the outer part of their $J$ SBPs, predicts at intermediate to small radii a higher intensity than the observed value (cf. Sect. 3.2). Of course, pure exponential fits to $J_{\text {LSB }}$ profiles cannot always be definitely ruled out within the $1 \sigma$ uncertainties. However, such fits would either overestimate the central intensity of the stellar LSB host, hence underestimate the luminosity fraction of the SF component (see below) or systematically overestimate the $J$ band exponential scale length, thus imply an implausibly large $B-J$ color gradient for the old underlying LSB population.

The limited size of our present sample does hardly allow to estimate the frequency of type $\mathrm{V}$ profiles in BCDs. This issue will be addressed in a forthcoming paper of this series, focussing on the complete NIR sample. However, the evidence gathered so far (see also Sect. 3.2) strongly supports the idea that a substantial fraction of BCDs shows type $V$ profiles in its LSB host galaxy. The rare detection of type $\mathrm{V}$ profiles in BCDs in previous optical studies can be explained by the fact that in those wavelengths extended starburst emission overshines the LSB host within typically its inner $\sim 2$ exponential scale lengths (P96b, Noeske 1999; Cairós 2000; Cairós et al. 2001a). As a result, a type $\mathrm{V}$ profile in $\mathrm{iE} / \mathrm{nE}$ BCDs could be detected only when a significant depression of the exponential LSB slope occurs for $R^{*}>2 \alpha$, i.e. if $b>2$ in Eq. (6). NIR observations do not overcome, but alleviate the problem of severe light pollution by the burst, proving in many cases crucial for disentangling a pure exponential from a type $\mathrm{V}$ distribution in the underlying LSB galaxy.

This is illustrated in Fig. 15 on the example of the iE BCD Haro 14. Its $B \mathrm{SBP}$ is roughly exponential out to $R^{*}=40^{\prime \prime}(\mu \sim$ $\left.27 B \mathrm{mag} / \square^{\prime \prime}\right)$, suggesting that the exponential slope seen in the LSB periphery continues all the way to $R^{*}=0^{\prime \prime}$. In that case,

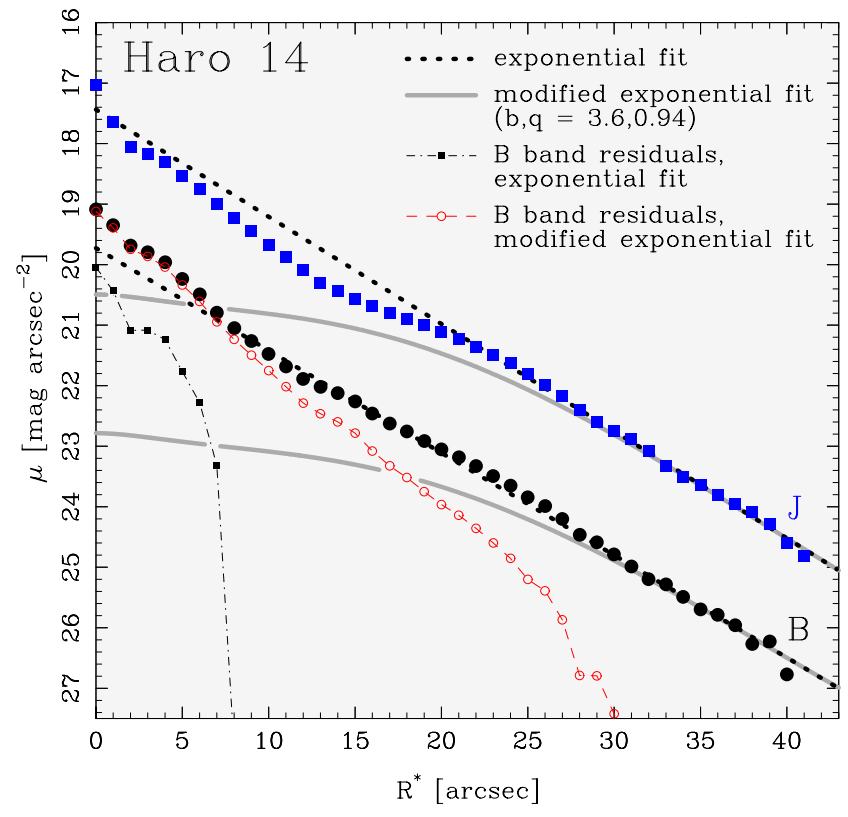

Fig. 15. Decomposition of the $B$ band SBP of Haro 14 (filled circles) assuming for its LSB host a pure exponential model (dotted line) or a modified exponential distribution (Eq. (6); thick gray curve) with the flattening parameters $(b, q)=(3.6,0.9)$ derived in the $J$ band. The surface brightness distribution of the star-forming component, as obtained by subtraction of the respective LSB model from the $B$ SBP is illustrated by the filled and open interconnected symbols (see discussion in the text).

one is tempted to conclude that the ongoing burst gives rise to a modest luminosity increase, only. Indeed, subtraction of the exponential LSB model from the SBP suggests that SF sources (small interconnected squares in Fig. 15) are all confined within $R^{*}=8^{\prime \prime}$ and that they account for no more than $7 \%(\geq 16.5 \mathrm{mag})$ of the $B$ light of the BCD. Evidently, this conclusion is hardly compatible with the extended morphology of the SF component and copious $\mathrm{H} \alpha$ emission of Haro 14 (cf. Marlowe et al. 1997; Doublier et al. 1999).

One arrives at a quite opposite conclusion on inspection of the NIR profiles. Figure 15 shows that a pure exponential law falls short of fitting the $J$ SBP inwards of $R^{*}<20^{\prime \prime}$; a plausible fit to the NIR data must invoke a significant flattening of the exponential slope inside $\gtrsim 3$ scale lengths of the LSB component. An adequate fit to the $J_{\mathrm{LSB}}$ is best achieved with a med (Eq. (6)) with $(b, q)=3.6,0.94$. Such a strongly flattened exponential LSB model yields a stellar mass by a factor $\sim 3$ smaller than that of a purely exponential profile and implies that the starburst (small interconnected circles in Fig. 15) contributes $\sim 60 \%$ of the $B$ light within the Holmberg radius, i.e. more than a factor of eight larger than the value inferred before. The isophotal $B$ magnitude within $25 B$ mag/ $\square^{\prime \prime}$ is then increased by $\sim 1.1 \mathrm{mag}$ due to the starburst. This value is still compatible to the mean value of 0.75 mag deduced for BCDs in P96b and Salzer \& Norton (1999).

The considerations above show that the choice of the model for the LSB emission may have far-reaching implications to our view about the SF amplitude and photometric fading of BCDs. 
The usual assumption of a purely exponential LSB model may, in some cases, strongly underestimate the luminosity fraction of the young stellar population, and lead to the conclusion that a "burst" is merely a minor luminosity enhancement in the lifetime of a BCD. If, on the contrary, the LSB emission is assumed to follow a type $\mathrm{V}$ profile, then the estimated luminosity of the superimposed young stellar population may increase by more than a magnitude.

Consequently, different LSB models imply different amounts of photometric fading of a BCD once SF activities have terminated. This has to be borne in mind when discussing evolutionary links between BCDs and other dwarf galaxies, as well as a possible fading of luminous blue compact galaxies at medium redshift to local spheroidals (cf. e.g. Guzmán et al. 1998). Note that the assumption of a type $V$ profile has no effect on the extrapolated central surface brightness $\mu_{\mathrm{E}, 0}$ and the exponential scale length $\alpha$ of the LSB host galaxy of a BCD. As a result it is not expected to significantly change the systematic difference between BCDs and other types of dwarf galaxies on the $\mu_{\mathrm{E}, 0}-M_{\mathrm{LSB}}$ and $\log (\alpha)-M_{\mathrm{LSB}}$ parameter space (P96b, Marlowe et al. 1997; Salzer \& Norton 1999; Papaderos et al. 2002).

A dedicated investigation of the frequency and origin of systematic deviations from the exponential law (e.g. a type V profile, or Sérsic profile with $\eta>1$ ) in the stellar LSB host of BCDs is apparently of great interest. A first step towards such studies is to test the suitability of different empirical functions in approximating the observed LSB intensity distribution. This issue will be briefly discussed in the following section.

\subsection{The Sérsic law vs. the med for studies of type $V$ SBPS}

As noted in Sect. 3.2, also the Sérsic law, commonly applied to structurally analyze various extragalactic systems, can approximate type V SBPs. In this section, we further discuss why in this work preference was given to the med model to fit and quantitatively study such SBPs.

Both the Sérsic law and the med are fitting functions of empirical origin (Sect. 3.2). The choice of either model does therefore not imply any assumptions on the physical background of type V SBPs, such as the dynamics or stellar mass distribution of a galaxy. However, although either function was found to satisfactorily approximate the type V SBPs found in this work, they principally differ with respect to their shape. This difference is evident in the comparison shown in Fig. 16, where we fit for illustrative purposes a Sérsic profile (thin dashed line) to the med model (heavy line, labelled 4) obtained for the LSB component of Haro 14 in Sect. 4. Distributions 2 and 3 illustrate med profiles with equal extrapolated central surface brightness $\mu_{\mathrm{E}, 0}\left(17.46 \mathrm{~J} \mathrm{mag} / \square^{\prime \prime}\right)$ and exponential scale length $\alpha(0.37 \mathrm{kpc})$ as 4 , but differing degrees of flattening $(q)$ with respect to the pure exponential $(q=0)$ model 1 .

The med 4 compares best to a Sérsic profile with a central surface brightness $\mu_{\mathrm{S}, 0}=20.45 \mathrm{~J} \mathrm{mag} / \square^{\prime \prime}$, a scale length $\beta \approx 1.14 \mathrm{kpc}$ and an exponent $\eta=0.57$. Note that the
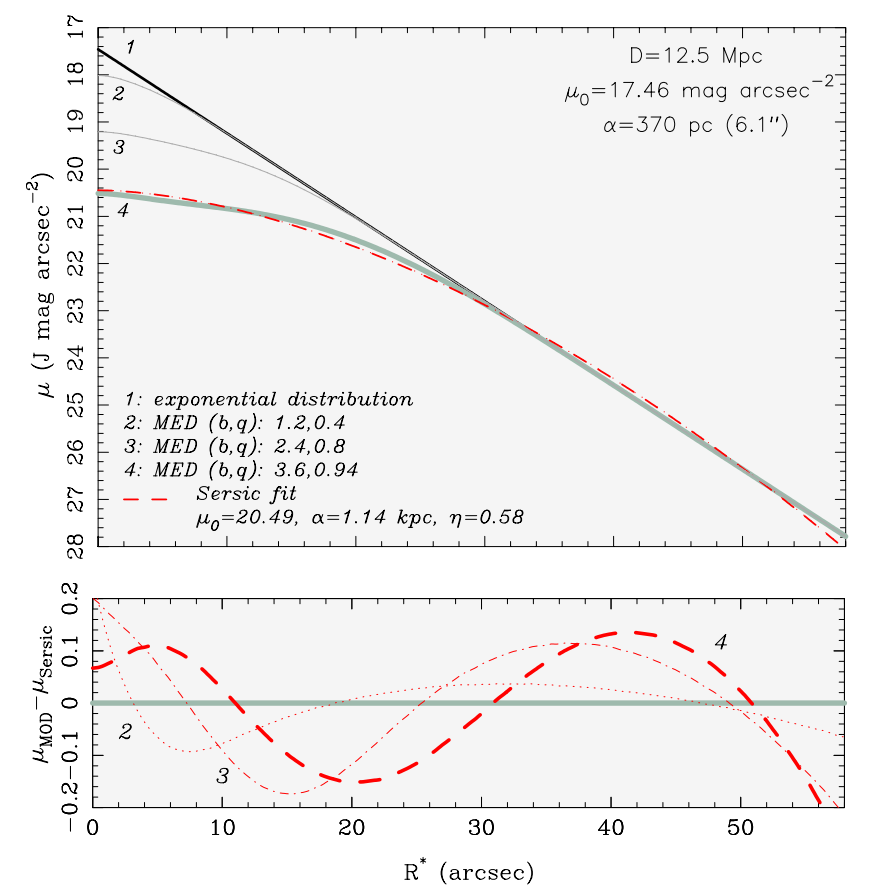

Fig. 16. Upper panel: Fit to the LSB component of Haro 14 (thick gray curve, labelled 4) assuming an inwards flattened exponential distribution (med, Eq. (6)) with the extrapolated central surface brightness $\mu_{\mathrm{E}, 0}$ and exponential scale length $\alpha$ indicated at the upper-right, and flattening parameters $(b, q)=(3.6,0.94)$. Distributions 2 and 3 correspond to a med with equal $\mu_{\mathrm{E}, 0}$ and $\alpha$ but different $(b, q)$, and 1 describes a pure exponential distribution $(q=0)$. The dashed curve shows a fit to distribution 4 using a Sérsic fitting law (Eq. (8)). Lower panel: Residuals between the assumed med distributions 2 through 4 (upper panel) and Sérsic fits.

deduced $\mu_{\mathrm{S}, 0}$ compares well to the actual central surface brightness of the LSB host galaxy

$\mu_{\mathrm{E}, 0}+2.5 \log \left(\frac{1}{1-q}\right) \approx 20.5 \mathrm{mag} / \square^{\prime \prime}$,

implied by the med model.

It is evident that the Sérsic law and the med distribution in Fig. 16 closely follow each other over a radius range of nearly 10 scale lengths $\alpha$. Nevertheless, the lower panel of Fig. 16 shows that the residuals between either model are small ( $\$ 0.2 \mathrm{mag}$ ), but systematic. This difference reflects the fact that a Sérsic law with $\eta<1$ differs from an exponential at all radii, while the med approaches an exponential slope for $R>b \alpha$.

We recall that both a Sérsic and a med model were found to approximate well the inner portion of a med profile (e.g. within $\sim 1.5$ cutoff-radii $b \alpha$ ). The question which distribution gives a more adequate description of the observed type V SBPs can hence only be assessed through studies of the outer parts of such profiles. If the type V SBPs show a perfectly exponential fall-off in their outer parts, continuing several scale lengths beyond a cutoff radius $b \alpha$ (cf. Eq. (6)), a Sérsic law will fail to correctly reproduce these light distributions, and no Sérsic model fit to the LSB emission can correctly recover the exponential scale length $\alpha$ and will not be free of ambiguity 
(Sect. 5.3.1). Alternatively, if subsequent studies show that the LSB profiles deviate systematically from the exponential law at all radii, then a Sérsic profile might be the preferable fitting law, albeit the drawbacks discussed in the following Sect. 5.3.1.

Such considerations call for deeper photometric studies of the LSB component of BCDs, and in dwarf galaxies in general. An ultimate check requires deep surface photometry, allowing to derive SBPs out to $\sim 10 \alpha$ with an accuracy better than 0.2 mag (see the residuals in Fig. 16), not reached by currently available data.

As long as tests of this kind await to be done, we argue, in agreement with $\mathrm{C} 03$, that a pure (Eq. (5)) or modified exponential (Eq. (6)) fitting formula should be preferably used to fit the underlying LSB component of BCDs. Previous optical studies (e.g. LT86, P96a, Telles et al. 1997; Marlowe et al. 1997; Salzer \& Norton 1999; Vennik et al. 2000; Cairós et al. 2001a, 2001b; Makarova et al. 2002) of BCDs suggest that those systems mostly show no strong deviations from an exponential law over their outer LSB SBPs. Also if small deviations from an exponential law which are hardly detectable in current data should exist, a med gives a robust approximation by fixing the LSB profile shape. While a Sérsic law at first view offers a greater flexibility in this profile region, its application in the extremely low $S / N$ regime to quantify marginal deviations from an exponential is problematic, as discussed in the following section.

\subsubsection{A cautionary note on structural analyses of BCDs using the Sérsic law}

A drawback of Sérsic models when fitted to type $\mathrm{V}$ profiles is that the solution can vary considerably, depending on the fitted radius range. For instance, a Sérsic fit to the med 4 (Fig. 16) for $<23.5 J \mathrm{mag} / \square^{\prime \prime}$, or within $5 \alpha\left(\sim 30^{\prime \prime}\right)$ yields an $\eta=0.45$, significantly lower than the value above. Note that a Sérsic exponent $\eta<0.5$ reflects a central minimum in the radial luminosity density distribution of the LSB component (P96a). The stability of the shape parameter $\eta$ has been explored in detail in $\mathrm{C} 03$; these authors remarked that a Sérsic fit can be very uncertain for concave SBPs, where the exponent $\eta$ can vary by up to an order of magnitude, depending on the fitted range and the radial sampling of points in the profile.

Another objection to the view that Sérsic models offer a robust tool to systematize the structural properties of dwarfs comes from the degeneracy of the shape parameter $\eta$ vs. the pseudo-scale length $\beta$. A fit of Eq. (8) to the med distribution 2 yields an $\eta=0.94$ and a $\beta \approx 0.44 \mathrm{kpc}(>\alpha)$. As for profile 3 , it is best approximated with an $\eta=0.75$ and $\beta \approx 0.72 \mathrm{kpc}(2 \alpha)$. We see that while the Sérsic exponent $\eta$ decreases with increasing degree of flattening, i.e. as we go from distribution 2 towards 4 , the corresponding scale length $\beta$ increases from $0.44 \mathrm{kpc}$ to $1.15 \mathrm{kpc}$. Actually, the exponential scale length $\alpha$ of the med profiles considered here is nowhere recovered by fitting a Sérsic model. Instead, one is left with the pseudo-scale length $\beta$, which by itself alone carries no quantitative information on the structural properties of the LSB component.
Obviously, the scale length $\beta$ is only meaningful in connection with $\eta$. However, these two quantities are rendered impractical for a systematic study of the LSB component by their strong non-linear coupling (see discussion in e.g. Young \& Currie 1994; Cellone \& Buzzoni 2001), and dependence on the fitting procedure (see above). Furthermore, observational uncertainties connected with, e.g., the sky subtraction (see e.g. Cellone \& Buzzoni 2001, C03), filtering of images prior to surface photometry, and imperfect removal of background sources may also skew the $\beta$ vs. $\eta$ parameters in a hardly predictable manner, making Sérsic fits to the outer part of SBPs a hazardous procedure $(\mathrm{C} 03)$. These problems are probably not worrisome in studies of early type galaxies, i.e. systems with little morphological distortions and a nearly constant $\mathcal{M} / \mathcal{L}$, where also the central, high $S / N$ regions are accessible to constrain the global photometric structure. In irregular SF galaxies, however, the derived Sérsic parameters depend on subjective choices in the data processing (see above), profile fitting and, equally important, on the profile extraction methods themselves. This is particularly important in the case of BCDs, where mostly only the outer, low $S / N$ part of the LSB host is accessible to structural studies.

It is conceivable that the latter circumstances imprint the Sérsic parameters $\left(\mu_{\mathrm{S}, 0}, \beta, \eta\right)$ inferred recently for a sample of luminous BCGs by Bergvall \& Östlin (2002). We believe that the extremely large $\eta_{\text {LSB }}$ exponents they derive (up to 20), in conjunction with central surface brightnesses $\ll 0 \mathrm{mag} / \mathrm{arcsec}^{2}$, should be regarded formal only, rather than a manifestation of an extraordinarily dense Dark Matter halo which, as advocated by these authors, dominates the stellar dynamics of a BCD.

\subsection{The colors of the stellar LSB host galaxy}

The available NIR data allowed not only for the detection and structural analysis of the LSB component for all sample BCDs, but also for the determination of at least one NIR color in the LSB host (Sect. 3.5).

The $B-J$ vs. $J-H$ and $H-K_{\mathrm{s}}$ vs. $J-H$ LSB colors (Table 3), whenever available, are displayed in Fig. 17. For comparison, color predictions from the GALEV evolutionary synthesis model (Schulz et al. 2002; Anders, Bicker \& Fritze - v. Alvensleben 2002, priv. comm.) are overlaid in both panels, calculated for stellar populations with metallicities of $Z_{\odot} / 50$ and $Z_{\odot} / 2.5$, formed either in an instantaneous burst or continuously with a constant SFR. As evident from Fig. 17, NIR colors $\left(J-H\right.$ vs. $\left.H-K_{\mathrm{s}}\right)$ show little evolution for old stellar populations ( $\leqslant 0.1 \mathrm{mag}$ for an age $>0.5 \mathrm{Gyr}$ ). However, optical-NIR colors, such as, e.g., $B-J$ (right panel of Fig. 17), change by up to $\sim 1.5$ mag for ages $>0.5 \mathrm{Gyr}$. Within the above discussed errors, such colors will allow to constrain the age of the stellar LSB host of old $\mathrm{iE} / \mathrm{nE}$ BCDs with a precision of 1-2 Gyr, once precise spectroscopic measurements of its metallicity become available (see the discussion of Gil de Paz et al. 2000b). Figure 17 shows that the observed colors are generally compatible with the GALEV predictions within their $1 \sigma$ uncertainties. This is also the case for model predictions from PEGASE (Fioc \& Rocca-Volmerange 1997). 

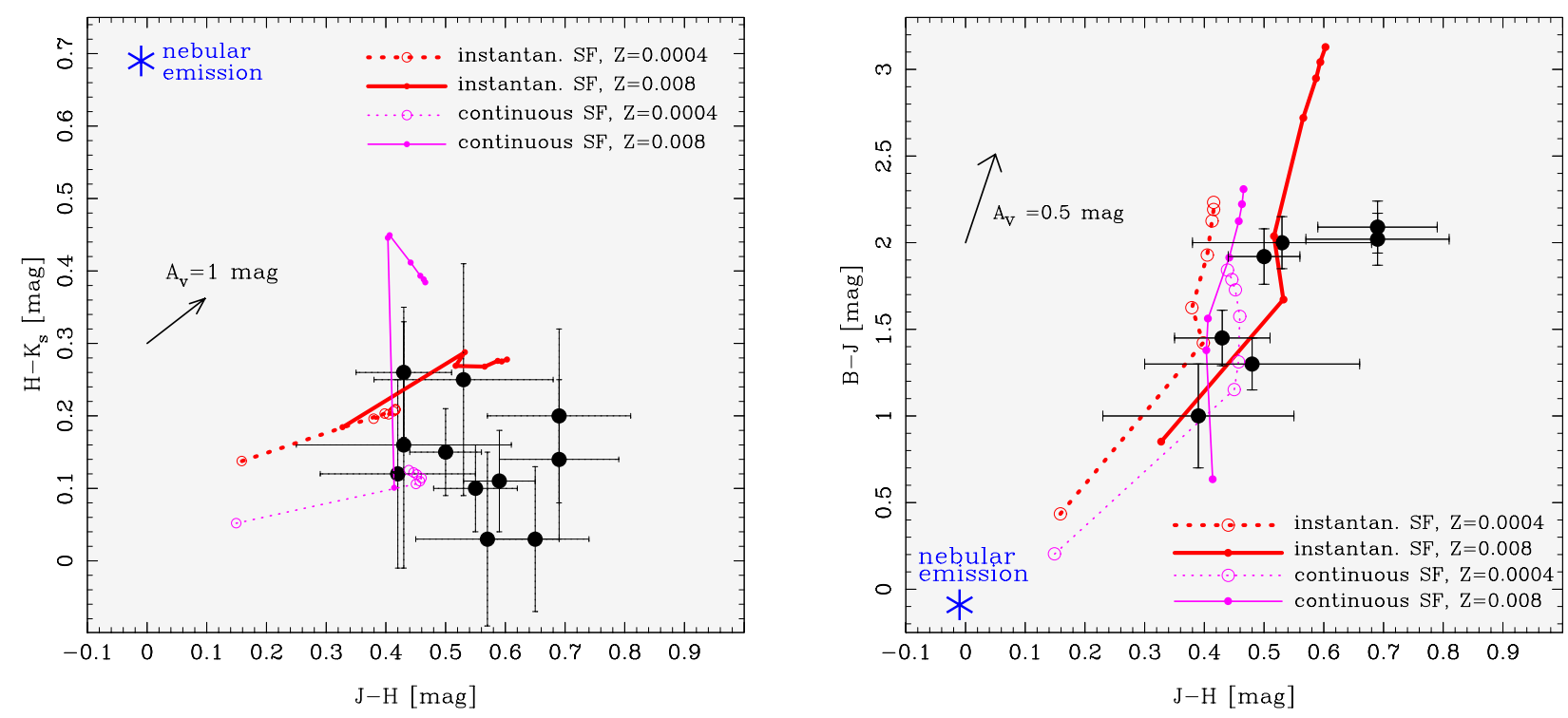

Fig. 17. Colors of the stellar LSB host galaxies (filled circles) as listed in Table 3. For comparison, we show the color evolution of synthetic stellar populations, calculated with the GALEV evolutionary synthesis model (Schulz et al. 2002; Anders et al. 2002, priv. comm.). An initial mass function with a Salpeter slope and respective lower and upper stellar mass cutoffs of 0.08 and $100 \mathrm{M}_{\odot}$ was assumed. We show single-age stellar populations, formed in an instantaneous burst, as well as in a continuous process with a constant SFR, both calculated for metallicities of $Z_{\odot} / 50$ and $Z_{\odot} / 2.5$. For clarity, only population ages of $0.1,0.5,1,3,7,10$ and $14 \mathrm{Gyr}$ are shown. The youngest $(0.1 \mathrm{Gyr})$ point of each evolutionary track corresponds to the bluest $H-K_{\mathrm{s}}$ and $B-J$ color. All NIR colors shown are transformed to the 2MASS photometric system (see Sect. 2.4.1), while the $B$ band refers to the Johnson system. Note that the error bars indicate cumulative uncertainties due to all major error sources (see Sect. 3.5). The colors of ionized gas emission from a metal-deficient $\left(Z_{\odot} / 20\right) \mathrm{H}$ II region $\left(T_{\mathrm{e}}=10^{4} \mathrm{~K}, n_{\mathrm{e}}=100 \mathrm{~cm}^{-3}\right.$, Case B recombination; cf. Krüger 1992) are indicated by the asterisk. Arrows show reddening vectors.

Five BCDs in our sample, Pox 4, Pox 4B, Tol 1400-411, Tol 65 and Tol 1214-277 (Table 3), show blue $(J-H<$ $0.5, B-J<1.5)$ colors in their LSB component. This may be partly due to an appreciable contribution of extended nebular emission, as suggested by previous optical spectrophotometric work. The blue optical-NIR LSB colors of Tol 65 and Tol 1214-277 may be attributed to a comparatively young ( $\$ 1 \mathrm{Gyr}$ ) photometrically dominant stellar population (cf. P99, F01). The optical-NIR colors of the LSB host of the remaining sample BCDs can be reconciled with an old stellar population of subsolar metallicity. This result is in line with most published optical-NIR photometry on stellar hosts of BCDs (Gil de Paz et al. 2000b; Vanzi et al. 2002; Papaderos et al. 2002), which indicates a comparable range of colors for the LSB population of BCDs.

We can compare the NIR colors of the LSB component with data from the literature for three sample BCDs, only (Tol 3, Haro 14 and UM 461). The colors of Tol 3 compare well with those reported by Vanzi et al. (2002) over the whole radius range of $\left(R^{*}=0^{\prime \prime} \ldots 50^{\prime \prime}\right)$. Our photometry for Tol 3 and Haro 14 is also in agreement with Doublier et al. (2001) for small radii (for $R^{*} \lesssim 15^{\prime \prime}$ and $R^{*} \lesssim 10^{\prime \prime}$, respectively). For larger radii, however, the NIR colors by Doublier et al. are not compatible with our results within the $1 \sigma$ uncertainties. Outside the star-forming component the color profiles by Doublier et al. approach values of $J-H>2$ for Tol 3 , and $J-H \approx 1.0 \ldots>2.5$, $H-K \approx 0 \ldots-0.6$ for Haro 14 . For UM 461, their color profiles vary between $\approx 1.1 \ldots 2.3(J-H)$ and $\approx-0.6 \ldots 0.5(H-K)$.

\section{Summary and conclusions}

We have analyzed deep Near-Infrared (NIR) $J, H, K$ images of 12 Blue Compact Dwarf (BCD) Galaxies and one luminous Blue Compact Galaxy. These objects, together with those studied in an accompanying paper (Cairós et al. 2003), constitute the first part of a sample of 40 BCDs for which deep NIR images were obtained in the framework of a large-scale multiwavelength study. The limiting surface brightnesses of our data, $\sim 23.5$ to $25.5 \mathrm{mag} / \square^{\prime \prime}$ in $J$ and $\sim 22$ to $24 \mathrm{mag} / \square^{\prime \prime}$ in $K$, allow for the detection and study of the NIR structural properties and colors of the underlying stellar low-surface brightness (LSB) component in all sample galaxies. This evolved LSB host, underlying the star-forming regions is known to exist in the majority of BCDs and to contain the bulk of the stellar mass in these systems. Consequently, a systematic determination of its structural properties (e.g., radial stellar surface density distribution, central surface brightness, exponential scale length) and of the gravitational potential it forms may prove crucial for the understanding of the starburst activity, dynamics and evolution of BCDs. Other than in optical wavelengths, where extended starburst emission hides the LSB host inside its inner 2-3 exponential scale lengths, NIR studies allow to extend surface photometry of the underlying old stellar background to a smaller galactocentric distance, thereby better constrain its overall intensity distribution. Our results can be summarized as follows:

1. Surface brightness profiles (SBPs) of our sample BCDs can at large galactocentric radii $R^{*}$ be well approximated 
by an exponential fitting law, in agreement with previous observational evidence based on deep optical surface photometry. Also, the exponential scale lengths derived in the optical and NIR spectral domain are in mutual agreement, implying minor optical-NIR color gradients in the LSB component.

2. The LSB host galaxy of several sample BCDs shows on a galactocentric radius comparable to the size of the starforming component (within its inner 1-3 exponential scale lengths) a conspicuous intensity depression with respect to the purely exponential slope, observed for larger radii. This type of an inwards flattening exponential SBP, classified type V in Binggeli \& Cameron (1991), has been frequently derived in dwarf irregular and dwarf elliptical galaxies. The rare detection of type $\mathrm{V}$ profiles in BCDs from previous optical studies can be attributed to the fact that in those wavelengths extended starburst emission overshines the underlying LSB population within typically its inner $\sim 2$ exponential scale lengths. A possible high frequency of type $\mathrm{V}$ profiles among dwarf galaxies will not have a notable effect on the structural difference between BCDs and dwarf irregulars on the parameter space defined by the central surface brightness, exponential scale length and absolute magnitude of their LSB host galaxy. However, it may have important implications for our view about BCDs, as it would significantly increase the estimated starburst-to-LSB luminosity fraction, and therefore the amount of photometric fading of these systems, once the starburst activity has terminated. This information is crucial for, e.g., establishing or discarding the hypothesis of faint dwarf spheroidals being the evolutionary endpoints of BCDs. In the same way, a type $\mathrm{V}$ intensity distribution would impose new observational constraints to the derivation of the total stellar mass and its radial density distribution within the underlying LSB host galaxy of BCDs.

3. The exact shape and physical origin of type V SBPs in dwarf galaxies are still to be investigated. We find that such SBPs can be well approximated by a modified exponential fitting formula proposed in Papaderos et al. (1996a). Alternatively, a Sérsic law can also yield good fits to type V profiles, albeit small systematic residuals. However, the practical applicability of the Sérsic law to the LSB emission of BCDs is limited by the strong non-linear coupling of its free parameters, and the extreme sensitivity of the achieved solutions to, e.g., small uncertainties in the sky subtraction and SBP derivation.

4. For the majority of the LSB host galaxies in our sample we derive optical-NIR colors indicative of an evolved stellar population of subsolar metallicity. The metal-deficient BCDs Tol 65 and Tol 1214-277 show blue colors $(B-J<$ $1.3 \mathrm{mag}$ ) in their underlying stellar LSB component.

5. NIR images, in combination with optical data reveal for some BCDs signatures of appreciable and non-uniform dust absorption on a spatial scale as large as $\sim 1 \mathrm{kpc}$. The NIR images, being less affected by dust extinction and widespread ionized gas emission than optical data, allow for the detection of a variety of morphological features within the star-forming component, e.g. coherent assemblies of compact stellar clusters, that might be remnants from previous episodes of collective star formation. An elaborate multiwavelength investigation of such sources holds the promise of a better understanding of the history and spatial progression of star-forming activity in BCDs.

Acknowledgements. Research by K.G.N. has been supported by the Deutsche Forschungsgemeinschaft (DFG) Grants FR325/50-1 and FR325/50-2. LMC acknowledges support from the European Community Marie Curie Grant HPMF-CT-2000-00774. We thank U. Fritze - v. Alvensleben, P. Anders, J. Bicker and J. Schulz for kindly providing the GALEV models. This research has made use of the NASA/IPAC Extragalactic Database (NED) which is operated by the Jet Propulsion Laboratory, CALTECH, under contract with the National Aeronautic and Space Administration. This publication makes use of data products from the Two Micron All Sky Survey, which is a joint project of the University of Massachusetts and the Infrared Processing and Analysis Center/California Institute of Technology, funded by the National Aeronautics and Space Administration and the National Science Foundation.

\section{References}

Alton, P. B., Draper, P. W., Gledhill, T. M., et al. 1994, MNRAS, 270 , 238

Andredakis, Y. C., Peletier, R. F., \& Balcells, M. 1995, MNRAS, 275, 874

Andreon, S. 2002, A\&A, 382, 821

Babul, A., \& Rees, M. J. 1992, MNRAS, 255, 346

Beck, S. C., Kelly, D. M., \& Lacy, J. H. 1997, AJ, 114, 585

Bergvall, N., \& Östlin, G. 2002, A\&A, 390, 891

Bertelli, G., Bressan, A., Chiosi, C., Fagotto, F., \& Nasi, E. 1994, A\&AS, 106, 275

Binggeli, B., \& Cameron, L. M. 1991, A\&A, 252, 27

Binggeli, B., \& Jerjen, H. 1998, A\&A, 333, 17

Cairós, L. M. 2000, Ph.D. Thesis, Instituto de Astrofísica de Canarias

Cairós, L. M., Vílchez, J. M., González Pérez, J., Iglesias-Páramo, J., \& Caon, N. 2001a, ApJS, 133, 321

Cairós, L. M., Caon, N., Vílchez, J. M., González-Pérez, J., \& Muñoz-Tuñón, C. 2001b, ApJS, 136, 393

Cairós, L. M., Caon, N., García-Lorenzo, B., Vílchez, J. M., \& Muñoz-Tuñón, C. 2002, ApJ, 577, 164

Cairós, L. M., Caon, N., Papaderos, P., et al. 2003, ApJ, in press (C03)

Campbell, A. W., \& Terlevich, R. 1984, MNRAS, 211, 15

Cannon, J. M., Skillman, E. D., Garnett, D. R., \& Dufour, R. J. 2002, ApJ, 565, 931

Caon, N., Capaccioli, M., \& D’Onofrio, M. 1993, MNRAS, 265, 1013

Cardelli, J. A., Clayton, G. C., \& Mathis, J. S. 1989, ApJ, 345, 245

Carpenter, J. M. 2001, AJ, 121, 2851

Cellone, S. A., \& Buzzoni, A. 2001, A\&A, 369, 742

Cellone, S. A., Forte, J. C., \& Geisler, D. 1994, ApJS, 93, 397

Conti, P. S., \& Vacca, W. D. 1994, ApJ, 423, L97

Corbin, M. R., Korista, K. T., \& Vacca, W. D. 1993, AJ, 105, 1313

Cutri, R. M., Skrutskie, M. F., Van Dyk, S. et al. 2000, The 2MASS Explanatory Supplement

Davidge, T. J. 2002, PASP, 114, 1206

Davies, J. I., \& Phillipps, S. 1988, MNRAS, 233, 553

Dekel, A., \& Silk, J. 1986, ApJ, 303, 39 
de Vaucouleurs, G., de Vaucouleurs, A., Corwin, H. G., et al. 1991, Third Reference Catalogue of bright Galaxies (Springer Verlag)

Doublier, V., Comte, G., Petrosian, A., Surace, C., \& Turatto, M. 1997, A\&AS, 124, 405

Doublier, V., Caulet, A., \& Comte, G. 1999, A\&AS, 138, 213

Doublier, V., Caulet, A., \& Comte, G. 2001, A\&A, 367, 33

Duc, P.-A., Papaderos, P., Balkowski, C., et al. 1999, A\&AS, 136, 539

Elias, J. H., Frogel, J. A., \& Humphreys, R. M. 1985, ApJS, 57, 91

Ferrara, A., \& Tolstoy, E. 2000, MNRAS, 313, 291

Fioc, M., \& Rocca-Volmerange, B. 1997, A\&A, 326, 950

Freeman, K. C. 1970, ApJ, 160, 811

Fricke, K. J., Izotov, Y. I., Papaderos, P., Guseva, N. G., \& Thuan, T. X. 2001, AJ, 121, 169 (F01)

Fritz, T. 2000, Ph.D. Thesis, University of Bonn

Fujita, A., Mac Low, M.-M., Meiksin, A., \& Ferrara, A. 2001, Am. Astron. Soc. Meet., 198, 5703

Gallagher, J. S. III., \& Hunter, D. A. 1989, AJ, 98, 806

García, A. M. 1993, A\&AS, 100, 47

Gil de Paz, A., Zamorano, J., Gallego, J., \& Domínguez, F. de B. 2000a, A\&AS, 145, 377

Gil de Paz, A., Zamorano, J., \& Gallego, J. 2000b, A\&A, 361, 465

González-Riestra, R., Rego, M., \& Zamorano, J. 1987, A\&A, 202, 72

Graham, A., Lauer, T. R., Colless, M., \& Postman, M. 1996, ApJ, 465, 534

Guseva, N. G., Izotov, Y. I., Papaderos, P., et al. 2001, A\&A, 378, 756

Guseva, N. G., Izotov, Y. I., \& Thuan, T. X. 2000, ApJ, 531, 776

Guzmán, R., Jangren, A., Koo, D. C., Bershady, M. A., \& Simard, L. 1998, ApJ, 495, L13

Hensler, G., Dickow, R., \& Junkes, N. 1997, RMxAA Conf. Ser., 6, 90

Heydari-Malayeri, M., Melnick, J. \& Martin, J.-M. 1990, A\&A, 234, 99

Hidalgo-Gámez, A. M., Masegosa, J., \& Olofsson, K. 2001, A\&A, 367,388

Hidalgo-Gámez, A. M., Masegosa, J., \& Olofsson, K. 2002, RMxAA Conf. Ser., 12, 242

Hirashita, H., Tamura, N., \& Takeuchi, T. T. 2000, IAU Symp., 201, 64

Hunt, L. K., Thuan, T. X., \& Izotov, Y. I. 2003, ApJ, 588, 281

Hunter, D., \& Hoffman, L. 1999, AJ, 117, 2789

Izotov, Y. I., \& Thuan, T. X. 1999, ApJ, 511, 639

Izotov, Y. I., \& Thuan, T. X. 2002, ApJ, 567, 875

Izotov, Y. I., Thuan, T. X., \& Lipovetsky, V. A. 1997, ApJS, 108, 1

Izotov, Y. I., Chaffee, F. H., \& Green, R. F. 2001, ApJ, 562, 727

James, P. A. 1994, MNRAS, 269, 176

Jarrett, T. H., Chester, T., Cutri, R., Schneider, S., \& Huchra, J. 2000, AJ, 119, 2498

Johnson, K. E., Leitherer, C., Vacca, W. D., \& Conti, P. S. 2000, AJ, 120,1273

Karachentsev, I. D., Sharina, M. E., Dolphin, A. E., et al. 2002, A\&A, 385,21

Kobulnicky, H. A., Kennicutt, R. C., \& Pizagno, J. L. 1999, ApJ, 514, 544

Krüger, H. 1992, Ph.D. Thesis, University of Göttingen

Krüger, H., Fritze-v. Alvensleben, U., \& Loose, H.-H. 1995, A\&A, 303,41

Kunth, D., \& Sargent, W. L. W. 1981, A\&A, 101, L5

Kunth, D., \& Sargent, W. L. W. 1983, ApJ, 273, 81

Kunth, D., Maurogordato, S., \& Vigroux, L. 1988, A\&A, 204, 10

Lin, D. N. C., \& Faber, S. M. 1983, ApJ, 266, L21

Loose, H. H., \& Thuan, T. X. 1986, Star Forming Dwarf Galaxies and Related Objects, 73 (LT86)

Lowenthal, J. D., Koo, D. C., Guzman, R., et al. 1997, ApJ, 481, 673
MacArthur, L. A., Courteau, S., \& Holtzman, J. A. 2003, ApJ, 582, 689

Makarova, L., Karachentsev, I., Takalo, L. O., Heinaemaeki, P., \& Valtonen, M. 1998, A\&AS, 128, 459

Makarova, L. N., Karachentsev, I. D., Grebel, E. K., \& Barsunova, O. Y. 2002, A\&A, 384, 72

Marlowe, A. T., Heckman, T. M., Wyse, R. F. G., \& Schommer, R. 1995, ApJ, 438, 563

Marlowe, A. T., Meurer, G. R., \& Heckman, T. M. 1999, ApJ, 522, 183

Marlowe, A. T., Meurer, G. R., Heckman, T. M., \& Schommer, R. 1997, ApJS, 112, 285

Masegosa, J., Moles, M., \& Campos-Aguilar, A. 1994, ApJ, 420, 576

Mas-Hesse, J. M., \& Kunth, D. 1999, A\&A, 349, 765

Méndez, D. I., \& Esteban, C. 1999, AJ, 118, 2723

Méndez, D. I., \& Esteban, C. 2000, A\&A, 359, 493

Meurer, G. R., Staveley-Smith, L., \& Killeen, N. E. B. 1998, MNRAS, 300, 705

Mirabel, I. F., \& Sanders, D. B. 1988, ApJ, 335, 104

Noeske, K. G. 1999, Diploma Thesis, University of Göttingen

Noeske, K. G., Guseva, N. G., Fricke, K. J., et al. 2000, A\&A, 361, 31

Noeske, K. G., Iglesias-Páramo, J., Vílchez, J. M., Papaderos, P., \& Fricke, K. J. 2001, A\&A, 371, 806

Noguchi, M. 2001, ApJ, 555, 289

Ott, J., Walter, F., Brinks, E., et al. 2001, AJ, 122, 3070

Pagel, B. E. J., Simonson, E. A., Terlevich, R. J., \& Edmunds, M. G. 1992, MNRAS, 255, 325

Papaderos, P., et al. 2003, in preparation

Papaderos, P. 1998, Ph.D. Thesis, University of Göttingen

Papaderos, P., \& Fricke, K. J. 1998, Highlights in X-ray Astronomy, ed. B. Aschenbach \& M. Freyberg

Papaderos, P., Loose, H.-H., Thuan, T. X., \& Fricke, K. J. 1996a, A\&AS, 120, 207 (P96a)

Papaderos, P., Loose, H.-H., Fricke, K. J., \& Thuan, T. X. 1996b, A\&A, 314, 59 (P96b)

Papaderos, P., Fricke, K. J., Thuan, T. X., Izotov, Y. I., \& Nicklas, H. 1999, A\&A, 352, L57 (P99)

Papaderos, P., Izotov, Y. I., Thuan, T. X., et al. 2002, A\&A, 393, 461

Pastoriza, M. G., \& Dottori, H. A. 1981, ApJ, 244, 27

Patterson, R. J., \& Thuan, T. X. 1996, ApJS, 107, 103

Peimbert, M., \& Torres-Peimbert, S. 1974, ApJ, 193, 327

Persson, S. E., Murphy, D. C., Krzeminski, W., Roth, M., \& Rieke, M. J. 1998, AJ, 116, 2475

Pustilnik, S. A., Kniazev, A. Y., Lipovetsky, V. A., \& Ugryumov, A. V. 2001, A\&A, 373, 24

Rieschick, A., \& Hensler, G. 2001, Ap\&SS Suppl., 277, 111

Sage, L. J., Salzer, J. J., Loose, H.-H., \& Henkel, C. 1992, A\&A, 265 , 19

Salzer, J. J., MacAlpine, G. M., \& Boroson, T. A. 1989, ApJS, 70, 479

Salzer, J. J., \& Norton, S. A. 1999, The Low Surface Brightness Universe, ASP Conf. Ser., 170, 253

Schaerer, D., Contini, T., \& Kunth, D. 1999, A\&A, 341, 399

Schlegel, D. J., Finkbeiner, D. P., \& Davis, M. 1998, ApJ, 500, 525

Schulte-Ladbeck, R. E., Hopp, U., Greggio, L., \& Crone, M. M. 2000, AJ, 120, 1713

Schulte-Ladbeck, R. E., Hopp, U., Greggio, L., Crone, M. M., \& Drozdovsky, I. O. 2001, AJ, 121, 3007

Schulz, J., Fritze - v. Alvensleben, U., Möller, C. S., \& Fricke, K. J. 2002, A\&A, 392, 1

Sérsic, J.-L. 1968, Atlas de Galaxias Australes, Observatorio Astronomico de Cordoba

Silk, J., Wyse, R. F. G., \& Shields, G. A. 1987, ApJ, 322, L59

Simpson, C. E., \& Gottesman, S. T. 2000, AJ, 120, 2975 
Smoker, J. V., Davies, R. D., Axon, D. J., \& Hummel, E. 2000, A\&A, 361,19

Stasinska, G., Comte, G., \& Vigroux, L. 1986, A\&A, 154, 352

Tammann, G. A., \& Sandage, A. 1985, ApJ, 294, 81

Taylor, C. L., Brinks, E., Grashuis, R. M., \& Skillman, E. D. 1995, ApJS, 99, 427

Telles, E., \& Terlevich, R. 1995, MNRAS, 275, 1

Telles, E., Melnick, J., \& Terlevich, R. 1997, MNRAS, 288, 78

Thuan, T. X. 1985, ApJ, 299, 881

Thuan, T. X. 1991, Massive Stars in Starbursts, ed. C. Leitherer, N. R. Walborn, T. M. Heckman, \& C. A. Norman

Thuan, T. X., Izotov, Y. I., \& Foltz, C. B. 1999, ApJ, 525, 105

Tully, R. B. 1988, Nearby Galaxies Catalog (Cambridge University Press)

Vacca, W. D., \& Conti, P. S. 1992, ApJ, 401, 543

Vallenari, A., \& Bomans, D. J. 1996, A\&A, 313, 713

van den Bergh, S. 2000, AJ, 119, 609 van Zee, L. 2000, AJ, 119, 2757

van Zee, L., Skillman, E. D., \& Salzer, J. J. 1998, AJ, 116, 1186 van Zee, L., Salzer, J. J., \& Skillman, E. D. 2001, AJ, 122, 121

Vanzi, L., Rieke, G. H., Martin, C. L., \& Shields, J. C. 1996, ApJ, 466, 150

Vanzi, L., Hunt, L. K., Thuan, T. X., \& Izotov, Y. I. 2000, A\&A, 363, 493

Vanzi, L., Hunt, L. K., \& Thuan, T. X. 2002, A\&A, 390, 481

Vennik, J., Hopp, U., \& Popescu, C. C. 2000, A\&AS, 142, 399

Vigroux, L., Souviron, J., Lachieze-Rey, M., \& Vader, J. P. 1988, A\&AS, 73, 1

Wainscoat, R. J., \& Cowie, L. L. 1992, AJ, 103, 332

Walter, F., Brinks, E., Duric, N., \& Klein, U. 1997, AJ, 113, 2031

Yasuda, N., Fukugita, M., \& Okamura, S. 1997, ApJS, 108, 417

Young, C. K., \& Currie, M. J. 1994, MNRAS, 268, L11

Young, C. K., \& Currie, M. J. 1995, MNRAS, 273, 1141 